\title{
Characterization of a PEDOT:PSS Electrolytic Device Using an in situ Spectroelectrochemical Technique
}

by

Eduardo Barrera Ramirez, B. Eng.

\author{
A thesis submitted to the \\ Faculty of Graduate and Postdoctoral Affairs \\ in partial fulfillment of \\ the requirements for the degree of
}

Master of Applied Science

in

Electrical and Computer Engineering

Ottawa-Carleton Institute for Electrical and Computer Engineering

Department of Electronics

Carleton University

Ottawa, Ontario, Canada

(C) 2013, Eduardo Barrera Ramirez 


\begin{abstract}
PEDOT:PSS, a conjugated polymer, has demonstrated great potential in emerging applications due to its variable conductivity and spectral attenuation. However, it is necessary to understand the relationship between these properties and the doping state of PEDOT:PSS. This work uses an in situ spectroelectrochemical experiment to measure conductance and spectral attenuation of PEDOT:PSS while being dedoped quasistatically with lithium ions. Results show a reversible decrease in conductivity during dedoping with a maximum on/off ratio of 2500 . The relationship between conductivity and doping state displays a sigmoidal shape with minimum conductivity at $\sim 1.2 \mathrm{mC}$ of intercalated charge. Migration speed of lithium through PEDOT:PSS was found to have a power-law speed-time relationship, where the exponent was on average 0.715 for applied voltages of $2 \mathrm{~V}-5 \mathrm{~V}$. This work provides important parameters to facilitate simulation of complex PEDOT:PSS polymer-electrolyte devices.
\end{abstract}




\section{Acknowledgements}

First and foremost, I would like to express my sincere gratitude to my advisor Dr. Steven P. McGarry for providing me with invaluable guidance and support that allowed me to complete this thesis. I would also like to thank him for his patience and fruitful advice and above all for being a great mentor.

I would like to thank Mr. Nagui Mikhail for providing me with the necessary equipment and tools to perform measurements. Finally, I also want to thank Dr. Luke O'Dell for his assistance during the NMR experiments performed at the National Research Council (NRC) of Canada.

I would like to thank my parents for always being concerned about my well-being and for their continuous support. I specially want to thank my beloved sister for her encouragement and advice during the many obstacles faced throughout this thesis. Her impeccable work ethic and determination during the completion of her own thesis was a constant reminder to always strive for excellence. I also want to thank my brother for being the best role model I have in my life and for all his helpful advice. 


\section{Table of Contents}

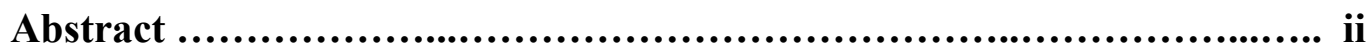

Acknowledgements .......................................................... iii

Table of Contents .............................................................. iv

List of Tables ................................................................. viii

List of Figures ................................................................... ix

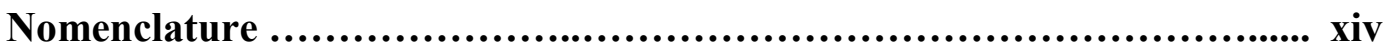

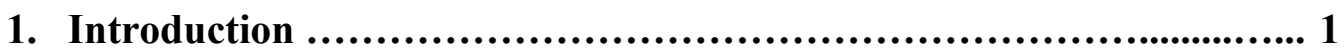

1.1. Thesis Objectives ................................................ 1

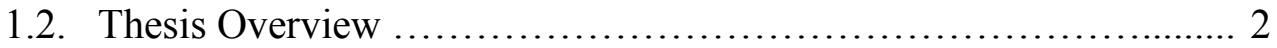

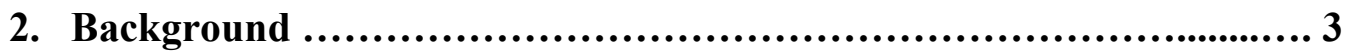

2.1. Introduction to Conjugated Polymers ............................... 3

2.1.1. Chemical Structure ............................................. 3

2.1.1.1. Molecular Bonds .......................................... 3

2.1.1.2. Bonding and Antibonding Orbitals .......................... 4

2.1.1.3. Sigma $(\sigma)$ and $\operatorname{Pi~}(\pi)$ Bonds ................................ 6

2.1.1.4. Orbital Hybridization in Saturated and Unsaturated

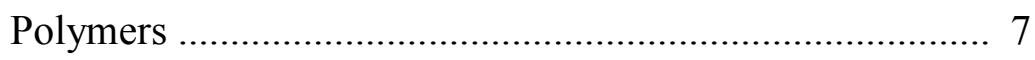

2.1.2. Energy Band Structure ............................................ 11

2.1.2.1. Metals, Semiconductors and Insulators ...................... 11

2.1.2.2. Conjugated Polymers ...................................... 12

2.1.2.3. Peierls Distortion ......................................... 16

2.1.3. Charge Carriers ............................................. 18

2.1.3.1. Solitons ..................................................... 18

2.1.3.2. Polarons .............................................. 21

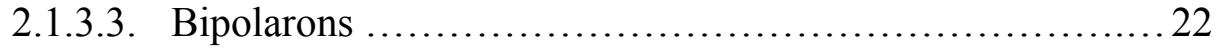

2.2. Electrochemical Systems ...................................... 24

2.2.1. Introduction ................................................... 24

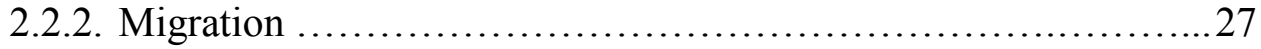




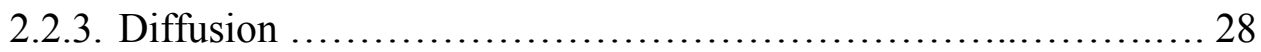

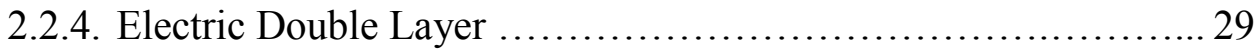

2.2.5. Solvation Effects …......................................... 30

2.3. Nuclear Magnetic Resonance (NMR) .............................. 32

2.3.1. Introduction .................................................... 32

2.3.2. Magnetic Field Interaction ...................................... 33

2.3.3. Net Magnetization Dynamics .......................................... 34

2.3.4. Pulsed Gradient Spin Echo NMR ................................ 38

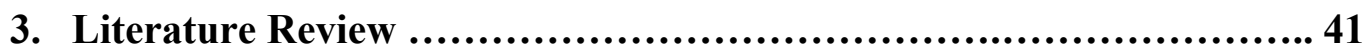

3.1. Material Properties of PEDOT:PSS ............................. 41

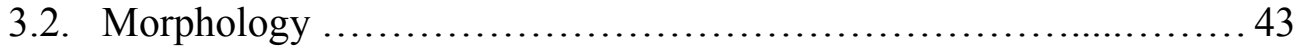

3.3. Charge Transport Model ...........................................45

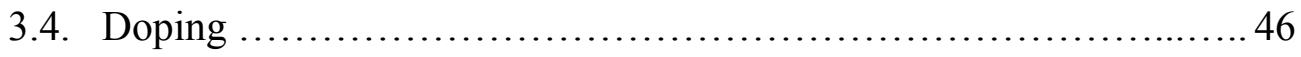

3.4.1. Electrochemical Doping ...................................... 48

3.5. Conductivity vs. Ion Concentration ............................... 50

3.6. Optical Properties .............................................. 51

4. Conductivity of PEDOT:PSS .........................................55

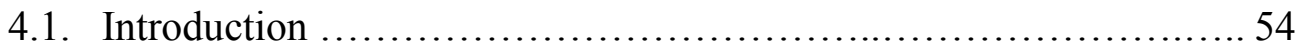

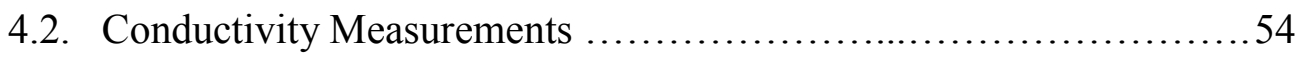

4.3. Conclusions ........................................................ 58

5. Lithium Diffusion: Pulsed Gradient Spin Echo NMR ..................... 59

5.1. Introduction .................................................... 59

5.2. Lithium Perchlorate Electrolyte Preparation .......................... 59

5.3. Experimental Setup and Results .................................6 60

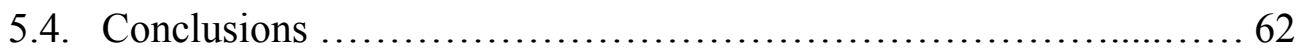

6. PEDOT:PSS Conductivity Dependence on Lithium Intercalation ........ 64

6.1. Introduction .................................................... 64

6.2. Device Geometry and Preparation ................................ 64

6.3. Calculation of Redox Sites Density ................................. 67

6.4. Spectral Attenuation Spectroscopy Setup ............................. 69

6.5. Electrochemical Setup ........................................ 72 
6.6. Spectroelectrochemical Experiment Control Program .............. 74

6.6.1. Type of Programs ...................................... 75

6.7. Experimental Results ...................................... 76

6.7.1. Preliminary Cycling .................................... 76

6.7.2. Resistive Measurements .................................. 77

6.7.3. Spectral Attenuation Measurements ........................ 85

6.8. Conclusions ............................................. 87

7. Video Analysis of Lithium Intercalation into PEDOT:PSS Films ...... 88

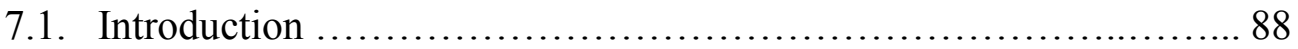

7.2. Device Geometry ........................................ 89

7.3. Experimental Setup and Explanation ............................ 90

7.4. Video Results Analysis .......................................... 92

7.4.1. Single Channel PEDOT:PSS Device ....................... 92

7.4.2. Notch PEDOT:PSS Device ................................. 96

7.4.2.1. Dedoping Videos ....................................... 96

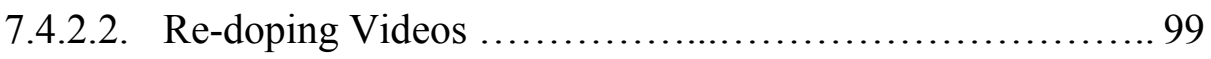

7.5. Conclusions .............................................. 102

8. Diode-like Behaviour of Asymmetric PEDOT:PSS Devices ............ 103

8.1. Introduction ............................................... 103

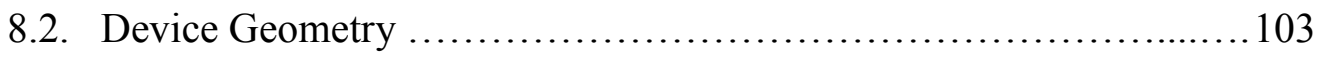

8.3. Experimental Setup and Explanation ........................... 105

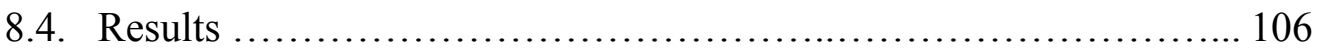

8.4.1. Voltage Amplitude Dependence ............................ 106

8.4.2. Frequency Dependence ..................................... 108

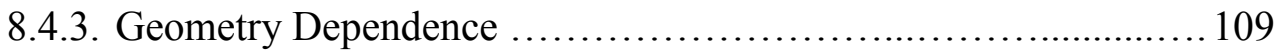

8.4.4. Self-Oscillatory Behaviour ................................ 111

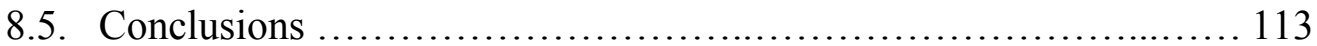

9. Thesis Conclusion .........................................................115

9.1. Summary .................................................. 115

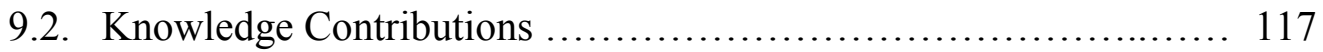

9.3. Improvements and Future Work ............................... 118 


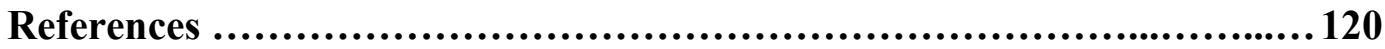




\section{List of Tables}

4.1. Conductivity measurements of PEDOT:PSS devices shown in Figure 4.1 ... 57

5.1. Experimental data for the diffusion coefficient of $\mathrm{Li}^{+}$ion for various electrolyte concentrations ...........................................6 61

8.1. Dimensions and initial resistance for each asymmetric PEDOT:PSS

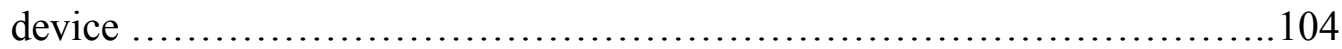

8.2. Width ratios for each asymmetric PEDOT:PSS device $\ldots \ldots \ldots \ldots \ldots \ldots \ldots \ldots . \ldots 104$ 


\section{List of Figures}

2.1. Constructive and destructive atomic orbital overlap .....................5 5

2.2. Antibonding and Bonding Molecular Orbitals ........................ 6

2.3. (a) $\sigma$ bonding between two $p_{z}$ orbitals and (b) $\pi$ bonding between two $p_{x}$ orbitals ..............................................6

2.4. Hybridization of and $s$ orbital with three $p$ orbitals $\left(p_{x}, p_{y}, p_{z}\right)$ into four $s p^{3}$ orbitals .................................................. 8

2.5. Chemical structure of polyethylene ................................ 9

2.6. Chemical structure of polyacetylene ................................ 10

2.7. (a) $s p^{2}$ hybridization and (b) $s p$ hybridization ............................. 11

2.8. A 1-D chain made from ions separated by a distance a used to model polyacetylene .................................................. 13

2.9. (a) Plot of energy vs. wave vector for a free electron and (b) for an electron in a periodic potential with periodicity a

2.10. (a) Undistorted 1-D chain model and (b \& c) two possible Peierls

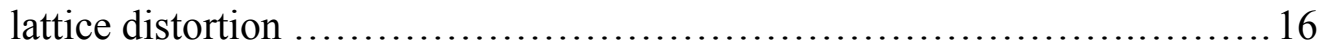

2.11. (a \&b) Two configurations for the Peierls distortion in polyacetylene

2.12. A kink in the bond alternation of a polyacetylene chain which creates a soliton and an unpaired electron pictured as a black dot

2.13. Spin and Charge relations for (a) a neutral soliton $[q=0, s=1 / 2]$, (b) anti-soliton $[q=0, s=-1 / 2]$, (c) negative soliton $[q=-e, s=0]$, and (d) positive soliton $[q=+e, s=0]$

2.14. (a) Formation of a negative polaron $[q=-e, s=1 / 2]$ due to the overlap of a soliton and negative soliton. (b) Formation of a positive polaron $[q=+e, s=1 / 2]$ due to the overlap of a soliton and positive soliton

2.15. (a) Combination of two polarons to make a bipolaron. (b) Overlap of bipolaron states into the formation of bipolaron energy bands

2.16. Illustration of a positive $[q=+e, s=0]$ and negative $[q=-e, s=0]$ bipolaron .24 
2.17. An isotropic or uniformly distributed ensemble of spin polarizations

2.18. An anisotropic ensemble of spin polarization constituting a net longitudinal magnetic moment .............................................. 36

2.19. Rotation of the net magnetic moment about the RF coil axis by 90 degrees... 37

2.20. Time diagram of a PGSE-NMR experiment using pulsed magnetic field gradients

3.1. Chemical structure of PEDOT:PSS made up of a PSS backbone (top) and a PEDOT chain (bottom) ionically bonded (with permission from [19])...... 42

3.2. (a) Chemical structure of PEDOT:PSS. (b) Individual PEDOT chains attached to long PSS chains. (c) Entangled PEDOT:PSS chains.

(d) Particles of entangled PEDOT:PSS chains (with permission from [19])

3.3. Lamellar structure of PEDOT:PSS illustrating the granular morphology made up of PEDOT-rich regions separated by PSS lamellas ............... 44

3.4. Illustration of an electrolytic cell and its components 48

3.5. Allowed optical transitions [shown by dotted arrows] in the case of (a) a soliton, (b) a polaron and (c) a bipolaron 52

4.1. Fabricated PEDOT:PSS devices used to measure the polymer conductivity...55

4.2. Profilometer scans for polymer devices with different widths: (a) $>1 \mathrm{~mm}$, (c) $0.7 \mathrm{~mm}$ and (b) $\leq 0.4 \mathrm{~mm}$ 56

4.3. Plot of conductivity values for every PEDOT:PSS device shown in Figure 4.1

5.1. Diffusion coefficient calculation using the Stejskal-Tanner curve fitting method

5.2. $\mathrm{Li}^{+}$ion diffusion coefficient and electrolyte conductivity as a function of concentration of $\mathrm{Li}^{+}$ions in a $\mathrm{LiClO} 4-\mathrm{PC}$ electrolyte.

6.1. PET device with wires attached by silver epoxy and before it was sealed ... 66

6.2. PET device after it was completely sealed 66

6.3. Diagram of the optical setup used in the spectroelectrochemical experiments 
6.4. Connections among the PET device, SMU, LCR meter and Scanner including the connections inside the $4 \times 5$ matrix switching card ....

6.5. Resistance and spectral attenuation at $625 \mathrm{~nm}$ for a PET device as a function of voltage during cycling

6.6. Resistance vs. applied voltage for a PET device during the spectroelectrochemical experiment

6.7. Resistance vs. applied voltage for a PET device during the reduction of the polymer film and for different maximum applied voltages

6.8. Resistance vs. applied voltage for a PET device during the oxidation of the polymer film

6.9. Resistance $v s$. Intercalated Charge for the polymer film in a PET device .... 83

6.10. Conductivity $v s$. Intercalated $\mathrm{Li}^{+}$ion concentration for the polymer film in a PET device in log scale (Linear scale is shown in the inset)

6.11. Illustration of total dry resistance $\left(2 R_{o}\right)$ and active resistance $\left(R_{A}\right)$ for the polymer film

6.12. Spectral Attenuation of the PEDOT:PSS film in a PET device during dedoping. The spectral attenuation (SA) represents the ratio of the signal blocked by the assembled polymer sample and the total signal measured without the polymer sample (Equation 6.2)

7.1. A single channel device. The black circle represents the area where the electrolyte was applied and the red dotted rectangle represents the region where the camera was focused

7.2. (left) A notch device and (right) a close up of the gap between the Short Region $(S R)$ and Long Region $(L R)$ of PEDOT:PSS

7.3. Setup for video analysis of $\mathrm{Li}^{+}$ion intercalation into PEDOT:PSS thin films

7.4. Displacement over time of the rising front for various applied voltages during the $1^{\text {st }}$ minute and (inset) during the entire 10 minutes

7.5. Logarithmic relationship between displacement and time during the first minute of dedoping. (Inset) $\alpha$ and $\beta$ values extracted by fitting experimental data to the power law 
7.6. Spatial and Temporal evolution of the rising front for an applied voltage of $5 \mathrm{~V}$ during 10 minutes

7.7. Spatial and Temporal evolution of the rising front for an applied voltage of $1.7 \mathrm{~V}$ during $20 \mathrm{~s}$

7.8. Spatial and temporal evolution of the rising front through a cross sectional path along the center of the polymer and for an applied voltage of $1.7 \mathrm{~V}$ during 20 s ......

7.9. Displacement of rising front over time along the center of the PEDOT:PSS film during dedoping at $1.7 \mathrm{~V}$ for all 5 videos. (Inset) Average ion speed during the initial $2 \mathrm{~s}$ of rising front motion for each video 99

7.10. Spatial and Temporal evolution of the falling front for an applied voltage of $-0.5 \mathrm{~V}$ during $20 \mathrm{~s}$ 100

7.11. Spatial and temporal evolution of the falling front through a cross sectional path along the center of the polymer and for an applied voltage of $-0.5 \mathrm{~V}$ during 20s

7.12. Spatial and Temporal evolution of the relative ion density for the re-doping of the $L R$ polymer without an applied voltage and during 90s .............. 102

8.1. Asymmetric PEDOT:PSS devices with varying width ratios .................. 104

8.2. Circuit diagram used to test the asymmetric PEDOT:PSS devices .......... 105

8.3. Voltage dependence on the I-V curve of a symmetric device at $100 \mathrm{mHz}$..... 107

8.4. Voltage dependence on the I-V curve of a symmetric device at $1.0 \mathrm{~Hz} \ldots \ldots . .108$

8.5. Frequency dependence on the I-V curve for a symmetric device for a source voltage of $7 \mathrm{~V}$ 109

8.6. Electric potential distribution for (a) a symmetric PEDOT:PSS device [APD-1] and (b) asymmetric PEDOT:PSS device [APD-5]. Colour bar is given in units of volts

8.7. Electric Field through cross sectional path for a symmetric PEDOT:PSS device [APD-1] and an asymmetric PEDOT:PSS device [APD-5]

8.8. I-V curves for 4 asymmetry and 1 symmetric device for a source range of $\pm 1.5 \mathrm{~V}$ and a frequency of $40 \mathrm{mHz}$ 
8.9. (left) Anomalous oscillatory behaviour in the electrical response of APD-3 at $100 \mathrm{mHz}$. (right) close up of oscillations for $8 \mathrm{~V}$ and $100 \mathrm{mHz}$............. 112 


\section{Nomenclature}

\begin{tabular}{|c|c|}
\hline$k_{B}$ & Boltzmann constant $[\mathrm{J} / \mathrm{K}]$ \\
\hline$N_{i}$ & Ion flux or flow rate per unit area $\left[\mathrm{mol} /\left(\mathrm{m}^{2} \cdot \mathrm{s}\right)\right]$ \\
\hline$D_{i}$ & Diffusion coefficient $\left[\mathrm{m}^{2} / \mathrm{s}\right]$ \\
\hline$c_{i}$ & Concentration $\left[\mathrm{mol} / \mathrm{m}^{3}\right]$ \\
\hline$\mu_{i}$ & Mobility $\left[\mathrm{m}^{2} /(\mathrm{V} \cdot \mathrm{s})\right]$ \\
\hline$V$ & Electric potential $[\mathrm{V}]$ \\
\hline$J$ & Current density $\left[\mathrm{A} / \mathrm{m}^{2}\right]$ \\
\hline$F$ & Faraday's constant $[\mathrm{C} / \mathrm{mol}]$ \\
\hline$Z$ & Charge number [unitless] \\
\hline$\varepsilon_{r}$ & Dielectric constant [unitless] \\
\hline$\varepsilon_{o}$ & Permittivity of free space $[\mathrm{F} / \mathrm{m}]$ \\
\hline$\sigma$ & Conductivity $[\mathrm{S} / \mathrm{m}]$ \\
\hline$\sigma$ & Sigma-bond symbol \\
\hline$q$ & Charge $[\mathrm{C}]$ \\
\hline$e$ & Elementary charge constant $[\mathrm{C}]$ \\
\hline PEDOT: PSS & Poly(3,4-ethylenedioxythiophene):Poly(4-styrenesulfonate) \\
\hline PEDOT & Poly(3,4-ethylenedioxythiophene) \\
\hline$P S S A$ & Poly(styrene sulfonic acid) \\
\hline EDOT & Ethylenedioxythiophene monomer \\
\hline$V R H$ & Variable range hopping \\
\hline$P G S E$ & Pulsed gradient spin echo \\
\hline HOMO & Highest occupied molecular orbital \\
\hline
\end{tabular}


Lowest unoccupied molecular orbital

$N M R$

Nuclear magnetic resonance

$N_{A}$

Avogadros number $\left[\mathrm{mol}^{-1}\right]$ 


\section{Chapter 1}

\section{Introduction}

\subsection{Thesis Objectives}

The primary objective of this work was to characterize a poly $(3,4-$ ethylenedioxythiophene)/poly(styrenesulfonate) (PEDOT:PSS) polymer thin film by determining the relationship between the conductivity of the polymer and the concentration of intercalated counter ions in the polymer.

This was accomplished using spectroelectrochemical experiments in which the conductivity and spectral attenuation of the polymer were monitored while Lithium $\left(\mathrm{Li}^{+}\right)$ ions were intercalated. The ion migration through the polymer was studied by means of a video analysis that quantified the changes in colour due to ion intercalation. The electrolyte was characterized by determining the relationship between the diffusion coefficient of $\mathrm{Li}^{+}$ions and the concentration of these ions in the electrolyte. The results from this thesis are necessary to develop a simulation of the dynamics of a PEDOT:PSS electrolytic device and to investigate complex device structures before they are fabricated and tested in the laboratory.

As a test case, a simple polymer-electrolyte structure was fabricated and its electrical response measured to determine the voltage, frequency and geometry dependence of its electrical response. The results obtained for this polymer-electrolyte structure can be compared with future simulation of these devices in order to validate the accuracy of the simulation. 


\subsection{Thesis Overview}

The following two chapters present the necessary background knowledge for the work described in this thesis. Chapter 2 starts with an introduction to polymers and the basic structure of conjugated polymers (CP), which discusses the electrical properties of $\mathrm{CP}$ and the nature of charge carriers in $\mathrm{CP}$. A basic introduction to mass transport dynamics in electrochemistry is also provided. Finally, the principles of Nuclear Magnetic Resonance (NMR) are outlined along with the description of a special technique used in this work called Pulse Gradient Spin Echo (PGSE) NMR. Chapter 3 covers specific information regarding the conducting polymer PEDOT:PSS used in this work.

The remainder of the thesis covers the experiments, results, analysis and conclusions of the work. Chapter 4 describes how the conductivity of PEDOT:PSS was measured and provides the experimental results. In addition, chapter 5 describes the NMR methods used to determine the relationship between $\mathrm{Li}^{+}$ion diffusion coefficient and $\mathrm{Li}^{+}$ion concentration in the electrolytes. Chapter 6 covers the setup of the spectroelectrochemical experiment and discusses the experimental results leading to the relationship between polymer conductivity and intercalated ion concentration. Chapter 7 describes the video analysis of ion migration into the polymer. It also provides the data analysis and the qualitative description of the ion migration dynamics. Chapter 8 presents a detailed description of the fabrication of an asymmetric polymer-electrolyte structure and the results obtained for its electrical response. Finally, Chapter 9 concludes this thesis by summarizing the experimental results and providing suggestions for improvements. 


\section{Chapter 2}

\section{Background}

\subsection{Introduction to Conjugated Polymers}

Polymers are considered to be primarily insulating material, in contrast to inorganic solids which can behave as semiconductors, metals or insulators. However, this notion changed due to the discovery of semiconductor properties in polymers after 1950 . Furthermore, a discovery in the 1970 s showed that polymers could reach conductivities of metallic level and this ignited an immense research interest that led to the development of various conductive polymers. Although, the properties of inorganic materials and conductive polymers resemble each other, their microscopic behaviours are fundamentally different due to the differences between the rigid crystal lattices of inorganic materials and the deformable molecular structure of polymers. For instance, local deformation in the structure of a material caused by the presence of charge carriers is significantly larger in conductive polymers than in inorganic semiconductors [1].

\subsubsection{Chemical Structure}

\subsubsection{Molecular Bonds}

The family of polymers used in this work are considered organic compound made of chain-like molecules. These chains are composed of repeated molecular units that are linked by covalent bonds. The backbone of these organic polymers is primarily carbon atoms in combination with oxygen and/or nitrogen atoms. Also, the covalent bond 
between the two carbon atoms is the result of sharing of electrons [1]. In order to understand how conjugated polymers obtain their conducting properties one must first consider the way molecular orbitals are created from atomic orbitals as atoms come together to form a molecule. In the case of an atom, by definition, the atomic orbital describes the probability of finding an electron in a given volume at some distance from the nucleus. The simplest case is the hydrogen atom, where the system is made up of an electron and a proton. In this case, the wavefunction of the electron in the ground state is called the $1 \mathrm{~s}$ orbital and it is spherically symmetric [2].

Atomic orbitals are labeled according to the principal $(n)$, azimuthal $(l)$ and magnetic $\left(m_{l}\right)$ quantum numbers. The principal quantum number determines the energy level and the azimuthal quantum number determines the quantized orbital angular momentum of the electron within the orbital. The azimuthal quantum number can have a value of 0 to $n$ 1. In the case of the ground state of hydrogen, the label of $1 s$ refers to a principal quantum number of 1 and the $s$ represents the orbital angular momentum, which in this case is zero. For historical reasons, the orbital angular momentum receives letter labels $(s=0, p=1, d=2, f=3 \ldots)$. The magnetic quantum number describes the projection of the angular momentum along an arbitrary axis that can range from $-l$ to $l$. The quantized spin of an electron $\left(m_{s}\right)$ can either be $1 / 2$ or $-1 / 2$. All of these quantum numbers describe the state of an electron within an atomic orbital [2].

\subsubsection{Bonding and Antibonding Orbitals}

When two hydrogen atoms bond to form a hydrogen molecule $\left(\mathrm{H}_{2}\right)$, the wavefunctions of the two electrons overlap each other and the result is two molecular 
orbitals. These orbitals are called a bonding orbital and an antibonding orbital, which are caused by constructive and destructive interference, respectively (Figure 2.1) [1].

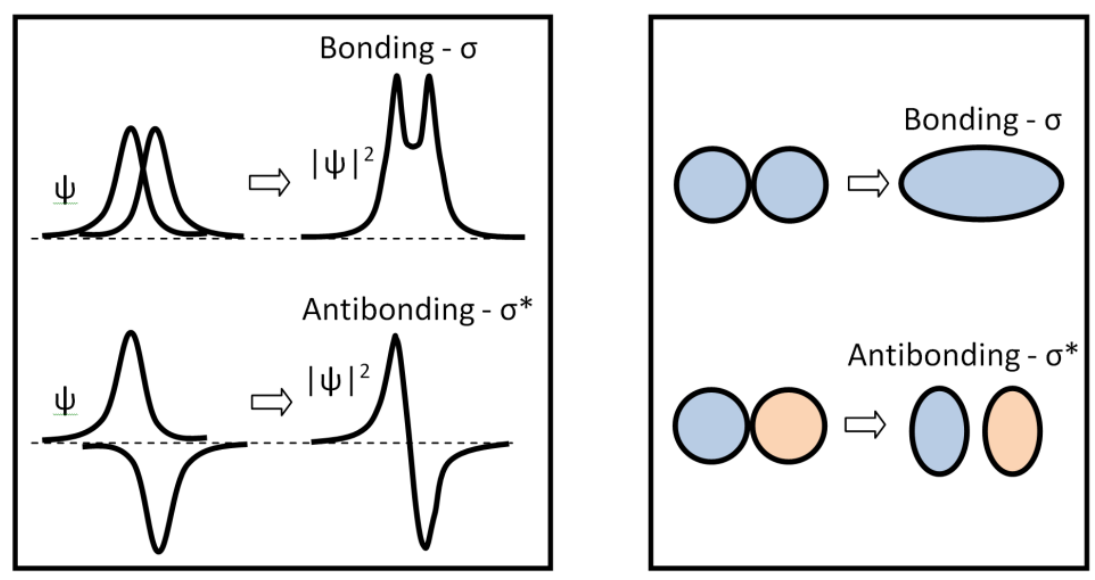

Figure 2.1 Constructive and destructive atomic orbital overlap

In the case of the bonding orbital, the wavefunctions constructively interfere to create a higher probability density of electrons between the two protons, which decreases the electrostatic repulsion between the two protons. Therefore an electron in the bonding orbital would have lower energy than an electron in the atomic orbital of a single hydrogen atom. The opposite occurs in the case of an antibonding orbital since there is a smaller probability density of electrons between the two protons due to destructive interference between the two wavefunctions. Therefore, there is no screening of the electrostatic repulsion between the two protons, which causes the electrons in the antibonding orbital to have higher energy than electrons in the atomic orbitals [1]. The energy difference between the antibonding molecular orbital and the atomic orbital is the same as the energy difference between the atomic orbital and the bonding molecular orbital (Figure 2.2). 


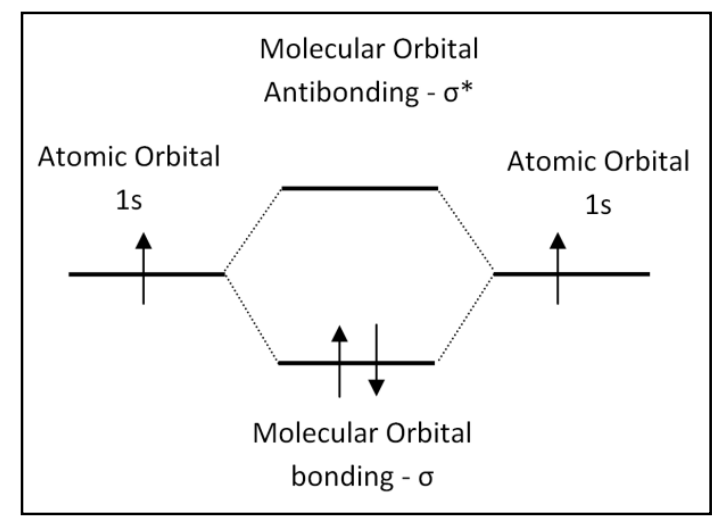

Figure 2.2 Antibonding and Bonding Molecular Orbitals

\subsubsection{Sigma $(\sigma)$ and $\mathrm{Pi}(\pi)$ Bonds}

Whenever the bonding and antibonding orbitals do not have a nodal plane along the internuclear axis, then these orbitals result from a sigma $(\sigma)$ bond and hence receive the name of $\sigma$ and $\sigma^{*}$ orbitals respectively (the asterisk simply denotes the antibonding orbital). The internuclear axis represents an imaginary straight line that crosses both nuclei in a bond. On the other hand, bonding and antibonding orbitals that have a nodal plane along the internuclear axis are formed by $\mathrm{Pi}(\pi)$ bonding. These bonding and antibonding orbitals receive the name of $\pi$ or $\pi^{*}$ orbital, respectively, and the atoms are not free to rotate along the bond. A $\sigma$-bond is stronger than $\pi$-bond due to a larger overlap of the atomic orbitals. Figure 2.3 (a) and (b) illustrate how two $p$ orbitals can form a $\sigma$ bond or a $\pi$ bond.

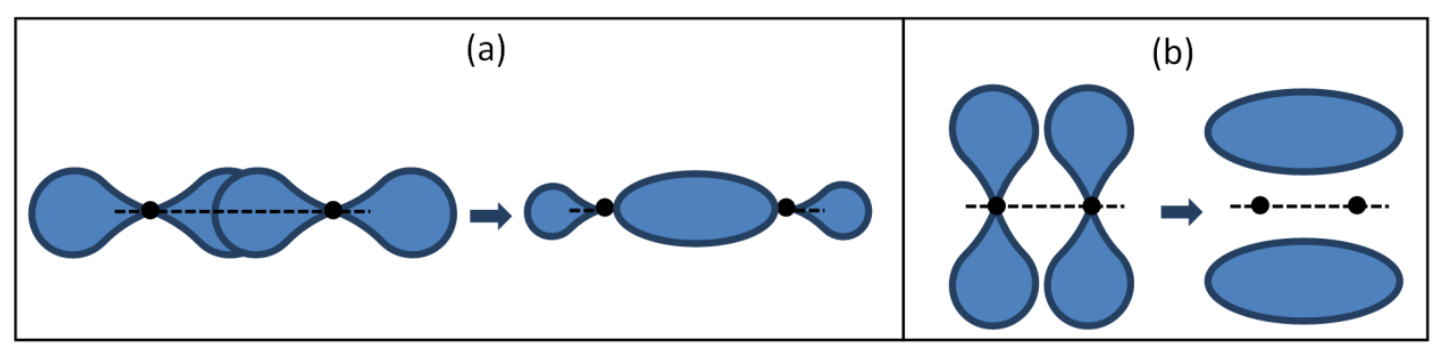

Figure 2.3 (a) $\sigma$ bonding between two $p_{z}$ orbitals and (b) $\pi$ bonding between two $p_{x}$ orbitals 
Unlike $s$ orbitals, $p$ orbitals have 3 orientations $\left(p_{x}, p_{y}, p_{z}\right)$ that are perpendicular to each other and are related to the magnetic quantum number $\left(m_{l}\right)$. If one arbitrarily assigns the internuclear axis to be along the $z$ axis, then the overlap between two $p_{z}$ orbitals will result in $\sigma_{p z}$ and $\sigma_{p z}^{*}$ molecular bonds (Figure 2.3a). On the other hand, the overlap between two $p_{x}$ or two $p_{y}$ orbitals would produce molecular orbitals that have nodes along the internuclear axis. Therefore, these bonds would be called $\pi_{p x}$ and $\pi_{p x}^{*}$ or $\pi_{p y}$ and $\pi_{p y}^{*}$ orbitals respectively (Figure $2.3 \mathrm{~b}$ ). Every time a molecular bond is formed between two atoms there will be bonding and antibonding, but for brevity the molecular bond will be referred as a $\sigma$ or $\pi$ bond without making explicit reference to the antibonding $\left(\sigma^{*}\right.$ or $\left.\pi^{*}\right)$ bond.

\subsubsection{Orbital Hybridization in Saturated and Unsaturated Polymers}

Conducting organic polymers have a backbone of linear carbon chains which can form two hybrid structures in order to lower the total energy and have spatially directed chemical bonds. These structures are called tetrahedral and hexagonal geometry. Tetrahedral geometry refers to nonconducting materials such as polyethylene or saturated polymers, while hexagonal geometry refers to conducting materials including graphite and conjugated or unsaturated polymers. In order to explain how these hybrid structures are formed one must understand the process of atomic orbital hybridization [3].

In some cases the $p$ and $s$ orbitals of an atom mix and create hybrid orbitals that can overlap with orbitals from another atom to form molecular orbitals. This mixing process is called hybridization of atomic orbitals and can occur by means of electron promotion if it is energy favourable. In the case of polyethylene, the 4 valence electrons of a carbon 
atom that are found in the $2 s$ and $2 p$ orbitals will be rearranged into 4 hybrid orbitals and therefore each holds a single unpaired electron. This is done by promoting an electron from the $2 s$ orbital into the $2 p_{z}$ orbital, which results in one unpaired electron in the $2 s$ orbital and in the $2 p_{z}$ orbital. Since the 4 unpaired electrons are in different orbitals, namely one in a $2 s$ orbital and three in the $2 p$ orbitals, these four orbitals will mix into linear combinations to form four new hybrid orbitals called $2 s p^{3}$ orbitals. These hybrid orbitals have the same shape and energy (since the principal quantum number is 2) but have different orientations and form a regular tetrahedron structure shown in Figure 2.4.

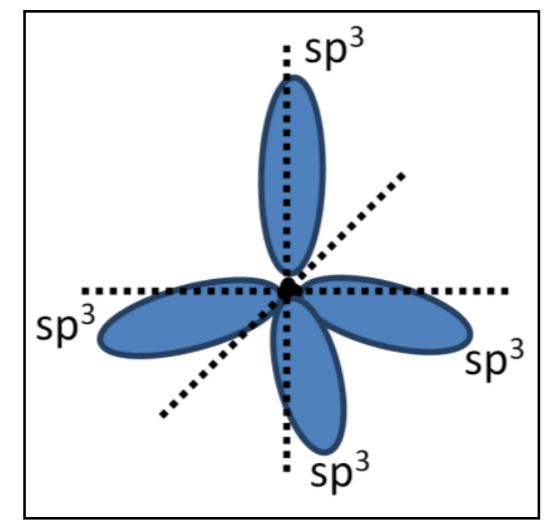

Figure 2.4 Hybridization of and $s$ orbital with three $p$ orbitals $\left(p_{x}, p_{y}, p_{z}\right)$ into four $s p^{3}$ orbitals

A hydrogen atom can now bond to two of the four $2 s p^{3}$ orbitals to form a $\sigma$ covalent molecular bond and the remaining $2 s p^{3}$ orbitals to form a $\sigma$ covalent molecular bond with two neighbouring carbon atoms. Polymers with a carbon backbone that has $\sigma$ bonds made from $s p^{3}$ hybrid orbital overlaps are called saturated polymers. Saturated polymers, such as polyethylene (Figure 2.5), are insulators because each valence electron from a carbon atom is bound to a covalent bond and hence there are no available electrons to conduct current [3] [4]. 


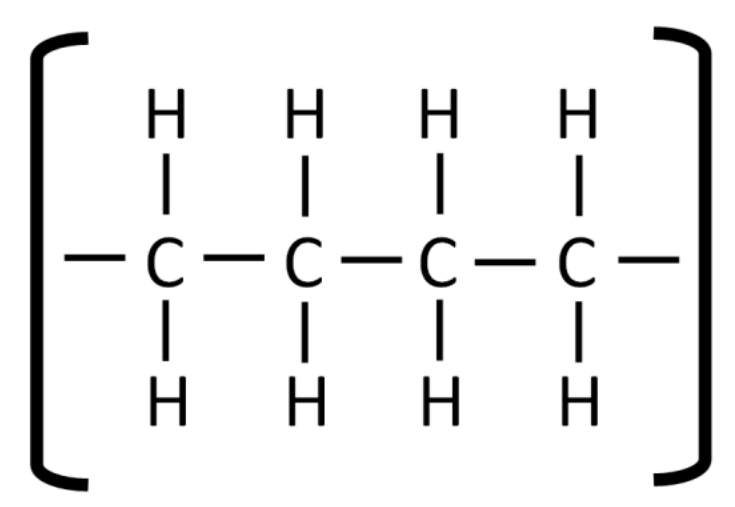

Figure 2.5 Chemical structure of polyethylene

In polyacetylene, the simplest conducting polymer, there is also a $2 s$ electron promoted to the empty $2 p_{z}$ orbital, but only two $p$ orbitals and the $s$ orbital are hybridized into $2 s p^{2}$ orbitals. Consequently, the carbon atom has three $2 s p^{2}$ and one $2 p$ orbital. Each carbon atom will still bond with its two neighbouring carbon atoms by overlapping one of their $s p^{2}$ together and this overlapping will create $\sigma$ molecular bonds. The remaining $s p^{2}$ orbitals in each carbon atom will form additional $\sigma$ molecular bonds with a hydrogen atom. The unpaired electron in the $2 p$ orbital of a carbon atom is called a $\pi$ electron and can form a $\pi$ bond with the other unpaired electron of any adjacent carbon atom. This $\pi$ bond is parallel to the $\sigma$ bond between the carbon atoms and therefore these carbon atoms form a double bond. Polymers that have this type of $s p^{2}$ hybridization are called unsaturated. A chain of carbon atoms that have this type of $\pi$ bonding is referred to as a conjugated $\pi$ system (Figure 2.6) [3] [4]. 


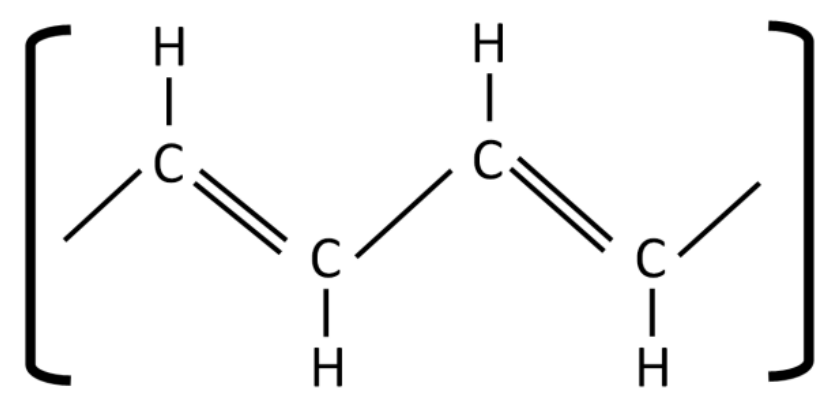

Figure 2.6 Chemical structure of polyacetylene

The other type of bond that can occur between carbon atoms is a triple bond made up of one $\sigma$ bond and two $\pi$ bonds. In this case, the hybridization occurs between the $2 s$ orbital and one $2 p$ orbital, which creates two $2 s p$ hybrid orbitals. This leaves two $2 p$ orbitals with an unpaired electron in each orbital. Therefore, a $\sigma$-bond is formed between the $2 s p$ orbital of each carbon atom and the remaining $2 s p$ orbital in each atom bonds with a hydrogen atom. Also, each $2 p$ orbital from one carbon atom forms a $\pi$ bond with the adjacent carbon atom. Since there are two $2 p$ orbital in each carbon atom, there will be two $\pi$ bonds. The two $\pi$ bonds and the $\sigma$ bond between two carbon atoms constitute a triple bond (Figure 2.7(b)). However, a carbon backbone in a polymer cannot be made from $2 s p$ hybridization because each carbon atom needs to have a $\sigma$ bond with two other carbon bonds in order to form a linear carbon chain. Hence, conjugated polymers are made from $2 s p^{2}$ hybridization [1] [4]. Figure 2.7 (a) and (b) show the $2 s p^{2}$ and $2 s p$ hybridization configurations, respectively. 


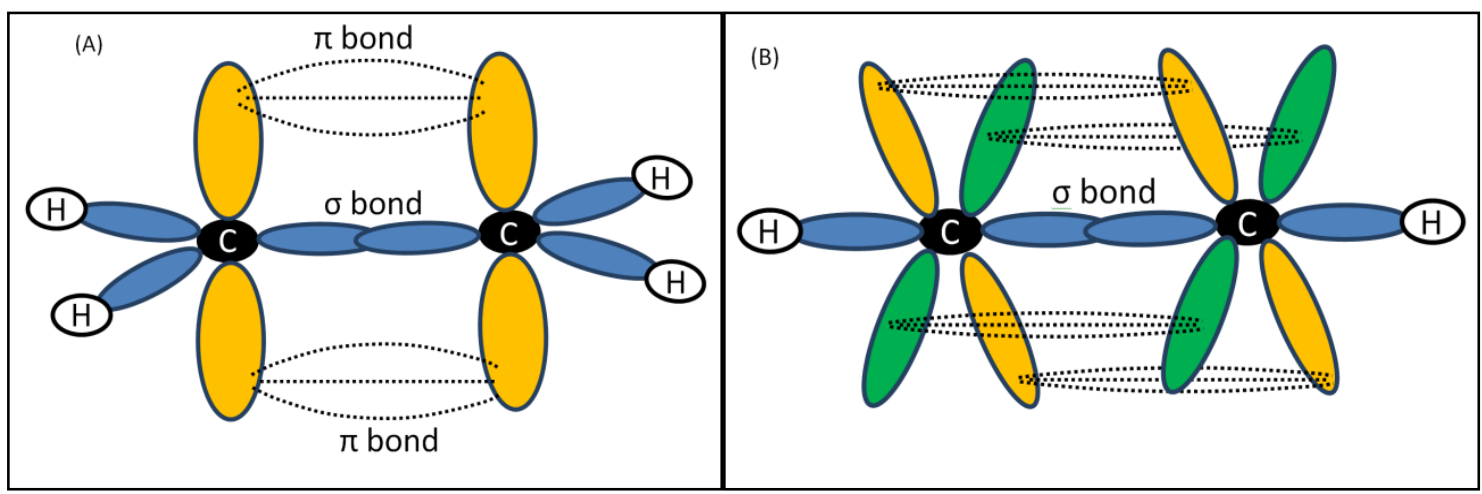

Figure 2.7 (a) $s p^{2}$ hybridization and (b) $s p$ hybridization

It is worth noting that unsaturated polymers are less chemically stable than saturated polymers because electrons found within a $\sigma$ bond are more difficult to remove than electrons found in $\pi$-bonds. However, unsaturated polymers have semiconducting and even metallic properties unlike saturated polymers [1] [4].

\subsubsection{Energy Band Structure}

\subsubsection{Metals, Semiconductors and Insulators}

In the discussion of molecular orbitals, only two atoms were considered in the formation of a molecule and its resulting molecular orbitals. In this case the atomic orbitals of both atoms are degenerate in energy, but as the two atoms come together each set of degenerate atomic orbitals splits into two nondegenerate molecular orbitals (different energy). In the general case of $N$ atoms, each atomic orbital splits into $N$ nondegenerate energy levels which are evenly spaced. However, as $N$ becomes very large the spacing among the $N$ energy levels becomes so insignificant that it leads to the formation of continuous energy bands [5]. 
Neighbouring energy bands can overlap or be separated by an energy gap called a bandgap and each energy band has a specific electron occupancy that depends on the temperature. In the case of a metal, there is a partially filled energy band that allows electrons to move to adjacent unoccupied levels in response to an applied electric field. On the other hand, an insulating material has a bandgap of several eV separating a filled energy band and an unfilled energy band; therefore electrons cannot be thermally excited unto the unfilled band in order for them to become mobile charge carriers. The unfilled energy band and filled energy band are called the conduction and valence band, respectively. A semiconductor is similar to an insulator, however its energy bandgap is low enough that electrons can more easily be thermally excited from the valence band to the conduction band [6]. Consequently, a temperature rise in a semiconductor promotes more charge carriers onto the conduction band and hence the conductivity increases. Meanwhile, as temperature increases the conductivity of a metal decreases because electrons are more likely to collide with the immobile atom lattice and charge transport becomes inefficient [7].

\subsubsection{Conjugated Polymers}

In the case of conjugated polymers, the $\sigma$ bonding and $\pi$ bonding are the two factors that will determine the energy band structure and hence the electronic properties of the polymer. The first factor is the $\sigma$ bonding between the $s p^{2}$ hybrid orbitals of adjacent carbon atoms that is responsible for the strength of the polymer. In the case of a polymer made from many carbon atoms these $\sigma$ and $\sigma^{*}$ energy levels create a $\sigma$-band and a $\sigma^{*}$ band, respectively, that are separated by an energy bandgap. Any electron excitation from 
the $\sigma$-band to the $\sigma^{*}$-band will lead to degradation of the polymer because the polymer is held together by the electrons in the $\sigma$-band, hence these electron excitation have no interesting electronic properties [3].

The origin of the energy bands due to $\pi$ bonding will be explained by using polyacetylene as an example. If one models polyacetylene as a simple 1-D chain made of $N$ positive ion cores (Figure 2.8) evenly separated by a distance a, then the length $(L)$ of the chain would be approximately equal to $N$ times a and an electron within this 1D chain would experience a periodic potential [1].

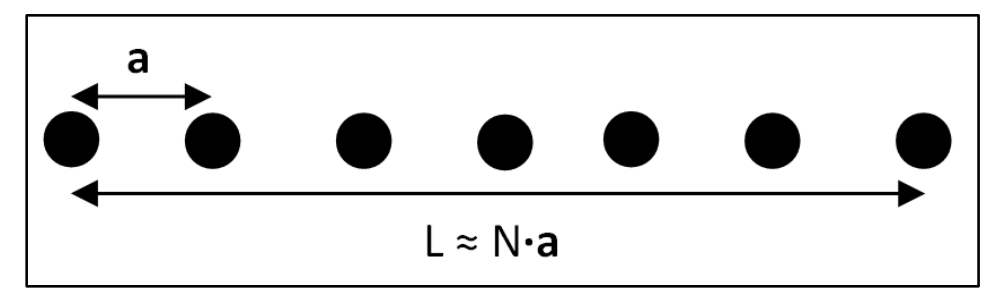

Figure 2.8 A 1-D chain made from ions separated by a distance a used to model polyacetylene

The wavefunction of the electron travelling through the periodic potential will undergo Bragg reflections, similarly to the reflections of X-rays used in X-ray crystallography. The Bragg condition (equation 2.1) is satisfied when the wave vector of the incident electron equals half of the reciprocal lattice vector $(G)[6]$.

$$
\begin{gathered}
G=\frac{2 \pi n}{a} \\
k_{B}=\frac{G}{2}=\frac{ \pm n \pi}{a}
\end{gathered}
$$

When the wave vector is equal to the value shown in equation 2.1, then the incident and reflected waves can set up a standing wave with either a crest centered at the ion 
cores or in between them. The modulus of each of these two standing waves represents the probability density for the electron. Therefore, the standing wave that corresponds to an electron distribution that is concentrated near the ion cores would have an average potential energy higher than the other standing wave, which corresponds to an electron distribution that is concentrated between the ion cores. This difference in energies for the same wave vector is the origin of the energy bandgap. If one considers the first energy gap $(n=1)$, there would be a continuous band for $k$-values from 0 up to $\pm \pi /$ a (Figure 2.9) [6].
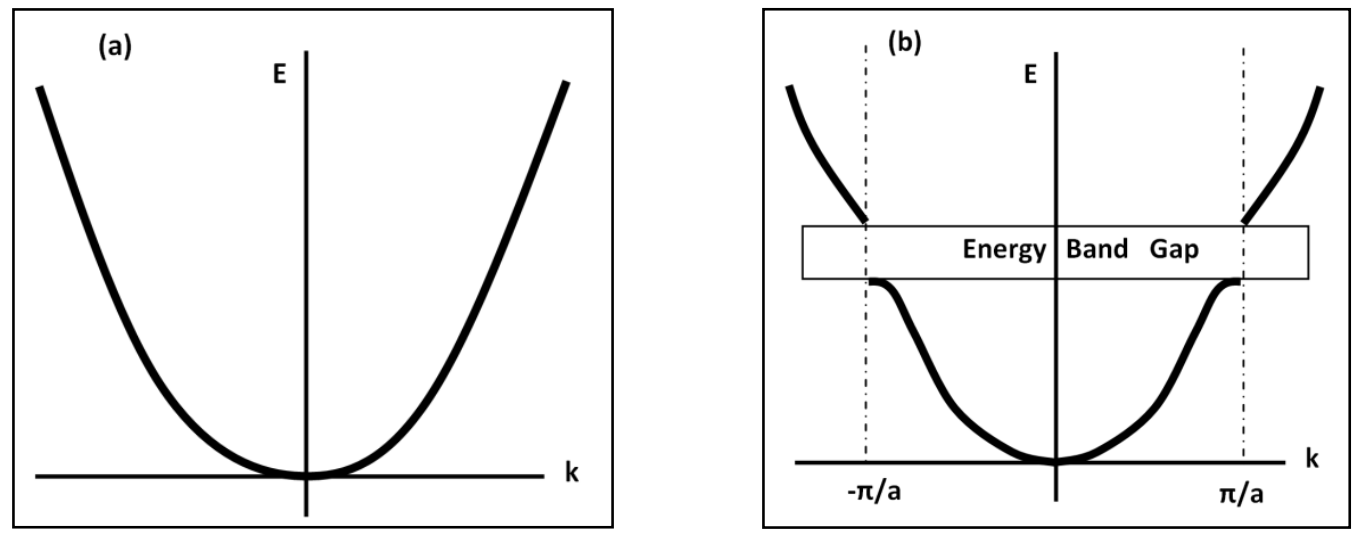

Figure 2.9 (a) Plot of energy vs. wave vector for a free electron and (b) for an electron in a periodic potential with periodicity a

Using Bloch's theorem, a possible solution to the Schrodinger equation in the case of the periodic potential would be given by equation 2.2 , where $u_{k}(x)$ is a cyclic function with the same period as the potential, $k$ is the wave vector and $L$ is the chain length [1].

$$
\psi(x)=e^{ \pm i k x} u_{k}(x) \quad \text { where } u_{k}(x)=u_{k}(x+L)
$$

The periodic condition from the Bloch's wavefunction can be used to derive an expression for the spacing between $k$-states given by equation 2.3 . 


$$
\begin{gathered}
\psi(x)=\psi(x+L) \\
e^{i k x} u_{k}(x)=e^{i(x+L) k} u_{k}(x+L) \\
1=e^{i 2 \pi n}=e^{i k L} \\
2 \pi n=k L \\
k_{n}=\frac{2 \pi n}{L} \\
\Delta k=\frac{2 \pi}{L}
\end{gathered}
$$

Since the maximum occupied energy level by an electron is the Fermi level at absolute zero, then the Fermi momentum $\left(k_{F}\right)$ would represent the highest $k$-state occupied by an electron [2]. The number of $k$-states up to $k_{F}$ is equal to the Fermi momentum divided by the spacing between $k$-states $(\Delta k)$. However the electron can have positive or negative wavenumbers, and therefore the number of $k$-states up to $-k_{F}$ must also be included which increases the total $k$-states $\left(\mathcal{N}_{k_{F}}\right)$ by a factor of two. According to the Pauli Exclusion Principle, each $k$-state can hold up to 2 electrons, hence $\mathcal{N}_{k_{F}}$ is equal to the total number of electrons divided by two. In polyacetylene, each carbon contributes one $\pi$ electron, which means that there are $N$ electrons in the 1-D chain since there are $N$ carbon atoms. Also, $\mathcal{N}_{k_{F}}$ would be equal to $N / 2$. Therefore, in this case the Fermi momentum is equal to half of the Bragg condition as shown in equation 2.4, which means the valence band is half filled and polyacetylene is a 1-D metal. This means that undistorted polyacetylene should be a 1-D metal even though it is actually a 
semiconductor, as revealed by experiment [1]. This discrepancy can be resolved by the Peierls distortion which occurs in polyacetylene.

$$
\begin{gathered}
\mathcal{N}_{k_{F}}=2\left(\frac{k_{F}}{\Delta k}\right)=\frac{k_{F} L}{\pi}=\frac{N}{2} \\
k_{F}=\frac{N}{2} \frac{\pi}{L}=\frac{\pi}{2 a}
\end{gathered}
$$

\subsubsection{Peierls Distortion}

In 1955, Peierls proved that a lattice distortion, called Peierls distortion, always appears within any 1-D metal because the distortion lowers the electronic energy of the lattice by an amount that is greater than the energy required to produce the lattice distortion, which makes the distortion process more energy favourable [1]. If the distortion within the 1-D chain model occurs as shown in Figure 2.10, then the previous ion core is now represented by two ion cores. This means that the chain would now have N/2 effective ion cores, the effective ion core separation would be $2 \mathbf{a}$ and the length of the chain would still be approximately $N \mathbf{a}$.

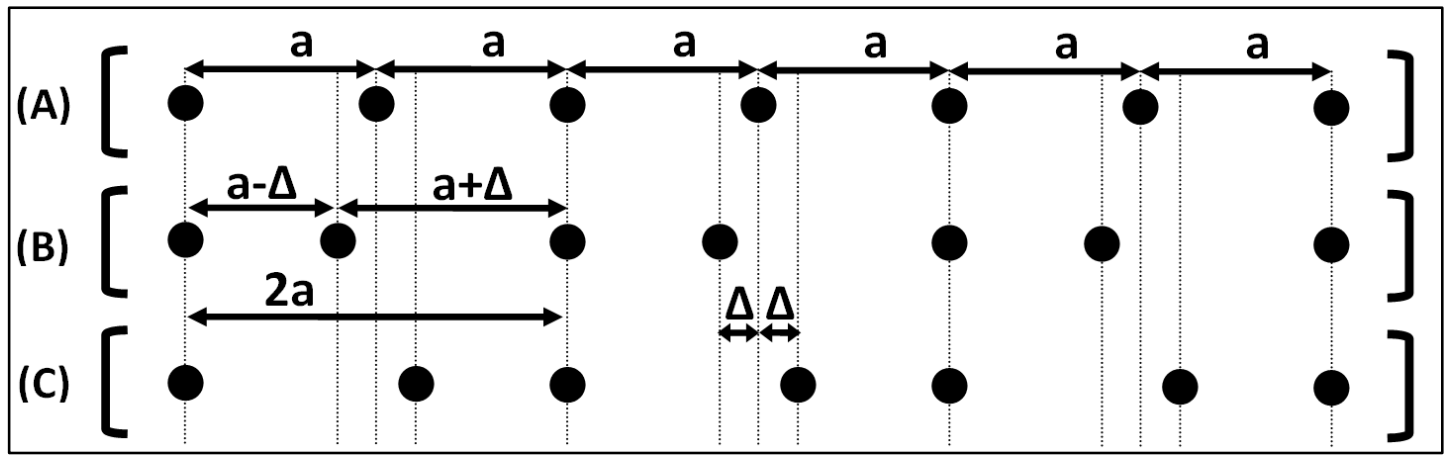

Figure 2.10 (a) Undistorted 1-D chain model and (b \& c) two possible Peierls lattice distortion 
In equation 2.4, $N$ and the length of the chain remain the same after the Peierls distortion, hence $k_{f}$ does not change. However, equation 2.1 shows that the Bragg condition depends directly on the ion core separation, and therefore the Bragg condition decreases by a factor of a half (equation 2.5).

$$
k_{B}=\frac{ \pm n \pi}{a^{\prime}}=\frac{ \pm n \pi}{2 a}
$$

The comparison between equation 2.4 and 2.5 shows that in $k$-space the bandgap and the Fermi momentum occur at the same $k$-value. As a result, the previously half-filled continuous band splits into a filled valance band (Highest Occupied Molecular Orbital (HOMO) band) and an empty conduction band (Lowest Unoccupied Molecular Orbital (LUMO) band) [1]. Therefore, polyacetylene becomes a semiconductor which agrees with the experimental measurement of its bandgap of $1.4 \mathrm{eV}$ [3].

In conjugated polymers, the Peierls distortion is associated with an alternation of bond length along the carbon backbone that lowers the electron energy and can occur in two configurations. Figure 2.11 shows the two possible configurations for polyacetylene.

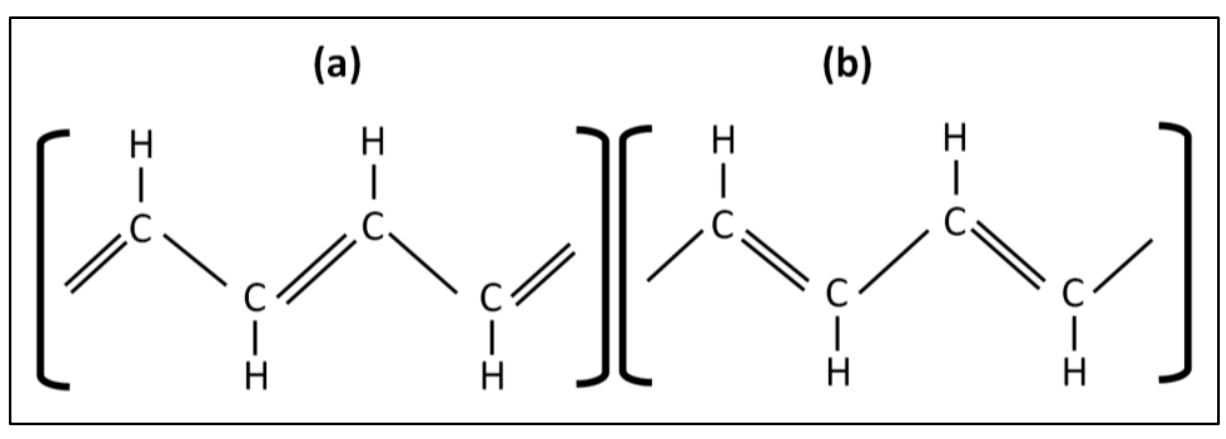

Figure 2.11 (a \&b) Two configurations for the Peierls distortion in polyacetylene 
The bond length depends on the type of bond, where a triple bond is the shortest and a single bond is the longest. When each of the two lattice distortion configurations lowers the energy of the lattice by the same amount then the polymer is degenerate and either configuration is equally likely to occur. Polyacetylene is a degenerate polymer and it has two ground states of the same energy.

If a lattice distortion configuration lowers the energy of the lattice more than the other configuration, then the polymer is nondegenerate and the former configuration is favoured over the latter. An example of a nondegenerate polymer would be poly $(3,4-$ ethylenedioxythiophene) (PEDOT) [7], the main material of interest in this work.

\subsubsection{Charge Carriers}

The doping of a conjugated polymer will introduce charge carriers that are not simply free electrons or holes. Instead, the added charge induces a charge-lattice coupling and that forms well-defined quasi-particles called solitons, polarons, or bipolarons. Due to a lattice polarization, these quasi-particles are self-localised and have electronic states called "gap states" located in the forbidden energy gap and where the doping charge is stored [4].

\subsubsection{Solitons}

In this section solitons are introduced using the definition of degenerate polymers, as explained in section 2.1.2.3, and polyacetylene is used as an example of this type of polymers. Polyacetylene has two bond alternation structures that are degenerate and hence are equally likely to occur. If one end of a polyacetylene chain has the first bond 
alternation and then switches to the second bond alternation as shown in Figure 2.12, then the location where the switch occurs will have a kink in bond alternation and a carbon atom that cannot form a $\pi$ bond with any of its neighbouring carbon atoms. Therefore there will be an unpaired $\pi$ electron with an unpaired $1 / 2$ spin in a nonbonding state that does not make part of neither the valence band nor the conduction band.

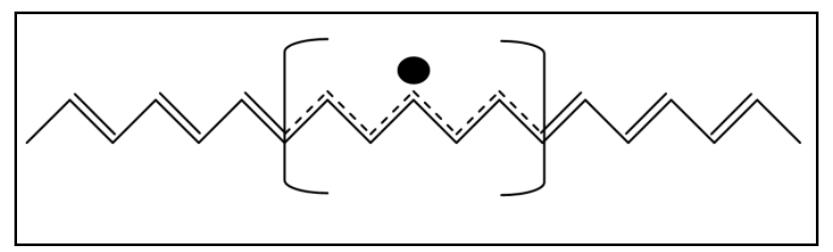

Figure 2.12 A kink in the bond alternation of a polyacetylene chain which creates a soliton and an unpaired electron pictured as a black dot

The kink in bond alternation is called a soliton and it is considered a self-localised state. Since the two bond alternations on either side of a soliton are degenerate in energy, the soliton can freely move along the polymer chain without creating a change in overall energy and is considered a mobile but neutral species. The spin and bond alternations on either side of a soliton are opposite to that of an anti-soliton. When a soliton is created, an energy state occupied by an unpaired $\pi$ electron appears at the center of the bang gap. This soliton has spin $1 / 2$ and zero charge since no charge was added to create the soliton. This gap state is made up of half contributions from the HOMO and LUMO bands. When a degenerate polymer is doped, it is more energy favourable for a $\pi$ electron to be removed from or added to an already existing soliton state than the HOMO or LUMO bands, respectively. In the case of $n$-doping/ $p$-doping, a $\pi$ electron is added to/removed from the neutral soliton. This means that the soliton state is full/empty, has charge of $-e / e$ and spin 0 . Therefore upon doping, charged solitons can become mobile charge carriers 
but with spin 0 , which explains the fact that spinless charged particles can be the cause for electrical conduction in conjugated polymers as previously mentioned. The spin and charge relation for the soliton are summarized in Figure 2.13 [1].

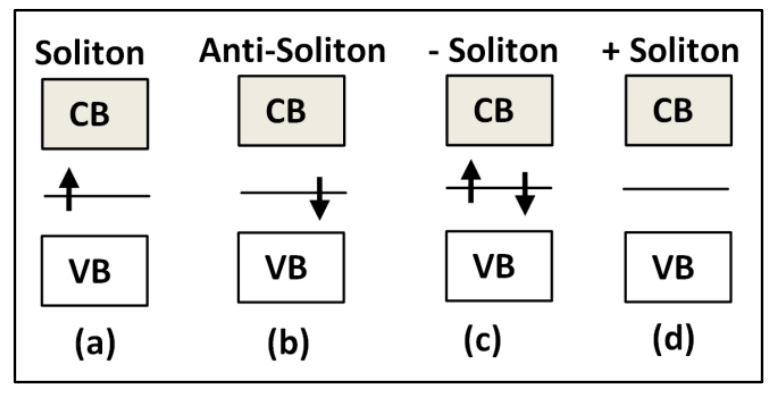

Figure 2.13 Spin and Charge relations for (a) a neutral soliton $[q=0, s=1 / 2]$, (b) anti-soliton $[q=0, s=$ $1 / 2]$, (c) negative soliton $[q=-e, \mathrm{~s}=0]$, and (d) positive soliton $[q=+e, s=0]$.

A soliton/anti-soliton pair would be made of two kinks where the bond alternation between the two kinks is different from the bond alternation outside of the kinks. In a degenerate polymer, a soliton/anti-soliton pair can exist separate from each other. However, in a nondegenerate polymer, the bond alternation between the two kinks has a higher energy than the outside bond alternation. Therefore as the separation between the soliton/anti-soliton pair increases, the total energy increases, and consequently, the soliton and anti-soliton cannot be separated from each other. As the soliton and antisoliton states come closer together they overlap and form antibonding and bonding states that make part of the LUMO and HOMO bands, hence the soliton/anti-soliton pair vanishes. Consequently, solitons cannot exist in nondegenerate polymers [3]. 


\subsubsection{Polarons}

When a degenerate polymer is $p$-doped ( $n$-doped) and there are no soliton states available, a soliton/anti-soliton pair is created and a $\pi$ electron is removed from (added to) either the soliton or anti-soliton. This results in a neutral soliton and positive (negative) soliton respectively. Although the neutral soliton and positive (negative) soliton are allowed to separate, they will come together and overlap into stable antibonding and bonding states that make up a positive (negative) polaron. This occurs because the polaron is a lower energy state than a separate neutral soliton and positive (negative) soliton. The creation of a polaron is depicted in Figure 2.14.

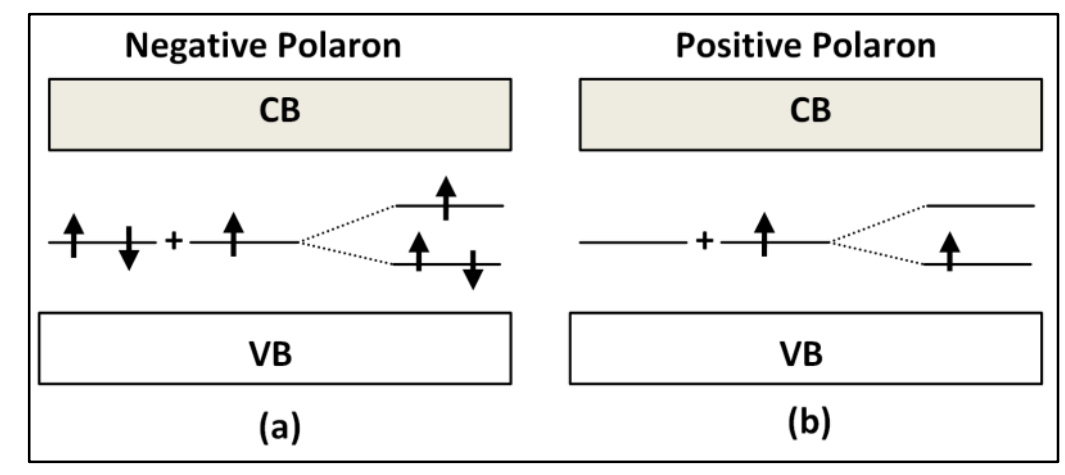

Figure 2.14 (a) Formation of a negative polaron $[q=-e, s=1 / 2]$ due to the overlap of a soliton and negative soliton. (b) Formation of a positive polaron $[q=+e, s=1 / 2]$ due to the overlap of a soliton and positive soliton.

These antibonding and bonding states do not make part of the LUMO and HOMO bands and are symmetric about the mid-band gap with the same energy separation from the LUMO and HOMO bands. In nondegenerate polymers, polarons can be created upon doping because a polaron localises the higher energy bond alternation to minimize the distortion of the lower energy bond alternation, unlike the case of a separated soliton/antisoliton pair [1]. 
In terms of conventional semiconductors, a polaron refers to a lattice polarization induced by an added charge. In the case of metals, there are many free electrons that can screen the polarization effect of added charge (i.e. an electron), but in semiconductors the lack of free charge cannot fully screen out the polarization of the lattice in the vicinity of the added charge. Moreover, the electron and the subsequent lattice polarization move together and this distortion will lower the electron energy with respect to the undisturbed case (i.e. no lattice distortion). This creates a potential well that will spatially localise the electron as long as the well is deep enough and the tails of the electron wavefunction do not significantly spread outside of the well. If the potential well has a width equal to the lattice constant, then the polaron is called a small polaron and is strongly localised. A large polaron has a wavefunction that spread over a few lattice units, hence it is not strongly localised and refers to the intermediate case between a small polaron and no polaron. In the case of small polarons, the charge transport of the localised electrons is explained by the hopping of charge into other localised states and not by the conventional energy band model in which the mean free path is much larger than the lattice spacing [1].

\subsubsection{Bipolarons}

In the case of low level doping, the added or removed charges will create polaron states, however, high level doping will allow for polaron states to combine and form bipolaron states. Similarly to a polaron, a bipolaron refers to a coupling of charge to a lattice distortion, but instead of a single charge, there is a pair of like charges. The bipolaron is a stable quasi-particle because the energy required to overcome the Coulomb 
repulsion between the pair of like charges is less than the energy gained by the lattice distortion around the pair of charges, which is analogous to the Cooper pair used to explain superconductivity. As two polarons come together, their energy states overlap and form the bipolaron states as shown in Figure 2.15 (a), which shows that the gap between the bipolaron states is smaller than the gap between polaron states. As more polarons combine to form new bipolarons, the two bipolaron states become bipolaron bands as shown in Figure 2.15 (b) [8].
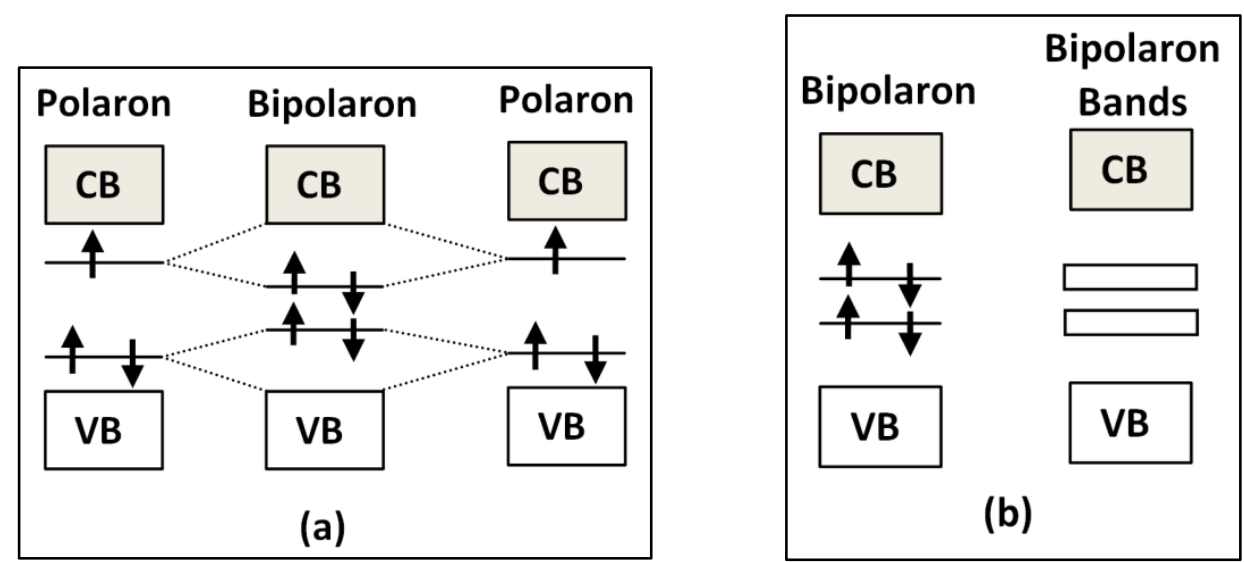

Figure 2.15 (a) Combination of two polarons to make a bipolaron. (b) Overlap of bipolaron states into the formation of bipolaron energy bands

Unlike solitons and polarons, a neutral bipolaron is not stable and hence a positive or negative bipolaron are the only stable form of this quasi-particle. A bipolaron has zero spin and once again allows for the conduction of spinless charged particles as previously seen in the case of solitons. This is the main conduction mechanism in PEDOT:PSS. The spin charge relations for bipolarons are summarized in Figure 2.16 [3]. 


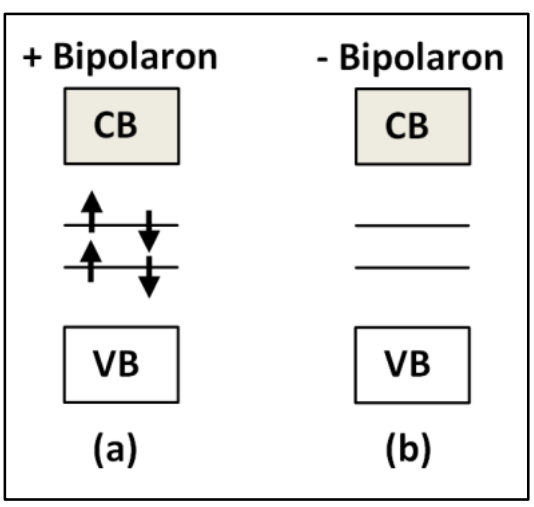

Figure 2.16 Illustration of a positive $[q=+e, s=0]$ and negative $[q=-e, s=0]$ bipolaron

\subsection{Electrochemical Systems}

\subsubsection{Introduction}

In a simplified model of mass transport of an electrolytic solution, the kinematics of electroactive species in an electrochemical system relies on the Nernst-Plank equation (equation 2.6) to describe how these species move when they experience electrical stimuli, concentration gradients and fluid flow [9]. This equation provides an expression for the flow rate per unit area $\left(N_{i}\right)$, or flux, $\left[\mathrm{mol} /\left(\mathrm{m}^{2} \bullet \mathrm{s}\right)\right]$ of each species based on transport mechanism of migration, diffusion and convection. In all the following equations, the " $i$ " subscript denotes each ion present in a multi-ion system.

$$
N_{i}=-D_{i} \nabla c_{i}-\mu_{i} c_{i} \nabla \varphi+c_{i} v
$$

In the equation above, $D_{i}$ is the diffusion coefficient $\left[\mathrm{m}^{2} / \mathrm{s}\right], c_{i}$ is the ion concentration $\left[\mathrm{mol} / \mathrm{m}^{3}\right], \mu_{\mathrm{i}}$ is the ion mobility $\left[\mathrm{m}^{2} /(\mathrm{V} \bullet \mathrm{s})\right], \varphi_{\mathrm{i}}$ is the electric potential [V] and $v$ is the fluid velocity $[\mathrm{m} / \mathrm{s}]$. The flux of species given by equation 2.6 can be used to determine the total current density $\left(J_{T}\right)$, in units of $\left[\mathrm{A} / \mathrm{m}^{2}\right]$, flowing through the 
electrolytic solution as shown in equation 2.7, where $F$ is the Faraday's constant [C/mol] and $z_{i}$ is the charge number [unitless].

$$
J_{T}=F \sum_{i} z_{i} N_{i}=-F \sum_{i} z_{i} D_{i} \nabla c_{i}-F \nabla \varphi \sum_{i} z_{i} \mu_{i} c_{i}+F v \sum_{i} z_{i} c_{i}
$$

In addition, the electroneutrality condition and the material balance equation also affect the manner in which these species behave. A material balance for a species regulates the accumulation, or time rate of change of concentration, of species in a given volume based on every flux coming into or out of the volume and the possible consumption and/or generation $\left(R_{i}\right)$ of species by means of homogenous chemical reactions [9]. This material balance also receives the name of continuity equation for the concentration of a species and is given by equation 2.8 .

$$
\frac{\partial c_{i}}{\partial t}=-\nabla \cdot N_{i}+R_{i}
$$

The electroneutrality condition on the species concentrations ensures that no excess charge density appears in the electrolyte without an externally applied electric field. The electroneutrality condition is given in equation 2.9 .

$$
\sum_{i} z_{i} c_{i}=0
$$

Using the equations 2.8 and 2.9 and assuming that all homogenous reactions in the solution are electrically neutral (i.e. for every cation consumed at one electrode another cation is produced at the other electrode, $R_{i}=0$ ), then one can conclude that equation 2.9 implies the conservation of charge, as shown by equation 2.10 [9]. 


$$
\begin{gathered}
F \sum_{i} z_{i} \frac{\partial c_{i}}{\partial t}=-F \sum_{i} z_{i} \nabla \cdot N_{i}+F \sum_{i} z_{i} R_{i} \\
F \frac{\partial}{\partial t} \sum_{i} z_{i} c_{i}=-\nabla \cdot\left(F \sum_{i} z_{i} N_{i}\right)+F \sum_{i} z_{i} R_{i} \\
\nabla \cdot\left(F \sum_{i} z_{i} N_{i}\right)=\nabla \cdot J=0 \\
\nabla \cdot J=0
\end{gathered}
$$

Although the electroneutrality condition is not a fundamental law of nature, its validity can be more clearly demonstrated by using the Poisson's equation given in equation 2.11 , where $\varepsilon$ is the permittivity $[\mathrm{F} / \mathrm{m}]$ of the electrolyte. The Poisson proportionality constant $\frac{F}{\varepsilon}$ is quite large $\left(1.09 \times 10^{16} \mathrm{~V} \cdot \mathrm{m} / \mathrm{mol}\right.$ in free space $)$ and hence it suggests that, in the absence of the electroneutrality condition, even a slight excess charge density would create a large electric field that would redistribute the charge and dissipate the excess charge density [9].

$$
\nabla^{2} \varphi(x)=-\frac{F}{\varepsilon} \sum_{i} Z_{i} c_{i}
$$

However, this electroneutrality condition does not apply near the electrodes and one must consider the ion concentration distribution away from the electrode and how it affects the voltage distribution. This region near the electrode is called the electric double layer (EDL) and will be explained in section 2.2.4 [9]. Furthermore, the electroneutrality condition ensures that the convection term in equation 2.7 does not contribute 
significantly to the overall current density and therefore it is ignored in any further analysis.

\subsubsection{Migration}

Ions in an electrolyte feel a driving force due to an electric field, similar to how an electric field causes the motion of mobile electrons in a metal. The total current density, given in equation 2.7, has a contribution due to migration of ions that is given by equation 2.12 , where $E$ is the electric field $[\mathrm{V} / \mathrm{m}]$ and $\sigma$ is the conductivity $[\mathrm{S} / \mathrm{m}]$ of the electrolyte [10].

$$
J^{\text {migration }}=-\left[F \sum_{i} z_{i} \mu_{i} c_{i}\right] \nabla \varphi=\sigma \mathrm{E}
$$

Since the electric field is equal to the negative gradient of the electric potential, one can see that equation 2.12 implies that Ohm's law applies to the electrolyte. Using Nernst-Einstein relation, given in equation 2.13 , the electrolyte conductivity $(\sigma)$ can be expressed in terms of the diffusion coefficient, concentration and charge number of each ion as shown in equation 2.14 [10]. In equation $1.13, q$ represents the ion charge, which equals the ion charge number $(z)$ times the elementary charge constant $(e), k_{B}$ is Boltzmann constant and $T$ is the temperature.

$$
\begin{gathered}
D_{i}=\frac{\mu_{i} k_{B} T}{q}=\frac{\mu_{i} k_{B} T}{z_{i} e} \\
\sigma=F \sum_{i} z_{i} \mu_{i} c_{i}=\frac{e F}{k_{B} T} \sum_{i} z_{i}{ }^{2} D_{i} c_{i}
\end{gathered}
$$


In the case of a binary electrolyte, i.e. an electrolyte with a cation and an anion as its only two dissociated ions, the conductivity expression simplifies to only two contributing terms. Furthermore, if one considers the anion to be essentially immobile, then the contribution of current carried by the cation is approximately the total current density. In this case, the conductivity further simplifies to equation 2.15 [10].

$$
\sigma=\frac{e F z_{+}^{2} D_{+} c_{+}}{k_{B} T}
$$

\subsubsection{Diffusion}

The presence of concentration gradient of a species in a solution induces the diffusion of this species which opposes any further increase of the concentration gradient. An example of this occurs at the electrode and electrolyte interface, where a species can be produced or consumed due to a chemical reaction and hence the species diffuse away or towards the electrode, respectively. This occurs in order to balance the difference between the concentration near the electrode and in the bulk solution. The process of mass transport due to diffusion of a species is described by two laws postulated by Adolf Fick. The first law relates the flux of species to the gradient of the species concentration as shown in equation 2.16. Meanwhile, the second law relates the Laplacian of the species concentration to the time derivative of the species concentration as shown in equation 2.17 [9].

$$
-N_{i}(x, t)=D_{i} \nabla c_{i}(x, t)
$$




$$
\frac{\partial c_{i}(x, t)}{\partial t}=D_{i} \nabla^{2} c_{i}(x, t)
$$

In both cases the constant of proportionality is the diffusion coefficient, which has been assumed to be constant. Although, diffusion applies to both neutral and charged particles, in the case of charged particles, Fick's laws ignore any particle-particle interaction due to Coulomb forces.

\subsubsection{Electric Double Layer}

The interface between an electrode and an electrolytic solution can give rise to an electric double layer (EDL) due to an applied potential or the tendency of a particular ion to be close to the electrode surface [9]. Helmholtz modeled the EDL using a parallel-plate capacitor made up of a charge separation between the surface charges in the electrode and the ion layer in the solution. This charge separation, called the Helmholtz layer, is in the order of the ion radius and the permittivity of the solution is assumed to be constant within this distance. However, this model ignores any effect due to the electrolyte concentration and the thermal motion of the ions that tends to loosen the ion layer [10].

When the thermal motion of ions is taken into account, a diffuse double layer is formed and it depends on the electrolyte concentration. Therefore the double layer acquires a variable thickness unlike the Helmholtz model. Gouy and Chapman introduced this diffuse double layer model in which they used the Boltzmann law to describe the distribution of ions as a function of distance away from the electrode [10]. The diffuse layer thickness in this model, usually referred to as the Debye length, represents the distance from the electrode surface over which the ion concentration drops to $1 / \mathrm{e}$ of its 
value at the electrode surface. For a symmetric binary electrolyte (i.e. an electrolyte with one type of cation and one type of anion that dissociate in equal amounts) the magnitude of the charge numbers for the cation and the anion will be equal and the bulk concentrations of both ions can be assumed to be the same. In this case the Debye length ( $\lambda$ ) can be derived using the Boltzmann law, the Poisson equation and the Debye-Hückel approximation in order to obtain the results shown in the equation below.

$$
\lambda=\sqrt{\frac{\varepsilon_{r} \varepsilon_{o} k_{B} T}{2 e z^{2} F c_{o}}}
$$

In equation $2.18, \varepsilon_{\mathrm{r}}$ and $\varepsilon_{0}$ represent the dielectric constant and permittivity of free space, respectively, and $c_{o}$ is the bulk electrolyte concentration $\left[\mathrm{mol} / \mathrm{m}^{3}\right]$. As mentioned before, the thermal motion of ions will tend to stretch the diffuse layer thickness which is seen in the square root dependency of the temperature $(T)$ on the Debye length in equation 2.18.

\subsubsection{Solvation Effects}

Polar solvents are characterized by a high dielectric constant, which means that dipoles can be induced in the polar solvent molecules due to an applied electric field or a charged particle such as an ion. When a solute is dissolved into a polar solvent there can be interactions between the dissociated ions from the solute and the polar molecules from the solvent. These interactions are usually electrostatic ion-dipole forces in which the solvent molecules form dipoles and surround the dissolved ion, which results in the formation of solvation sheaths around the ion [11]. A typical sign that solvation is taking place when dissolving a solute into a solvent is the production of heat that indicates an 
exothermic reaction. The solvation sheaths are divided into a primary sheath and a secondary sheath. The primary sheath is characterized by its close proximity to the ion and strong ion-dipole interaction, which means that, when the ion moves, the ion carries the entire sheath with it [11]. Therefore, the ion has a large effective radius and its mobility is reduced. In the secondary sheath the ion-dipole interaction weakens with increasing distance from the ion. Overall, when an ion is introduced into a solvent it disturbs the arrangement of solvent molecules in the vicinity of the ion, which is analogous to a polaron or an electron that carries a lattice deformation as it moves through the lattice.

In the case of lithium perchlorate $\left(\mathrm{LiClO}_{4}\right)$ dissolved in propylene carbonate, the main electrolyte used in this work, several authors have investigated the $\mathrm{Li}^{+}$ion solvation by means of spectroscopic methods, such as Infrared (IR) and Raman spectroscopy. In this case, the interaction between the $\mathrm{Li}^{+}$ion and the solvent molecules is monitored by changes in vibration frequencies and/or band intensities. Xuan et al. (2001) showed that the solvation of lithium ion would occur due to an interaction with the carbonyl oxygen atom in propylene carbonate. This interaction between the lithium ion and the solvent would manifest itself in the IR spectra as a new band whose intensity depended on the $\mathrm{LiClO}_{4}$ concentration. Additionally, Raman and IR spectra changes also suggest a possible weak interaction between the $\mathrm{Li}$ ion and the ring oxygen of the solvent molecule that is also concentration dependent. Xuan et al. (2001) also suggest that as the concentration of $\mathrm{LiClO}_{4}$ increases, the $\mathrm{Li}$ ion becomes bound to the $\mathrm{ClO}_{4}$ ion and create a solvent separated ion pair, which leads to higher viscosity and lower ion mobility [12]. 
These solvation effects affect the measured ion conductivity and influence electrolytic device operation.

\subsection{Nuclear Magnetic Resonance (NMR)}

\subsubsection{Introduction}

Quantum mechanics describes an atom based on discrete quantum numbers, which include the orbital angular momentum. The angular momentum of a charge particle, such as an electron, will produce a magnetic moment, similar to the magnetic moment produce by an electrical current flowing in a circular wire path. Using a classical treatment, one can define the magnetic dipole moment of the electron based on the picture of a charge particle revolving around the nucleus in a circular orbit [13]. Although this is not the quantum mechanical treatment, one can obtain a valid quantum mechanical relation between angular momentum and magnetic dipole moment $(\widehat{\boldsymbol{\mu}})$, shown in equation 2.19 ,

where $\mu_{\mathrm{B}}$ is the Bohr magneton, $\widehat{\boldsymbol{L}}$ is the orbital angular momentum vector operator, and $g$ is the orbital g-factor and $\mathrm{h}$ is Planck's constant [13]. The Bohr magneton is given in equation 2.20 where $m_{e}$ is the mass of an electron and $e$ is the elementary charge constant.

$$
\begin{gathered}
\hat{\mu}=-g \mu_{B} \frac{\hat{L}}{\hbar} \\
\mu_{B}=\frac{e \hbar}{2 m_{e}}
\end{gathered}
$$

There is another intrinsic quantum mechanical property of the electron called spin, which endows the electron with an additional magnetic dipole moment. This magnetic 
dipole moment associated with the spin of a particle $\left(\widehat{\mu_{S}}\right)$ is given by equation 2.19 , but $\widehat{L}$ is replaced by the spin vector operator $\hat{S}$ and $g$ is replaced by the spin g-factor $\left(g_{S}\right)$ [13].

The property of spin is not exclusive to an electron; instead it also applies to the nuclei of an atom. Both protons and neutrons have a nuclear spin which gives rise to a nuclear magnetic dipole moment. This nuclear magnetic dipole moment $\left(\widehat{\boldsymbol{\mu}_{\boldsymbol{I}}}\right)$ is also given by equation 2.19 , but $\hat{L}$ is replaced by the nuclear spin vector operator $\widehat{\boldsymbol{I}}, g$ is replaced by the nuclear g-factor $\left(g_{N}\right)$ and $\mu_{\mathrm{B}}$ is substituted by the nuclear magneton $\left(\mu_{\mathrm{N}}\right)$ [13].

Although, a neutron does not have charge, its constituent particles called quarks do have charge and hence the spin of a neutron is made up of a combination of quark spins. For the purpose of NMR applications, the proton and neutron can be assumed to have a well-defined spin-1/2 [14].

\subsubsection{Magnetic Field Interaction}

If one places a hydrogen atom in a constant homogenous magnetic field, the single proton in the nucleus interacts with this magnetic field by means of its nuclear magnetic dipole moment. This interaction involves a magnetic potential energy that is added to or subtracted from the usual Coulomb potential energy. In the case of a magnetic field with only a $z$ component of magnitude $B_{o}$, this magnetic potential energy only involves the expectation value of the $z$ component of the nuclear spin vector operator, which is given by Planck's constant times the nuclear spin quantum number $(i)$. This magnetic potential energy is given by equation 2.21 where $\mu_{\mathrm{N}}$ is the nuclear magneton and $g_{N}$ is the nuclear g-factor [15].

$$
V_{M}=-\hat{\mu}_{I} \cdot \hat{B}=-g_{N} \mu_{N} \frac{\left\langle\hat{I}_{z}\right\rangle B_{o}}{\hbar}
$$




$$
V_{M}=-g_{N} \mu_{N} i B_{o}
$$

A proton has a nuclear spin quantum number of $\pm 1 / 2$, therefore the energy of the proton is lowered or raised by a constant $\delta E$ that depends only on the applied magnetic field strength. Therefore, the degeneracy of the energy level caused by spin is broken and the energy level splits into two levels with an energy gap equal to $\Delta E$. Using the PlanckEinstein equation for the energy of a photon, one can determine the frequency $(\omega)$ of a photon required for a proton to make a transition from a state of spin $-1 / 2$ to spin $1 / 2$ or vice versa, which is given by equation 2.22 [15].

$$
\begin{gathered}
\Delta E=g_{N} \mu_{N} B_{o}=-\gamma_{N} \hbar B_{o}=\hbar \omega \\
\omega=-\gamma_{N} B_{o}
\end{gathered}
$$

The gyromagnetic ratio or magnetogyric ratio $\left(\gamma_{N}\right)$ is sometimes preferred instead of the nuclear g-factor and nuclear magneton [15]. The frequency given in equation 2.22 is sometimes called the Larmor precession frequency which, in classical physics, represents the frequency of the precessional motion of a magnetic dipole moment about the fixed axis of an applied magnetic field [15].

\subsubsection{Net Magnetization Dynamics}

Spin is usually visualized as a vector in space whose direction is called the spin polarization axis and can point in any direction in space. If a proton has a spin polarization along the $+z$ axis and one were to measure of its spin along the $z$ axis then the result would always be $1 / 2 \hbar$ or spin up (the negative $z$ axis polarization would be $-1 / 2 \hbar$ or spin down) and the spin vector would be depicted as an vector arrow pointing in the $+z$ 
axis. However, this vector picture does not imply that a measurement of the spin along the $x$ or $y$ axis of the same proton would be zero, instead the result would be either $1 / 2 \hbar$ or $-1 / 2 \hbar$ with equal probability for each result. Therefore the vector picture of spin is incomplete in the sense that it does not fully describe the nature of spin and caution must be taken when using this pictorial device [14].

In the case of a water sample without an external magnetic field, the net spin polarizations are isotropic or uniformly distributed and no particular direction is favoured as shown in Figure 2.17.

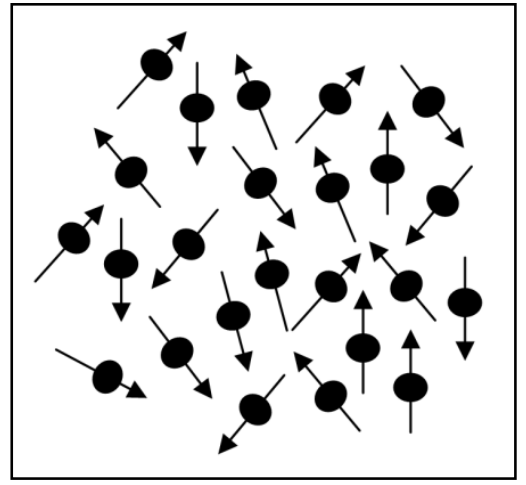

Figure 2.17 An isotropic or uniformly distributed ensemble of spin polarizations

When there is a static magnetic field the spin vectors begin to precess about the direction of the magnetic field (usually defined as the $z$ axis). However, an individual spin is surrounded by electrons and other nuclei that have their own magnetic moments and cause fluctuations in the magnetic field that the spin in question experiences. Due to these slight variations in the surrounding magnetic fields of each proton (also referred as chemical shift) the axis of precession varies over time and cause the isotropy of the net spin polarization to be disturbed. The net spin polarization becomes anisotropic because, as the axis of precession of each spin vector varies, each spin vector eventually favours 
the axis that lowers its energy which would be orientated parallel to the applied magnetic field. The overall effect is that in thermal equilibrium there is on average a larger amount of spin polarizations parallel to the magnetic field and hence there is a net magnetic moment along this direction called the longitudinal magnetic moment (Figure 2.18). The longitudinal magnetic moment is quite small and almost undetectable for any practical NMR purposes [14].

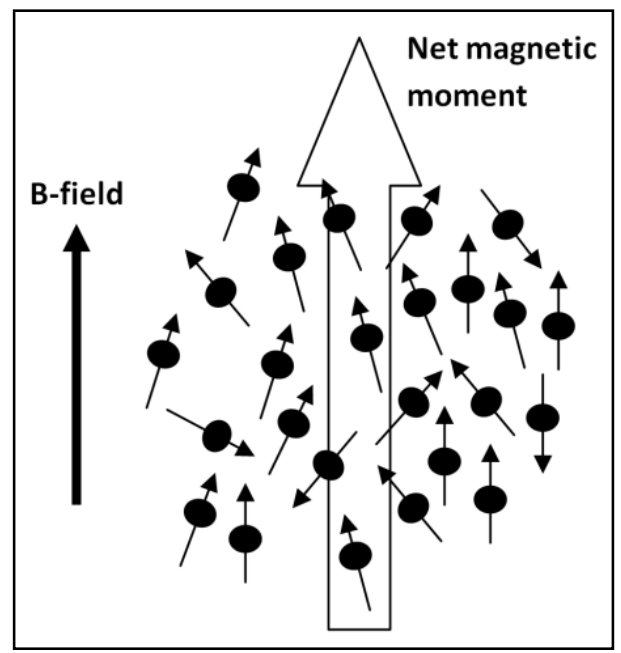

Figure 2.18 An anisotropic ensemble of spin polarization constituting a net longitudinal magnetic moment

In NMR experiments another net magnetic moment, called the transverse magnetization, is developed in the sample by applying an electromagnetic radio frequency (RF) pulse of appropriate amplitude, frequency, phase and duration to the sample. This RF pulse is applied using a coil that is perpendicular to the static magnetic field (z-axis), thus the coil axis is assumed to be the $x$-axis. The effect of the pulse, as shown in Figure 2.19, is that it rotates the net magnetic moment about the axis of the RF coil (x-axis) and the angle of rotation depends on the amplitude and duration of the RF pulse. 


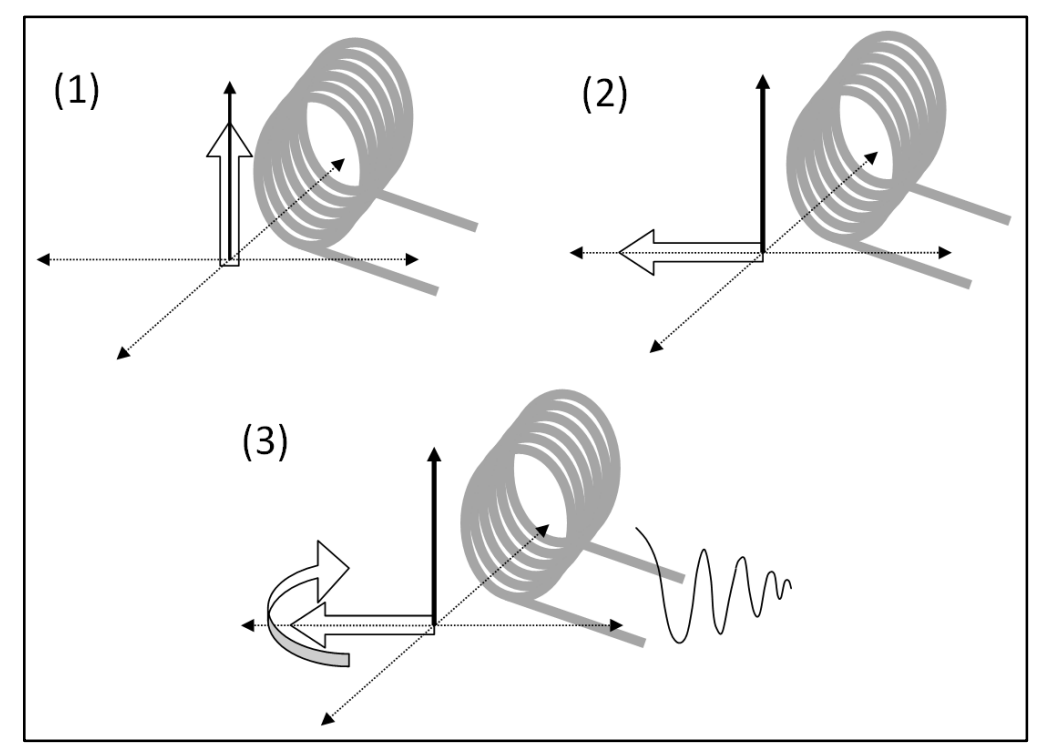

Figure 2.19 Rotation of the net magnetic moment about the RF coil axis by 90 degrees

In order to maximize this rotation of the net magnetic moment the frequency of the RF pulse must be equal to the resonance or Larmour frequency (equation 2.22), otherwise the RF pulse does not effectively rotate the net magnetic moment. In NMR jargon a $\pi / 2$ pulse rotates the net magnetic moment by $90^{\circ}$, which in this case corresponds to the $y$ axis. Once the RF $\pi / 2$ pulse is removed the transverse magnetization begins to precess the static magnetic field in the $x-y$ plane with precession frequency given by equation 2.22 . However, the transverse magnetization slowly decays to zero as the precession coherence of the precessing spins is disturbed by microscopic fluctuations (chemical shift) in the surrounding magnetic field of each spin, as previously explained. This decay process is called Transverse Relaxation Time Constant or $T_{2}$ relaxation. As the transverse magnetization decays, the precession of the transverse magnetization generates a decaying oscillating magnetic field, which then generates a decaying oscillating electric field that can be detected using a sensitive (RF) detector in the form of a decaying 
oscillating current. This electric current is called the Free-Induction Decay (FID) signal and is the basis for more complicated NMR experiments. Once the FID is acquired, a Fourier transform is applied to determine the frequency content of the signal and perform further analysis [14].

\subsubsection{Pulsed Gradient Spin Echo NMR}

In a NMR sample the molecular surroundings (chemical shift) of each spin plays an important role as previously mentioned. In addition to this, the particles or molecules in the sample are in continuous motion, as a result, the magnetic surroundings of each spin is constantly varying and the effect this has on the NMR signal can allow one to measure how a particle is moving. Hence the measurement of self-diffusion is possible by using a special NMR pulse sequence called Pulsed Gradient Spin Echo (PGSE) [14].

The PGSE technique uses two RF pulses (a $\pi / 2$ and $\pi$ pulses), a static magnetic field and a gradient magnetic field in order to create an echo of the FID signal. The gradient magnetic field $\left(G_{z}\right)$ and static magnetic field $\left(H_{z}\right)$ point in the $z$ direction. At thermal equilibrium, a water sample placed under $H_{z}$ has a net magnetization vector $M_{o}$ pointing towards the $z$ axis [14]. The first $\pi / 2$ pulse rotates $M_{o}$ to the $-y$ axis. If one considers particles that do not diffuse (i.e. a viscous substance), once the $\pi / 2$ pulse is turned off, $M_{o}$ decays only due to $T_{2}$ relaxation. After a time delay of $\tau$, a $\pi$ pulse is applied that flips each spin vector about the $x$ axis by $180^{\circ}$. Because each spin precesses at the same frequency and direction as it did prior to the $\pi$ pulse, all the spins refocus after the pulse is applied. This in turn causes the precession coherence to build up and the net magnetization vector to grow to its original value of $M_{o}$. This net magnetization vector 
will decay back down to zero [16]. This is called the echo signal and its maximum intensity is given by equation 2.23 as a function of the $T_{2}$ relaxation and time delay $(\tau)$ between the $\pi / 2$ and $\pi$ pulses.

$$
M_{y}(\tau)=M_{o} e^{-\left[\left(\frac{\tau}{T_{2}}\right)\right]}
$$

If one considers the diffusion of particles, an additional exponential decay must be included to equation 2.23. This diffusion term takes into account that as particles diffuse they experience a different magnetic field since $G_{z}$ changes with position and in turn this increases the rate at which the precession coherence is lost among all the spins. The final equation for the maximum echo signal is given by equation 2.24 , which includes the diffusion coefficient $(D)[16]$.

$$
M_{y}(\tau)=M_{o} e^{-\left[\left(\frac{\tau}{T_{2}}\right)+\left(\frac{\gamma^{2} G_{z}^{2} D \tau^{3}}{12}\right)\right]}
$$

Steady magnetic field gradients are sometimes replaced by pulsed magnetic field gradients that have a field strength $\widehat{G}_{z}$, time duration $\delta$ and a time separation $\Delta$ as shown in Figure 2.20 below. In this case, the maximum echo signal magnitude is given by the Stejskal-Tanner equation (equation 2.25) [17]. 


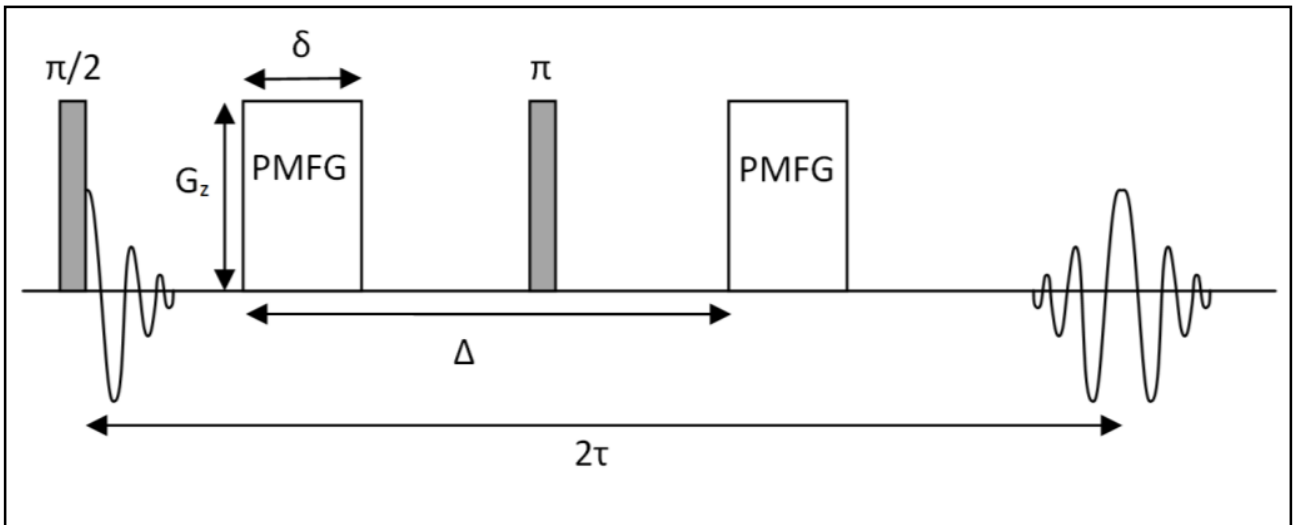

Figure 2.20 Time diagram of a PGSE-NMR experiment using pulsed magnetic field gradients

$$
M_{y}(\tau)=M_{o} e^{-\left[\left(\frac{\tau}{T_{2}}\right)+\left(\frac{\gamma^{2} \widehat{G}_{z}^{2} \delta^{2}(\Delta-\delta / 3) D}{12}\right)\right]}
$$

Therefore it is easy to see that by varying one of the parameters in the exponent, namely $\widehat{G}_{z}, \delta$ or $\Delta$, the diffusion coefficient can be extracted by fitting the experimental data for the maximum amplitude of the echo signal to equation 2.25. 


\section{Chapter 3}

\section{Literature Review}

\subsection{Material Properties of PEDOT:PSS}

There are several conjugated polymer families that have been developed over the past 25 years which include polyanilines, polypyrroles, polythiophenes, polyphenylenes, and poly(p-phenylene vinylene)s [18]. In the case of polyaniline one can obtain low cost processable conductive forms, but toxic products may result upon polymer degradation due to the presence of benzidine moieties. The other polymer families are safer but they are insoluble which limits their use in film forming applications [18]. In the 1980s, Bayer AG research laboratories developed a water-soluble polyelectrolyte called PEDOT:PSS which was composed of a polythiophene derivative called poly $(3,4-$ ethylenedioxythiophene) (PEDOT) and poly(4-styrenesulfonate) (PSS) and is shown in Figure 3.1 . 


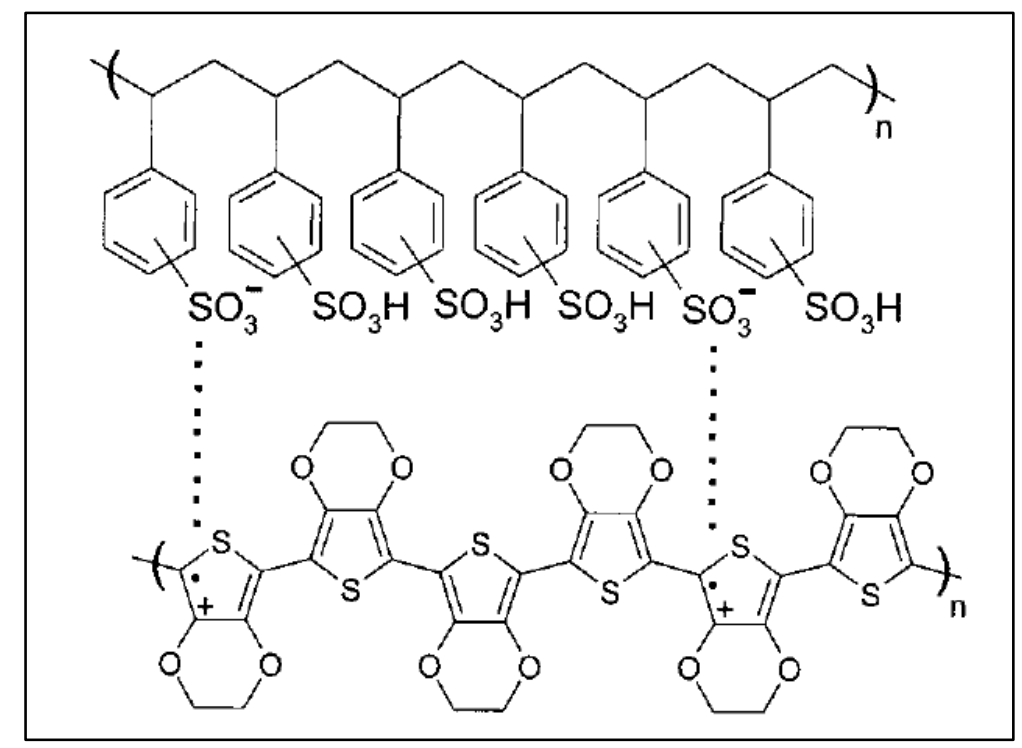

Figure 3.1 Chemical structure of PEDOT:PSS made up of a PSS backbone (top) and a PEDOT chain (bottom) ionically bonded (with permission from [19])

PEDOT:PSS has found a diverse array of applications ranging from antistatic coatings, electroluminescent devices, hole injecting layer in Organic Light Emitting Diodes (OLEDs) [20], electrochromic devices or pixel displays [21] [22], and organic transistors [23] among others. One reason for the broad range of applications of PEDOT:PSS is that it can be implemented by a variety of printing techniques including ink-jet, screen printing, spin coating, vacuum deposition and graphics art printing [24].

PEDOT is referred as a polycation because each ethylenedioxythiophene (EDOT) monomer can lose a $\pi$-electron and have a positive charge. Meanwhile PSS is a polyanion since each $\mathrm{SO}_{3} \mathrm{H}$ functional group can lose its hydrogen atom making the PSS have a negative charge as shown in Figure 3.1. The PSS polyanion can oxidize or $p$-dope the EDOT monomer and create an ionic bond between the two. Therefore, it is possible to grow EDOT oligomers, or PEDOT chains, along the PSS polymer backbone as these EDOT oligomers are oxidized and attached onto the PSS. In a PEDOT chain there is a 
hole for each ionic bond between the PSS backbone and the PEDOT chain [20]. Therefore, the PSS polymer is used as a template polymer on which the polymerization of EDOT monomers can take place to form conductive $p$-doped PEDOT chains [20]. The choice of PSS as the polycounter ion is due to its solubility in water and ability to form durable and transparent films [7].

According to mass spectroscopy, these individual PEDOT segments are short (6 to 18 repeating units) and therefore the structural model of PEDOT:PSS is seen as short PEDOT oligomer segments tightly attached to a long (higher molecular weight) PSS chain as shown in Figure 3.2(b). These PEDOT:PSS chains form entangled particles consisting of approximately 90 to $95 \%$ water which allows for a printable dispersion (Figure 3.2(d)) [20].

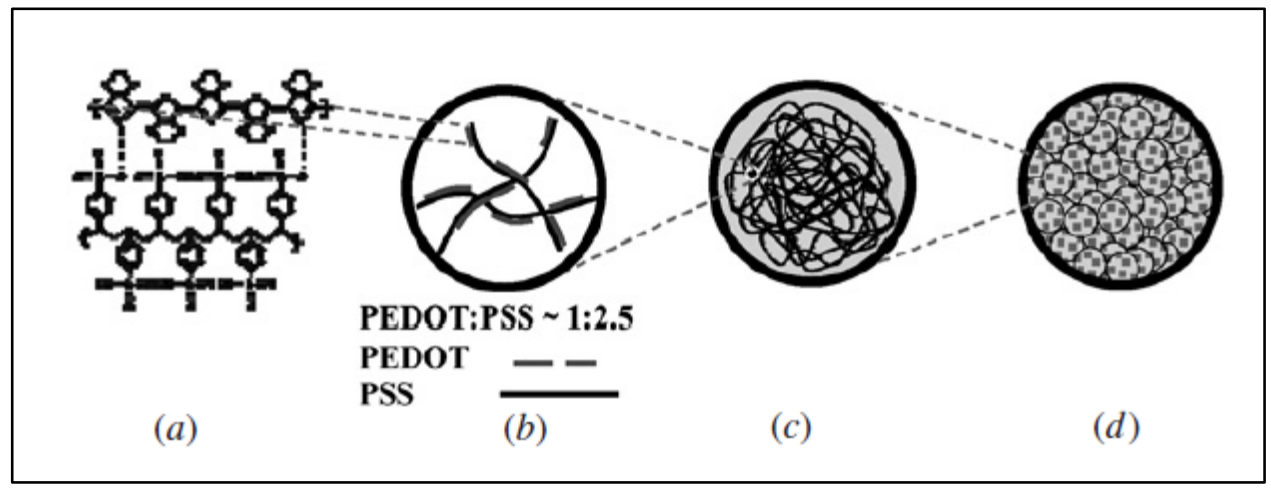

Figure 3.2 (a) Chemical structure of PEDOT:PSS. (b) Individual PEDOT chains attached to long PSS chains. (c) Entangled PEDOT:PSS chains. (d) Particles of entangled PEDOT:PSS chains (with permission from [19])

\subsection{Morphology}

PEDOT:PSS films are also known to have anisotropic morphology. For example, Timpanaro et al. (2004) performed studies on the morphology of spin-coated PEDOT:PSS films using STM and AFM and showed that these films have a lamellar 
structure made up of PEDOT clusters embedded in a PSS matrix. The AFM and STM study also revealed that the morphology of the surface and bulk of the film have very different PEDOT cluster size and composition [25]. In a similar study, Nardes et al. (2007) used phase-sensitive AFM on cryogenically cleaved samples to show that PEDOT:PSS films have a granular morphology and a lamellar structure (Figure 3.3), where PEDOT-rich particles have a diameter of 20-30 nm (in-plane) and a height of 4-6 nm (out-of-plane). These oval-like PEDOT-rich grains were separated by quasicontinuous PSS lamellas in the out-of-plane direction and had no sharp separations along the in-plane direction [26]. It is important to highlight that the PEDOT:PSS films used in this thesis are screen-printed, whereas the previously mentioned studies deal with spincoated films. Nevertheless, a degree of anisotropy is expected to exist in screen-printed films as well.

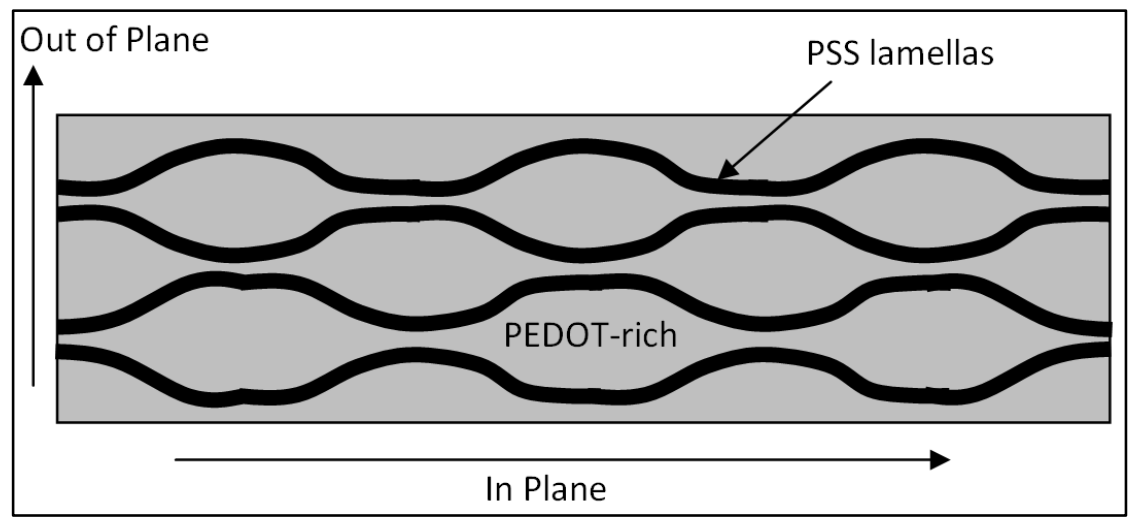

Figure 3.3 Lamellar structure of PEDOT:PSS illustrating the granular morphology made up of PEDOT-rich regions separated by PSS lamellas 


\subsection{Charge Transport Model}

Based on Scanning-Tunneling Microscopy studies done on PEDOT:PSS films, a PEDOT:PSS film can be pictured as mixture of conductive regions or domains that are PEDOT-rich and non-conductive domains that do not have PEDOT particles and are made up of only the PSS backbone [25]. The conduction of current consists on the transfer of charge carriers among PEDOT-rich domains. Hence, the ratio of PEDOT to PSS, or in other words the density of PEDOT-rich domains, will be a significant factor in determining the overall conductivity of the PEDOT:PSS film [27]. By the same token, the conductivity can also decrease by adding other foreign nonconductive particles to the PEDOT:PSS mixture [7]. Overall, the morphology of the polymer film will determine the distribution of these conductive and nonconductive domains and therefore it is a crucial parameter in determining the conductivity and charge transport of the film [25].

Besides the morphology of the film, the conductivity of PEDOT:PSS also depends on factors such as the type of polymer used as a backbone, level of doping, and the ion used to dope the polymer. Despite the wide range of applications in which PEDOT:PSS is being currently used, a clear and well-understood model for the conductivity of this conjugated polymer is still missing [28]. The inherit disorder of conjugated polymers does not allow one to apply the models used in crystalline inorganic semiconductors and metals [7]. In these models the electron experiences little scattering due to the translation symmetry of the lattice, has a well-defined momentum and is delocalised. As disorder is introduced into this type of system, the electron encounters more scattering, as a result, the conductivity and mean free path decreases. Eventually, as the mean free path length becomes comparable to the lattice constant, the electron no longer has a well-defined 
momentum and it is spatially localised. However, an electron can still move among these localised states by means of thermally activated hopping [3].

Thus, the conductivity of disorder systems, such as conjugated polymers, is more appropriately explained using a model used in amorphous inorganic semiconductors in which charge hopping is the determining factor on the conductivity [7]. In the literature, a model called Variable Range Hopping (VRH) is commonly used to describe the charge transport in PEDOT:PSS films [29] [28].

Based on the previously mention anisotropic morphology of PEDOT:PSS, a study by Nardes et al. (2007) investigated the in-plane and out-of-plane conductivities of spin coated PEDOT:PSS films and concluded that the conduction mechanism for the in-plane and out-of-plane directions was due to 3-dimensional VRH and nearest-neighbour Hopping (nn-H), respectively [28]. Overall, the conduction of charge in conjugated polymers is very different from band transport, commonly used for inorganic semiconductors. The transport of charge in conjugated polymers occurs in mid-band gap states, instead of the conduction or valence band, and the charge carriers are the previously mentioned quasi-particles and not electrons or holes [8].

\subsection{Doping}

Analogous to inorganic semiconductors, conjugated polymers can be $p$-doped or $n$ doped by losing or gaining a $\pi$ electron, respectively. This greatly improves their conductivities from their pristine conductivity [7].

The doping of conjugated polymers was thought to occur by adding or removing electrons from the conduction (LUMO) and valence (HOMO) bands, respectively, in 
analogy to the doping of inorganic semiconductors. However, in conjugated polymers it may be more energy favourable to localise the dopant charge through a lattice deformation. This dopant charge is stored in energy levels located in the bandgap, instead of the LUMO or HOMO bands, and this leads to the formation of quasi-particles called polarons, bipolarons and solitons. These "gap states" and quasi-particles are introduced and explained in detail in section 2.1.3. This mechanism for the doping of conjugated polymers was reinforced after the discovery that electrical conduction in conjugated polymers could occur due to spinless charged particles, such as solitons or bipolarons, and not spin $1 / 2$ particles such as electrons or holes [8]. These quasi-particles are delocalised over a few monomer units along the polymer chain. Due to the short conjugation length of the polymer with respect to the delocalization length of the quasiparticles [30], a single polymer chain can remain well conducting even when singly ionized. On the other hand, the transfer of charge carriers among conducting polymer chains will occur by means of charge hopping. Therefore, the interchain conductivity will dominate the intrachain conductivity [19].

Upon doping, the $\pi$ electron is removed or stored in novel states that include solitons, polarons, or bipolarons [7]. However, the $\pi$ electron is removed or added to the $\pi$ system of the conjugated polymer through oxidation or reduction of the polymer, respectively, and not by inserting an impurity atom to the lattice which is the case in inorganic semiconductors. The oxidation or reduction of the polymer occurs by means of a redox reaction, which refers to oxidation and reduction reactions. Doped conjugated polymers can increase their conductivities by several orders of magnitude. A conjugated polymer 
can be doped by chemical doping, electrochemical doping, photo-doping or charge injection. The technique used in this work was electrochemical doping.

\subsubsection{Electrochemical Doping}

A conjugated polymer can be electrochemically doped by using an electrolytic cell made from two electrodes connected together by a liquid or solid electrolyte as shown in Figure 3.4.

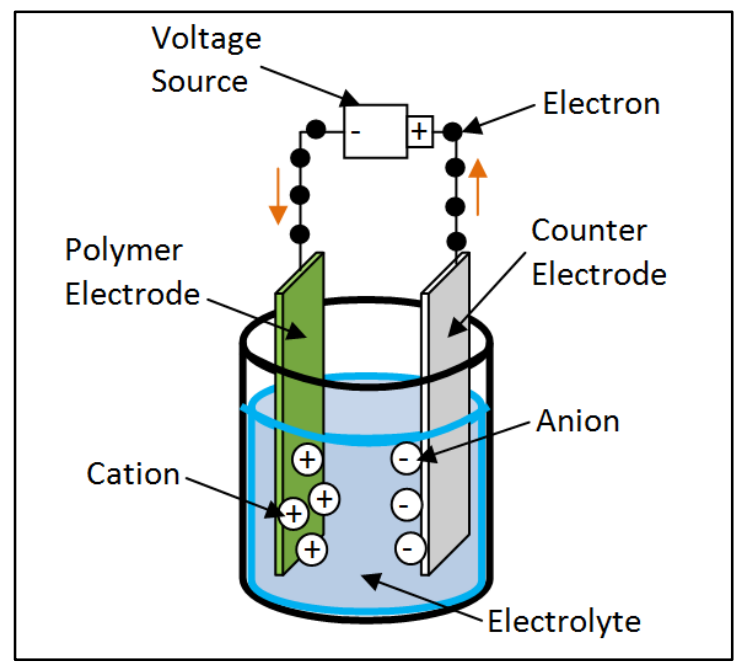

Figure 3.4 Illustration of an electrolytic cell and its components

One of the electrodes is made from a metal covered by a layer of the conjugated polymer and the other electrode can be made from any metal that would not be corroded by the electrolyte. The electrolyte refers to a liquid or gel that contains mobile ions that can oxidize or reduce the polymer. If a voltage difference is applied between the two electrodes, the cations and anions in the electrolyte are attracted towards the low voltage electrode (anode) and the high voltage electrode (cathode), respectively [3]. As the cations and anions separate, electrons can come in to the anode and exit from the cathode, 
thereby creating an electrochemical current. Hence, the electrons do not physically travel from the anode to the cathode by passing through the electrolyte.

In chemical and electrochemical doping, a counter-ion that functions as an oxidizing or reducing agent is intercalated into the polymer and causes oxidation or reduction, respectively [3]. In the case of PEDOT:PSS, when polystyrenesulfonic acid (PSSA) loses a hydrogen atom and becomes an oxidizing counter-ion ( $\left.\mathrm{PSS}^{-}\right)$, it oxidizes PEDOT by forcing it to lose a $\pi$ electron and become p-doped (equation 3.1).

$$
\text { PEDOT }^{0}+\mathrm{PSS}^{-} \rightarrow\left(\mathrm{PEDOT}^{+}: \mathrm{PSS}^{-}\right)^{0}+\mathrm{e}_{\pi}^{-}
$$

The PSS ${ }^{-}$counter-ion and the doped PEDOT $^{+}$can ionically bond and form a stable structure. This p-doping of PEDOT $^{+}$by the PSS $^{-}$counter-ion can be reversed by introducing a $\mathrm{Li}^{+}$cation, or other monovalent cation. The cation breaks the ionic bond between the PEDOT $^{+}$and PSS $^{-}$and neutralizes the PSS $^{-}$polyanion, while the PEDOT $^{+}$ polycation gains an electron and becomes dedoped. Hence, the $\mathrm{Li}^{+}$cation causes the reduction of the $p$-doped PEDOT segment as shown in equation 3.2. This redox reaction is the basis of the dedoping of PEDOT:PSS that is used in this work.

$$
\left(\mathrm{PEDOT}^{+}: \mathrm{PSS}^{-}\right)^{0}+\mathrm{Li}^{+}+\mathrm{e}_{\pi}^{-} \rightarrow \mathrm{PEDOT}^{0}+\left(\mathrm{Li}^{+}: \mathrm{PSS}^{-}\right)^{0}
$$

In addition, the electrolytic cell shown in Figure 3.4 has a capacitance associated with the amount of ionic charge stored at the electrodes. Therefore, the redox reaction given in equation 3.2 can also be explained as the charging of the double layer (section 2.2.4) between a PEDOT:PSS electrode and the electrolyte with an associated RC time constant. 


\subsection{Conductivity vs. Ion Concentration}

Since the conductivity of conjugated polymers depends on the doping level or redox state of the polymer, the modeling of a conjugated polymer requires knowledge of this dependency. Several studies have determined the conductivity of a conjugated polymer as a function of dopant concentration or an equivalent measure of doping level using X-ray fluorescence spectroscopy (XFS) [31], elemental analysis [32], and Electrochemical Quartz Crystal Microbalance (EQCM) [33] [34].

Berzina et al. (2009) used a three terminal device to show that the conductivity of a polyaniline (PANI) film decreased as a function of transferred ionic charge into the PANI film, which was done by integrating the gate current. They also used XFS to further show that the drain current across their PANI film decreases as Rubidium $(\mathrm{Rb})$ ions are intercalated into the PANI film which causes reduction (dedoping) of the polymer film. The authors also suggested that this intercalated charge dependent conductivity implies that this PANI structure could be used as a memristor [31].

Chiu et al. (2005) utilized elemental analysis to determine the overall doping caused by Iodine $\left(\mathrm{I}_{3}{ }^{-}\right)$and sulfate $\left(\mathrm{SO}_{4}^{-2}\right)$ ions on a PEDOT film and to characterize the conductivity as a function of this overall doping level. This study showed a clear increase in conductivity with the overall doping level and saturation in the conductivity for higher overall doping levels [32].

Furthermore, Zotti (1998) investigated the relationship between the doping level and conductivity of polypyrroles and polythiophenes by using the EQCM technique. The author further explained how this relationship could shed light on the role that polarons and bipolarons have in the conductivity of these polymers. In the case of polythiophenes, 
the conductivity $v s$. doping level (electrons per thiophene ring) had a sigmoidal shape. This relationship started with an increase in conductivity at a specific threshold doping level and reached a plateau at the maximum conductivity. This sigmoidal shape was also obtained for the relationship between conductivity and applied voltage [34].

Also, several studies have used electrochemical doping or dedoping and measured the conductivity of the polymer film as a function of applied voltage between the polymer and the counter electrode. In every case a sigmoidal shape is obtained for this relationship [35] [36] [19] [37].

\subsection{Optical Properties}

Similar to inorganic semiconductors, the features in the optical spectrum of conjugated polymers will depend on the size of the energy bandgap. This optical spectrum drastically changes when the conjugated polymer is doped. The nature of the charge-storage states that emerge upon doping plays a crucial role in determining how the optical spectrum evolves throughout the doping process [38].

As previously mentioned, the doping of a conjugated polymer creates gap states in the form of solitons, polarons and bipolarons. Once these gap states appear there are more than one optical transition and not just the usual photon excitation of an electron from the HOMO (valence) band to the LUMO (conduction) band. In the case of undoped or pristine polyacetylene, the absorbance spectrum shows a broad peak that represents the $\pi$ $\pi^{*}$ electron excitation from the HOMO band to LUMO band with a bandgap of $1.4 \mathrm{eV}$. As the polymer is doped, the amplitude of the peak decreases and another peak emerges at a lower energy of approximately $0.65 \mathrm{eV}$ to $0.75 \mathrm{eV}$. The new peak agrees with the 
appearance of a soliton state at the middle of the bandgap as shown in Figure 3.5(a). This is expected for a degenerate polymer such as polyacetelyne [38].

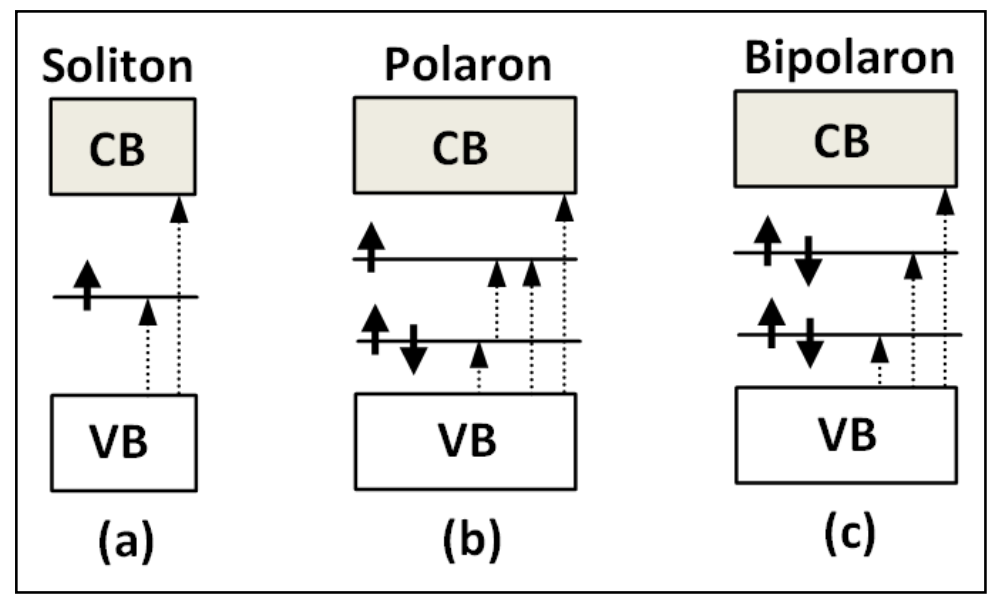

Figure 3.5 Allowed optical transitions [shown by dotted arrows] in the case of (a) a soliton, (b) a polaron and (c) a bipolaron

In the case of a nondegenerate polymer like PEDOT, polaron and bipolaron states are expected to emerge upon doping. In its pristine state, the absorbance spectrum of PEDOT shows a peak at $2.2 \mathrm{eV}$ that is related to the $\pi$ - $\pi^{*}$ electron excitation. As PEDOT is doped, polaron states appear at the bandgap and there are new allowed electron excitations that include transitions from the HOMO band ( $\pi$-band) to both polaron states and a transition between the polaron states. These new possible electron transitions cause additional absorbance peaks to appear in the absorbance spectrum at lower energies (Figure 3.5(b)). Furthermore, these new possible electron transitions become more dominant while the peak associated with the $\pi-\pi^{*}$ electron excitation decreases in amplitude as the polymer is doped. At high levels of doping, polarons can combine to form bipolaron states. However, as shown in Figure 3.5(c), the electron transition between bipolaron states is not allowed because the bipolaron states must be either full 
(negative bipolaron) or empty (positive bipolaron), unlike polaron states [38]. Gustafsson et al. (1994) performed in situ optical absorbance measurements of an electrochemically grown PEDOT:PSS film $\left(\mathrm{ClO}_{4}\right.$ counter anions were also present) as it was doped using an electrochemical cell. They showed that as PEDOT was doped the absorbance peak at $2.2 \mathrm{eV}$ due to the bandgap absorption decreased. Also, they found that two new lower energy $(<0.5 \mathrm{eV}$ and $1.4 \mathrm{eV})$ peaks, due to bipolaron electron transitions, appeared in the absorbance spectra [39]. These electron transitions are illustrated in Figure 3.5(c).

Based on the differences in the optical spectrum of the undoped and doped states of PEDOT, the doped state has a sky blue semi-transparent colour because of its smaller bandgap with absorption energy in the NIR region. The undoped state has a dark blue colour due to a larger bandgap with an absorption energy in the low energy (red) visible light. In the case of PEDOT:PSS, the electrochemical dedoping of this polymer creates a spatial and temporal transition between its two doping states that are characterized by a colour change and observed as dark blue coloration gradient that appear throughout the polymer. This blue coloration is an indirect measurement of the counter-ion (in the case of this work a $\mathrm{Li}^{+}$cation) density given that a higher concentration of the counter-ion causes a higher degree of dedoping, and consequently, a darker blue coloration in the same region. This is the basis of the video analysis performed on the ion migration through the polymer, which is presented in this work. 


\section{Chapter 4}

\section{Conductivity of PEDOT:PSS}

\subsection{Introduction}

The thin film structures used in this work were made from screen-printable inks using a screen printing technique described in reference [19]. A series of PEDOT:PSS devices with length and width variations were fabricated and their conductivity was determined. The profilometer results showed that the size of the polymer device affected the topography of the polymer film and caused the appearance of edge peaks. The conductivity showed a deviation for the larger polymer sample. However, an average conductivity of $975 \mathrm{~S} / \mathrm{m}$ was determined to be adequate for the majority of the polymer devices.

\subsection{Conductivity Measurements}

The inks used to fabricate the polymer devices for this conductivity study were a ERCON silver ink (E-1660-136) and a Orgacon PEDOT:PSS ink (EL-P3040). A graphite ink (E0455-125) was also used in other studies instead of silver ink. To estimate the conductivity of PEDOT:PSS, a design was made in which several two terminal devices of varying width and length were screen printed onto a glass substrate (Figure 4.1). The DC resistance of each device was measured using a Keithley 2400 source-measure unit 
(SMU) and a probing station equipped with a microscope. The length of each device was determined from the mask design that was used to print the PEDOT:PSS layer.

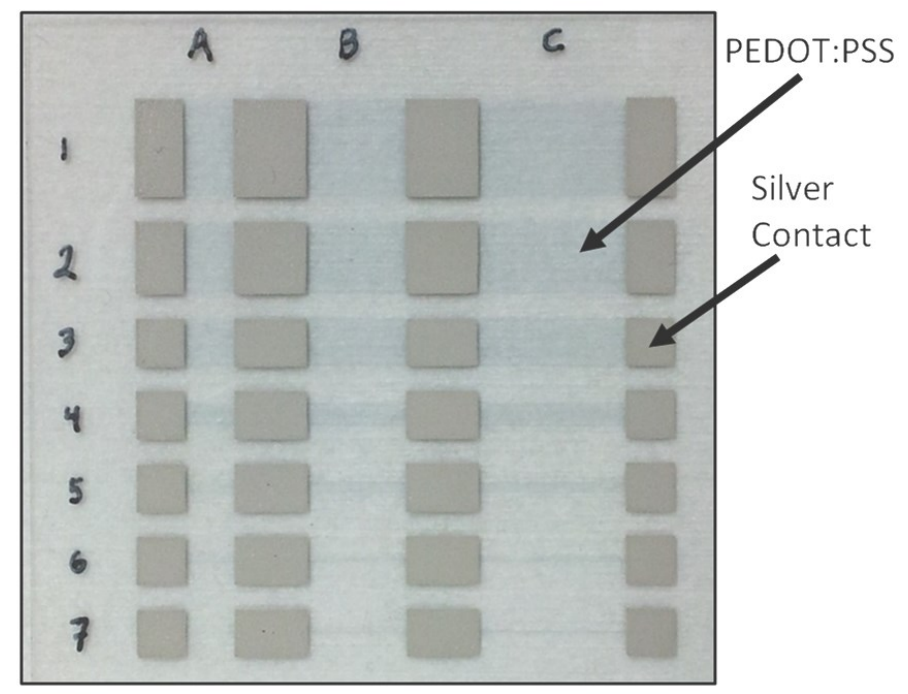

Figure 4.1 Fabricated PEDOT:PSS devices used to measure the polymer conductivity

The cross sectional area of the polymer devices was measured using a Dektak IIA profilometer, which drags a sensitive (low force) stylus along the polymer layer and provides a read out of the height as a function of stylus position. The profilometer can calculate the cross sectional area and has a vertical resolution of about $0.5 \mathrm{~nm}$ and a stylus tip radius of $12.5 \mu \mathrm{m}$. The drying process of a printed polymer film inside an oven will cause thickness variations in the polymer film which are observed in the profilometer scans. In fact a peak feature was observed at both ends of the profilometer scan with a valley in the middle for devices with widths larger than about $1 \mathrm{~mm}$. Also, devices with a width smaller than about $0.4 \mathrm{~mm}$ had a triangular cross section with a single peak in the middle. However, the desired rectangular cross section was approximately obtained for devices with widths of about $0.7 \mathrm{~mm}$. Figure 4.2 shows all three variations previously mentioned. 
(a)

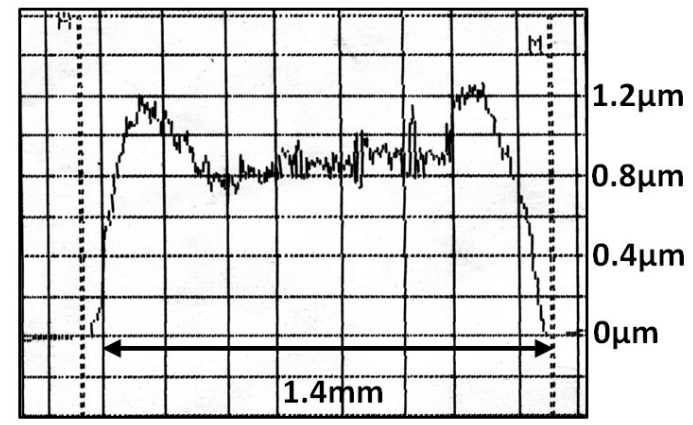

(b)

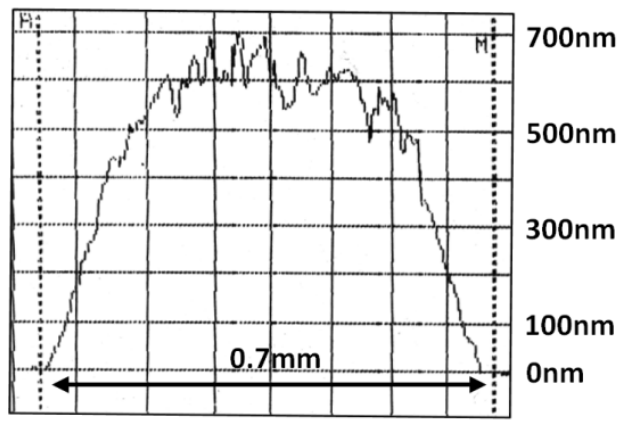

(c)

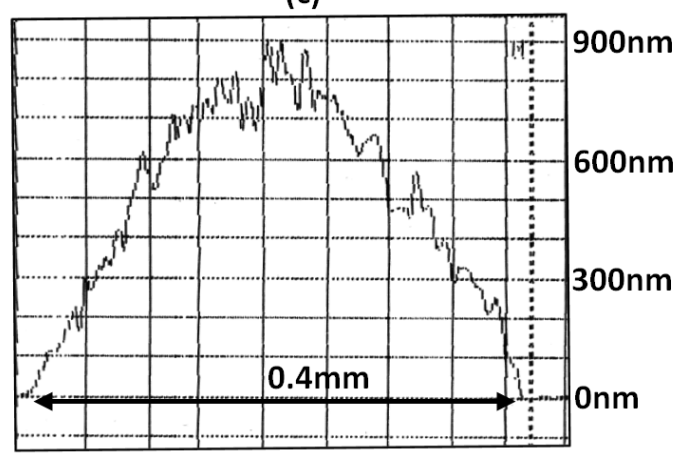

Figure 4.2 Profilometer scans for polymer devices with different widths: (a) $>1 \mathrm{~mm}$, (c) $0.7 \mathrm{~mm}$ and (b) $\leq 0.4 \mathrm{~mm}$.

This thickness variation was taken into account when determining the dimensions of future PEDOT:PSS devices used in this work. The measurements for resistance $(R)$, length $(l)$ and cross sectional area $(A)$ were used to calculate the conductivity $(\sigma)$ of the PEDOT:PSS ink using equation 4.1. Table 4.1 gives the measured and calculated data for the conductivity of PEDOT:PSS where the device ID refers to the array labeling shown in Figure 4.1.

$$
\sigma[S / m]=\frac{l}{R A}
$$


Table 4.1 Conductivity measurements for PEDOT:PSS devices shown in Figure 4.1

\begin{tabular}{|c|c|c|c|c|c|}
\hline $\begin{array}{c}\text { Device ID } \\
\text { Number }\end{array}$ & $\begin{array}{c}\text { Length }(\mathbf{m m}) \\
\pm \mathbf{5 0 \mu \mathrm { m }}\end{array}$ & $\begin{array}{c}\text { Cross Section } \\
\left(\mu \mathrm{m}^{2}\right) \pm 0.5 \mu \mathbf{m}^{2}\end{array}$ & $\begin{array}{c}\text { Resistance } \\
(\mathbf{k} \Omega) \pm 5 \Omega\end{array}$ & $\begin{array}{c}\text { Conductivity } \\
\boldsymbol{\sigma}(\mathbf{S} / \mathbf{m})\end{array}$ & $\begin{array}{c}\text { Error on } \\
\text { Conductivity } \\
\Delta \sigma(\mathbf{S} / \mathbf{m})\end{array}$ \\
\hline A1 & 5.10 & 3793 & 0.96 & 1401 & 16 \\
\hline A2 & 5.10 & 2962 & 1.42 & 1213 & 13 \\
\hline A3 & 5.10 & 2271 & 2.24 & 1003 & 10 \\
\hline A4 & 5.10 & 1243 & 4.14 & 991 & 10 \\
\hline A5 & 5.10 & 556 & 9.48 & 968 & 10 \\
\hline A6 & 5.10 & 301 & 17.66 & 959 & 10 \\
\hline A7 & 5.10 & 227 & 26.93 & 834 & 8 \\
\hline B1 & 10.15 & 3658 & 1.85 & 1500 & 8 \\
\hline B2 & 10.15 & 2865 & 2.72 & 1302 & 7 \\
\hline B3 & 10.15 & 2232 & 4.25 & 1070 & 5 \\
\hline B4 & 10.15 & 1176 & 8.14 & 1060 & 5 \\
\hline B5 & 10.15 & 576 & 18.36 & 960 & 5 \\
\hline B6 & 10.15 & 317 & 33.88 & 945 & 5 \\
\hline B7 & 10.15 & 167 & 61.26 & 992 & 6 \\
\hline C1 & 15.25 & 3806 & 2.87 & 1396 & 5 \\
\hline C2 & 15.25 & 2941 & 4.32 & 1200 & 4 \\
\hline C3 & 15.25 & 2289 & 6.64 & 1003 & 3 \\
\hline C4 & 15.25 & 1210 & 12.91 & 976 & 3 \\
\hline C5 & 15.25 & 551 & 28.00 & 988 & 3 \\
\hline C6 & 15.25 & 328 & 52.16 & 891 & 3 \\
\hline C7 & 15.25 & 204 & 75.92 & 985 & 4 \\
\hline & & & & & \\
\hline
\end{tabular}

Figure 4.3 provides the conductivity values as a function of the cross sectional area of each device. This Figure shows that devices with ID numbers 3-7 (indicated by the dotted rectangle) had a fairly consistent conductivity with a slight downwards trend and an average conductivity of $975 \mathrm{~S} / \mathrm{m}$. Devices with ID numbers 1-2 (indicated by the solid rectangle) had a larger conductivity than $975 \mathrm{~S} / \mathrm{m}$. This conductivity deviation might be attributed to the porosity of the polymer film which seems to lower the apparent conductivity of polymer films with small dimensions. 


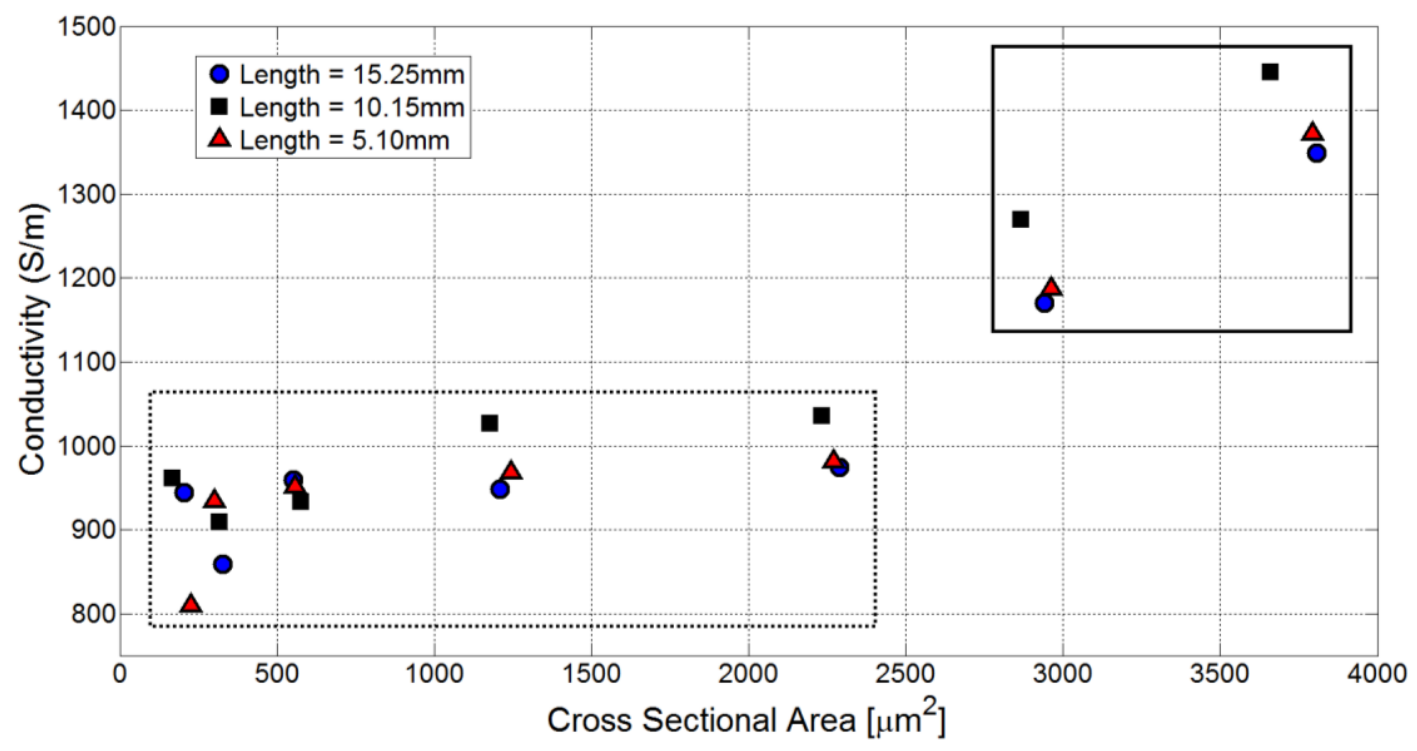

Figure 4.3 Plot of conductivity values for every PEDOT:PSS device shown in Figure 4.1

\subsection{Conclusions}

The conductivity measurements indicated that a screen printed PEDOT:PSS thin film had an average conductivity of $975 \mathrm{~S} / \mathrm{m}$. However, the conductivity deviated from this value as the dimensions of the thin film exceed $1 \mathrm{~mm}$ most likely due to the porosity dependence of the polymer conductivity. An optimal width of $0.7 \mathrm{~mm}$ was determined to give a cross sectional area that was nearly rectangular without any peak edges. 


\section{Chapter 5}

\section{Lithium Diffusion: Pulsed Gradient Spin Echo NMR}

\subsection{Introduction}

Several samples of electrolytes made from lithium perchlorate $\left(\mathrm{LiClO}_{4}\right)$ in propylene carbonate were prepared using different $\mathrm{LiClO}_{4}$ concentrations. The relationship between the $\mathrm{Li}^{+}$ion concentration and its diffusion coefficient in the electrolytes was characterized by a Nuclear Magnetic Resonance technique called Pulsed Gradient Spin Echo (PGSE). The results indicated an inverse linear relationship between the $\mathrm{Li}^{+}$ion concentration and its diffusion coefficient between concentrations of $0.1 \mathrm{M}$ to $1.0 \mathrm{M}$.

\subsection{Lithium Perchlorate Electrolyte Preparation}

A lithium perchlorate $\left(\mathrm{LiClO}_{4}\right)$ electrolyte was prepared by mixing an amount of $\mathrm{LiClO}_{4}$ into the solvent Propylene Carbonate (PC). This solvent has a large dipole moment (polar) and cannot form Hydrogen bonds (aprotic). Furthermore, it has a high dielectric constant of about 65 at room temperature and a boiling point of $242^{\circ} \mathrm{C}$ [12]. Electrolytes with a molarity of $0.1 \mathrm{M}, 0.2 \mathrm{M}, 0.5 \mathrm{M}$, and $1.0 \mathrm{M}$ were made by properly measuring the amount of $\mathrm{LiClO}_{4}$ (molar mass of $106.39 \mathrm{~g} / \mathrm{mol}$ ) and volume of PC needed to make each concentration. Due to the hygroscopic properties of $\mathrm{LiClO}_{4}$, the amount of $\mathrm{LiClO}_{4}$ was weighted on a scale inside a nitrogen filled bag and then poured into a glass bottle. The volume of PC solvent was measured using an Eppendorf Repeater 4780 and 
an Eppendorf Combitip. This was then poured into a glass bottle and the $\mathrm{LiClO}_{4}$ was allowed to dissolve completely.

\subsection{Experimental Setup and Results}

The diffusion coefficient for various concentrations of $\mathrm{LiClO}_{4}$ in $\mathrm{PC}$ electrolytes was measured using the technique called Pulsed Gradient Spin Echo (PGSE) NMR explained in detail in section 2.3.4. The PGSE experiments were performed at the National Research Council (NRC) of Canada using a Bruker DSX400 NMR spectrometer. Computer software was already setup to control the NMR spectrometer and the timing for the RF signals and magnitude of the magnetic field gradients. Each electrolyte sample was poured into a $10 \mathrm{~mm}$ diameter NMR tube that was placed inside the NMR spectrometer. This software was also used to collect and save all the NMR signals and perform the curve fitting on the experimental data to extract the diffusion coefficient for each electrolyte sample. Figure 5.1 shows an example of the experimental data obtained from a PGSE experiment for a $1.0 \mathrm{M}$ electrolyte sample. The curve fitting based on equation 2.25 and calculated by the computer software is also shown in Figure 5.1. The

circle markers in Figure 5.1 represent the normalized intensity of the echo signal for a particular magnetic field gradient strength. 


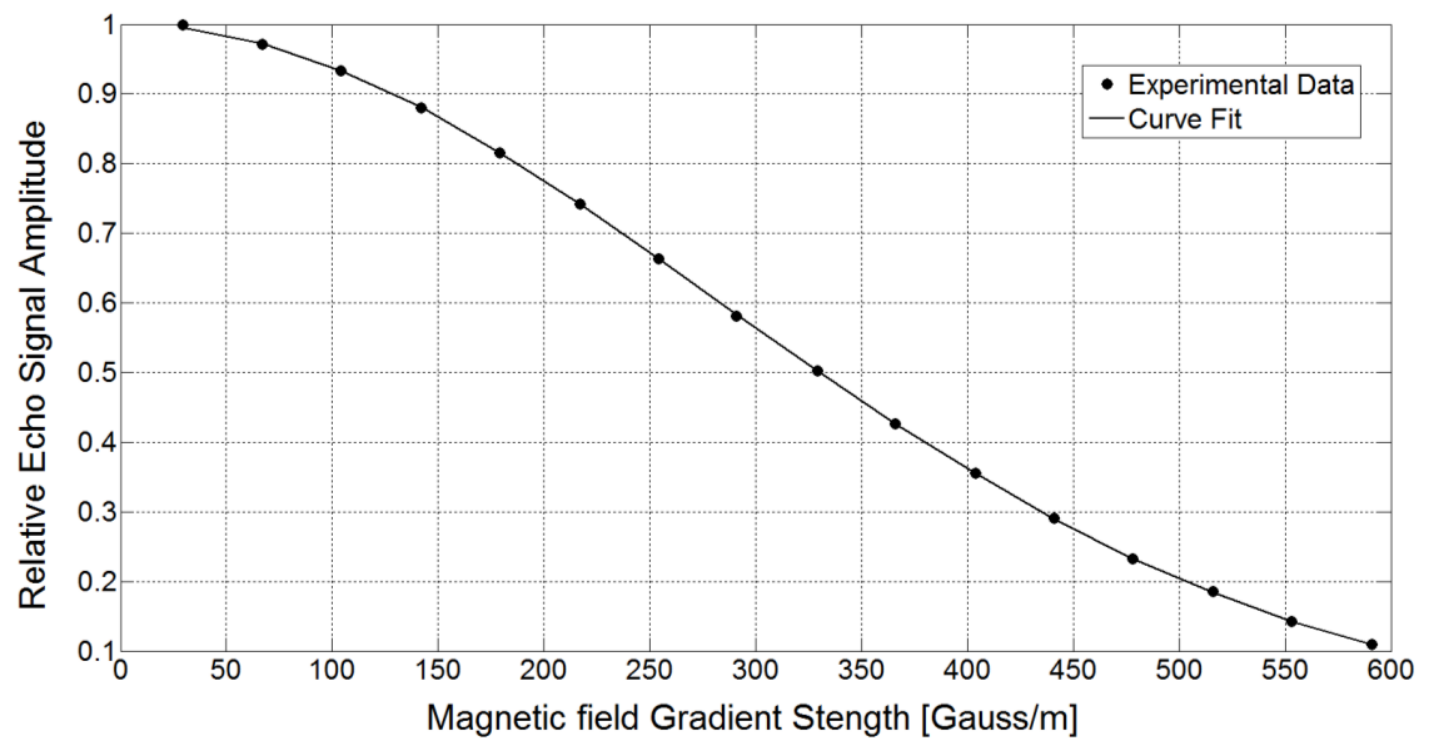

Figure 5.1 Diffusion coefficient calculation using the Stejskal-Tanner curve fitting method

Table 5.1 shows the diffusion coefficients for each of the 4 concentrations of $\mathrm{LiClO}_{4}$ electrolytes. The diffusion coefficient increased as the concentration of $\mathrm{Li}^{+}$ions decreased in the electrolyte. This behaviour is expected since a lower ion concentration means that the ion-ion interaction decreases and the $\mathrm{Li}^{+}$ions do not collide with other ions as frequently. Furthermore, the electrolyte conductivity can be estimated using equation 2.15 and the diffusion coefficient values given in Table 5.1. It is important to remember that equation 2.15 from section 2.2.2 assumes that the larger perchlorate anions $\left(\mathrm{ClO}_{4}^{-}\right)$are less mobile with respect to the smaller $\mathrm{Li}^{+}$cations.

Table 5.1 Experimental data for the diffusion coefficient of $\mathrm{Li}^{+}$ion for various electrolyte concentrations

\begin{tabular}{|c|c|}
\hline $\begin{array}{c}\text { Concentration } \\
\text { (Molar) }\end{array}$ & $\begin{array}{c}\text { Diffusion Coefficient } \\
\left(\mathbf{c m}^{\mathbf{2}} / \mathbf{s}\right) \mathbf{~} \mathbf{1 0}^{-\mathbf{7}}\end{array}$ \\
\hline 1.0 & 7.620 \\
\hline 0.5 & 13.66 \\
\hline 0.2 & 16.87 \\
\hline 0.1 & 18.29 \\
\hline
\end{tabular}


Figure 5.2 shows a plot of the data provided in Table 5.1, which indicates an inverse linear relationship between the ion diffusion coefficient and the ion concentration, as shown by the solid red line. The coefficients for this inverse linear fit are given by equation 5.1. Also, the electrolyte conductivity as a function of $\mathrm{Li}^{+}$ion concentration is given in Figure 5.2, where the blue dashed line was determined using equation 5.1 instead of the values in Table 5.1. The electrolyte conductivity values are comparable to the values given in the literature for similar electrolytes [40].

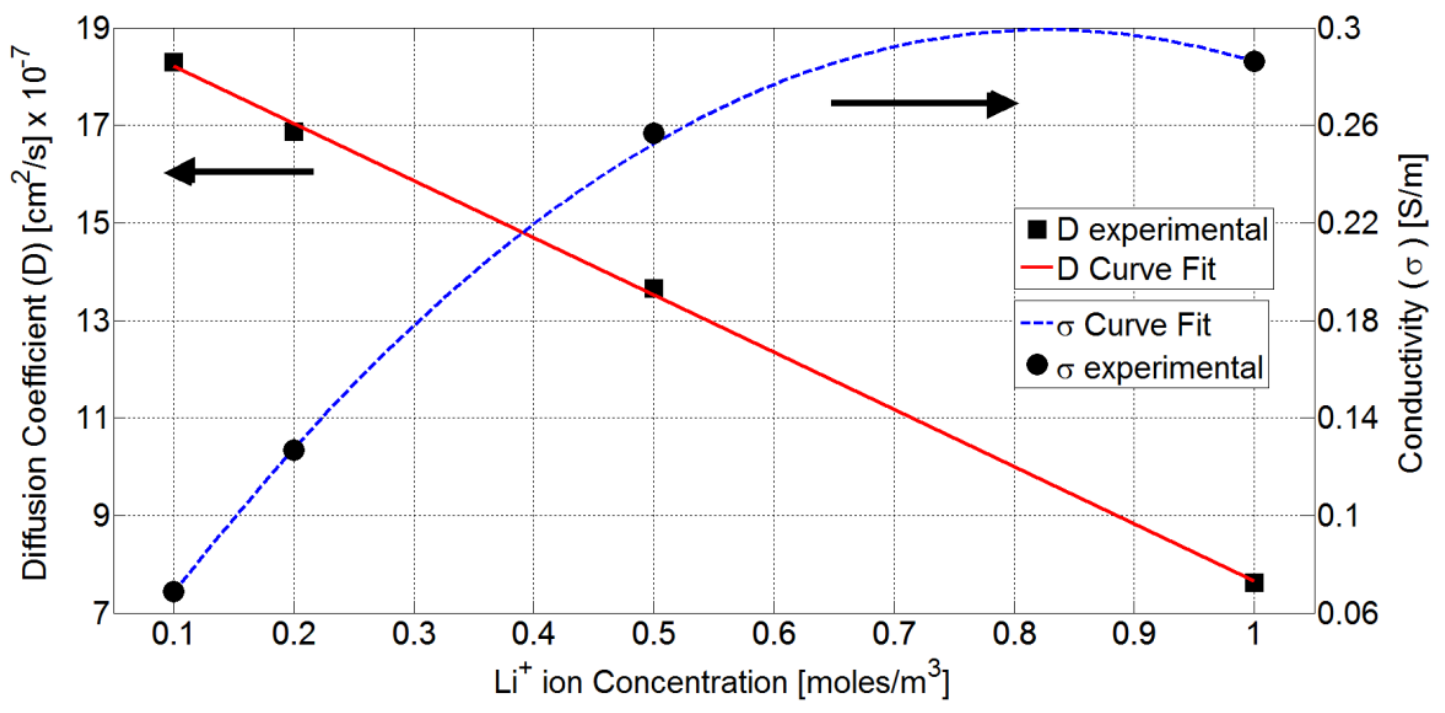

Figure 5.2 $\mathrm{Li}^{+}$ion diffusion coefficient and electrolyte conductivity as a function of concentration of $\mathrm{Li}^{+}$ions in a $\mathrm{LiClO}_{4}-\mathrm{PC}$ electrolyte.

$$
D_{L i^{+}}\left[\frac{c m^{2}}{s}\right]=-\left(11.724 \times 10^{-7}\right) C[M]+19.386 \times 10^{-7}
$$

\subsection{Conclusions}

An inverse linear relationship between the diffusion coefficient of $\mathrm{Li}^{+}$ions and the concentration of these ions in a $\mathrm{LiClO}_{4}$ electrolyte was obtained from the PGSE-NMR 
measurements. These results allow one to calculate the corresponding diffusion coefficient and conductivity for the $\mathrm{LiClO}_{4}$ electrolytes at any concentration between concentrations of $0.1 \mathrm{M}$ to $1.0 \mathrm{M}$. 


\section{Chapter 6}

\section{PEDOT:PSS Conductivity Dependence on Lithium}

\section{Intercalation}

\subsection{Introduction}

An in situ spectroelectrochemical experiment was setup and performed to determine how the conductivity of a thin film of PEDOT:PSS will change during the dedoping process. The PEDOT:PSS device used consisted of a graphite electrode and a PEDOT:PSS electrode enclosed in a sealed volume filled with the $\mathrm{LiClO}_{4}$ electrolyte. An optical setup was used to perform a spectral attenuation spectroscopy experiment, while a source-measure unit (SMU) and a LCR meter were used to dedope the polymer film and to measure its conductivity, respectively. The results showed a sigmoidal shape function for the relationship between the conductivity of PEDOT:PSS and the concentration of intercalated $\mathrm{Li}^{+}$ions. The maximum intercalated $\mathrm{Li}^{+}$ion concentration was determined to be about $1.6 \mathrm{M}$.

\subsection{Device Geometry and Preparation}

The PEDOT:PSS device geometry consisted of a PEDOT:PSS thin film with two metal electrodes on each end which, in analogy to FET devices, were named drain and source. A third electrode, printed from graphite ink and labeled as the gate, was ionically 
connected to the PEDOT:PSS film through the electrolyte. This polymer device will be referred to as polymer electrochemical transistor (PET) device for the rest of the discussion.

The gate, source and drain contacts were made from highly conductive graphite ink instead of a previously used silver ink due to the chemical corrosive effects of silver upon coming into contact with the $\mathrm{LiClO}_{4}$ electrolyte. A short piece of flexible metal wire was attached to each contact by applying a thermal silver (Ag) epoxy. The Ag epoxy was covered with an epoxy resin to avoid possible contact with the electrolyte. Three pieces of Kapton tape were taped together, hole-punched and placed over the polymer film. A few drops of the $\mathrm{LiClO}_{4}$ electrolyte were applied to the hole-punched circular area and then a square piece of glass was positioned on top of the electrolyte. The electrolyte concentration employed was 1 millimolar $(\mathrm{mM})$. The Kapton tape layers and the piece of glass created a volume filled with electrolyte. The edges of the piece of glass were dabbed with Norland UV adhesive and exposed to UV light for 10 minutes to seal the device and avoid electrolyte leakage. Figure 6.1 and 6.2 show a picture of a device before and after the sealing process, respectively. 


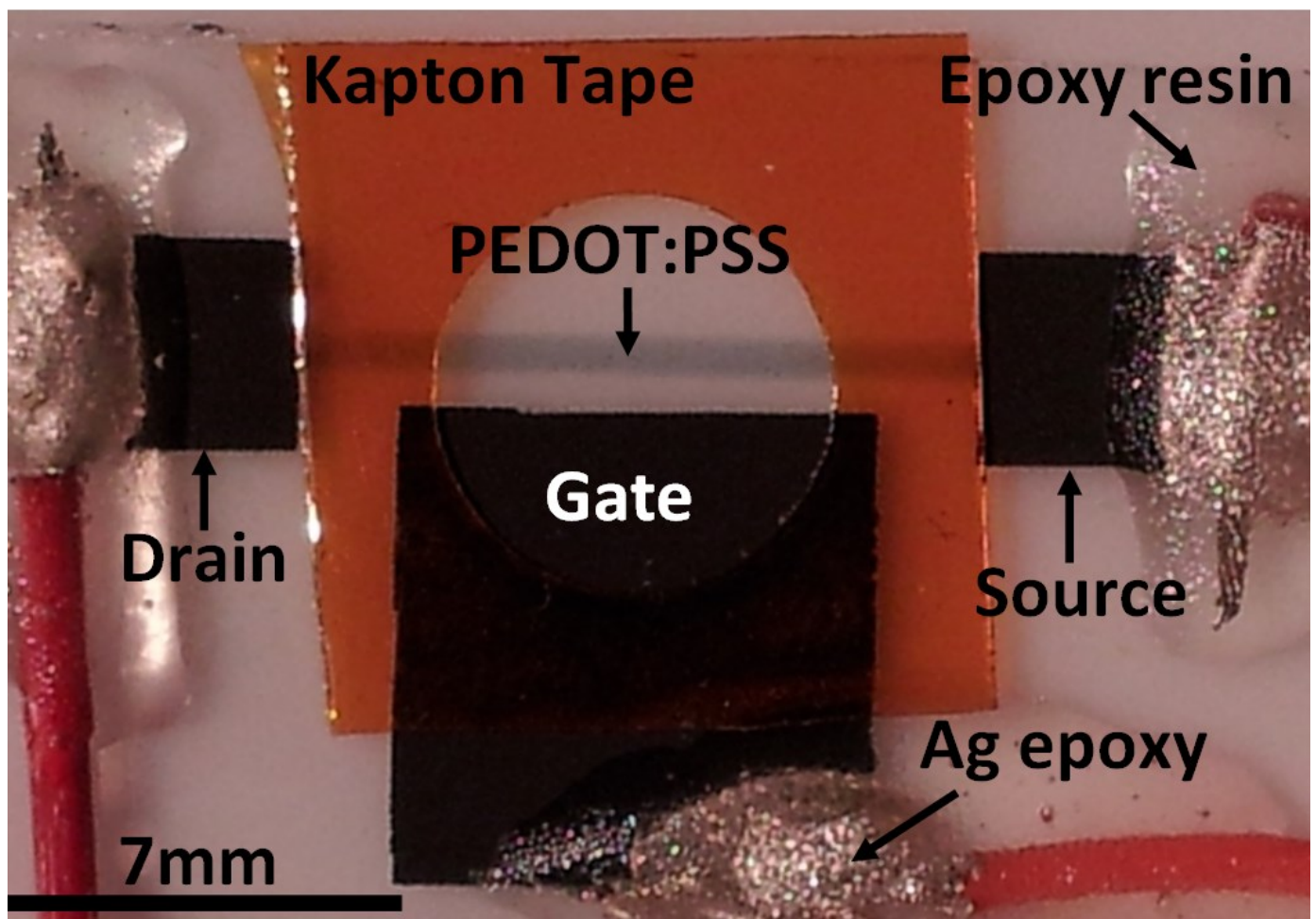

Figure 6.1 PET device with wires attached by silver epoxy and before it was sealed

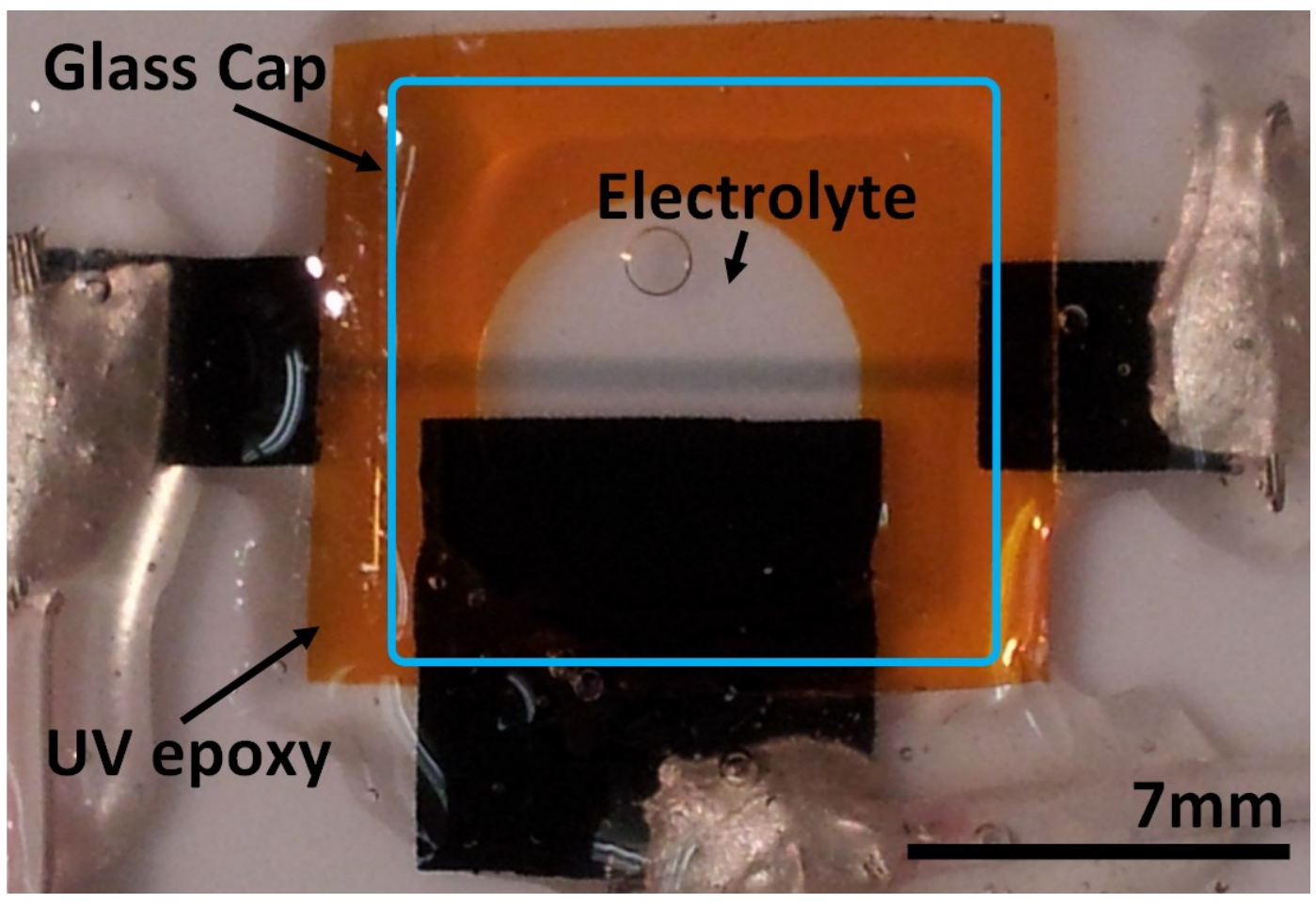

Figure 6.2 PET device after it was completely sealed 
Since the three Kapton tape layers had a thickness of $0.2 \mathrm{~mm}$ and the diameter of the hole-punched circle was $7 \mathrm{~mm}$, the total electrolyte volume was about $7.7 \mathrm{~mm}^{3}$. The width of the polymer film was chosen based on profilometer measurements (refer to section 4.2), which showed that a width of about $0.7 \mathrm{~mm}$ gave almost a rectangular cross section. Due to this narrow polymer width, a number of polymer layers were printed on top of each other in order to increase the polymer thickness and obtain a low resistance of a few thousand Ohms. Overall, the PEDOT:PSS film had a width and length of $0.7 \mathrm{~mm}$ and $12 \mathrm{~mm}$, respectively, and a thickness of $1.6 \mu \mathrm{m}$. However, the effective length of the PET device is $7 \mathrm{~mm}$ since this is actual length of polymer that will come into contact with the electrolyte, as shown in Figure 6.2. Therefore, the volume of active PEDOT:PSS was $7.84 \times 10^{-6} \mathrm{~cm}^{3}$. The $7 \mathrm{~mm}$ section of polymer will be referred to as the active region.

\subsection{Calculation of Redox Sites Density}

Following the calculation provided elsewhere [7], the density of available redox sites is determined using the dimension of the PEDOT:PSS film used in the PET device. The EL-P3040 PEDOT:PSS ink had a ratio of PEDOT to PSS of 1 to 2.5 and a density ( $\rho_{\text {PEDOT:PSS }}$ of $1.01 \mathrm{~g} / \mathrm{cm}^{3}\left(\right.$ at $20^{\circ} \mathrm{C}$ ) as provided by the manufacturer. Also, the molecular weight $(M W)$ of monomeric units of PEDOT and PSS was $140 \mathrm{~g} / \mathrm{mol}$ and $182 \mathrm{~g} / \mathrm{mol}$, respectively. Therefore, the molecular weight of the PEDOT:PSS complex was 595

$\mathrm{g} / \mathrm{mol}$, which gives a molar density for EDOT units equal to $595 \mathrm{~mol} / \mathrm{cm}^{3}$. Finally, the density of EDOT units $\left(\rho_{E D O T}\right)$ available for a redox reaction was calculated to be $1.02 \times 10^{21}$ (units $/ \mathrm{cm}^{3}$ ). The complete calculation is shown below. 


$$
\begin{gathered}
M W_{\text {PEDOT:PSS }}=M W_{\text {PEDOT }}+2.5\left(M W_{\text {PSS }}\right)=140\left[\frac{\mathrm{g}}{\mathrm{mol}}\right]+2.5\left(182\left[\frac{\mathrm{g}}{\mathrm{mol}}\right]\right)=595\left[\frac{\mathrm{g}}{\mathrm{mol}}\right] \\
\rho_{\text {EDOT }}=\frac{N_{A}\left(\rho_{\text {PEDOT:PSS }}\right)}{\left(M W_{\text {PEDOT:PSS }}\right)}=\frac{6.0221 \times 10^{23}\left[\frac{\text { units }}{\mathrm{mol}}\right]\left(1.01\left[\frac{\mathrm{g}}{\mathrm{cm}^{3}}\right]\right)}{\left(595\left[\frac{\mathrm{g}}{\mathrm{mol}}\right]\right)} \cong 1.02 \times 10^{21}\left[\frac{\text { units }}{\mathrm{cm}^{3}}\right] \\
\rho_{\text {redox }}=\rho_{\text {holes }}=\frac{\rho_{\text {EDOT }}}{3\left[\frac{\text { units }}{\text { hole }}\right]}=\frac{1.02 \times 10^{21}\left[\frac{\text { units }}{\mathrm{cm}^{3}}\right]}{3\left[\frac{\text { units }}{\text { hole }}\right]} \cong 3.4 \times 10^{20}\left[\frac{\mathrm{holes}}{\mathrm{cm}^{3}}\right]
\end{gathered}
$$

According to electrochemical measurements [7], it is assumed that only about a third of $1.02 \times 10^{21}$ EDOT monomers per $\mathrm{cm}^{3}$ would participate in the redox reaction. Therefore, the density of holes $\left(\rho_{\text {holes }}\right)$ introduced into the PEDOT:PSS complex after it has been completely $p$-doped was $3.4 \times 10^{20}$ holes per $\mathrm{cm}^{3}$. Hence, the density of available redox sites $\left(\rho_{\text {redox }}\right)$ in a PEDOT:PSS film was given by the same value of $3.4 \times 10^{20}$ sites $/ \mathrm{cm}^{3}$. In order to estimate the total number of available redox sites, this redox site density was multiplied by the volume of PEDOT:PSS which was $7.84 \times 10^{-6} \mathrm{~cm}^{3}$. Therefore, one would expect that the electrochemical experiments would give a value for the total charge intercalated into the PEDOT:PSS film that is within $2.67 \times 10^{15}$ electrons or $427 \mu \mathrm{C}$. Moreover, the electrolyte must have an appropriate concentration to supply enough ions so that the resistance of the polymer film can reach a saturation state where all the redox sites are dedoped. This concentration $\left(n_{x}\right)$ was calculated to be at least $0.6 \mathrm{mM}$ (millimoles/litre) for the electrolyte volume of $7.7 \mathrm{~mm}^{3}$ that was used in the PET devices.

$$
n_{x}=\frac{2.67 \times 10^{15}[\text { ions }]}{\left(V \cdot N_{A}\right)}=\frac{2.67 \times 10^{15}[\text { ions }]}{\left(7.7 \times 10^{-9}\left[\mathrm{~m}^{3}\right] \cdot 6.0221 \times 10^{23}\left[\frac{\text { ions }}{\mathrm{mol}}\right]\right)} \cong 0.576 \mathrm{mM}
$$

Although a concentration several orders of magnitude higher could also be used, preliminary experiments showed that high concentrations such as $1.0 \mathrm{M}$ were prone to 
cause undesired side reactions resulting in degradation of the polymer. Therefore, a concentration of $1 \mathrm{mM}$ was chosen for all experiments discussed in this chapter.

\subsection{Spectral Attenuation Spectroscopy Setup}

A 100Watt quartz tungsten halogen (QTH) lamp was used as a light source of visible (VIS) and near infrared (NIR) light. This QTH lamp was housed inside a Newport monochromator illuminator (model: 7340) and a constant current of $8.34 \mathrm{~A}$ at $12 \mathrm{VDC}$ was supplied to the lamp by an Oriel radiometric power supply (model: 68931). A Newport Cornerstone 130 motorized 1/8m monochromator (model: 74000) with two Cornerstone ruled diffraction gratings installed inside its housing was utilized to select and shine a very narrow range of wavelengths onto the PEDOT:PSS film from the broad VIS-NIR light source spectrum. The monochromator accomplished this by rotating the selected grating and was connected to the computer through a GPIB interface. The output light from the monochromator is quasi-monochromatic with a spectral width of less than 10nm. The first ruled grating (model: 74028), with 600 lines $/ \mathrm{mm}$ and a $1 \mu \mathrm{m}$ blazed wavelength, provided a high efficiency in the NIR region while the second grating (model: 74024), with 1200 lines/mm and a 350nm blazed wavelength, provided a high efficiency in the visible spectrum. These two gratings allowed a total optical spectrum from $200 \mathrm{~nm}$ to $2.5 \mu \mathrm{m}$. Usually the first order diffraction wavelength is desired in an experiment, however, based on the grating equation shown in equation 6.1 , one can see that at a fixed position of the grating there are higher order $(|m|>1)$ wavelengths $(\lambda)$ that are shorter than the first order $(m= \pm 1)$ wavelength. This would cause overlap between the orders. In equation $6.1, a$ is the period of the grating, $\lambda$ is the wavelength of light, $m$ is the 
order of diffraction, and $\theta_{\mathrm{i}}$ and $\theta_{\mathrm{i}}$ are the angle of incidence and the angle for a maxima in the diffraction pattern, respectively.

$$
\lambda=\frac{a\left(\sin \theta_{i}+\sin \theta_{m}\right)}{m}
$$

A Thorlabs motorized precision rotation mount (model: PRM1Z8) was used to rotate a vertical post with two horizontally oriented longpass filters to remove these unwanted higher order wavelengths. The two longpass filters (models: FEL0600 and FEL1100) blocked all wavelengths below $600 \mathrm{~nm}$ and $1100 \mathrm{~nm}$ and were used at wavelengths equal to or greater than $650 \mathrm{~nm}$ and $1150 \mathrm{nmk}$, respectively. A circular shutter and two lenses were used to focus the light onto the PEDOT:PSS sample, which was placed onto a sample holder that could move in the $x, y$ and $z$ directions by means of three manual micrometer driven linear translation stages. Since the focus beam was wider than the width of the PEDOT:PSS film and the beam would slightly move up or down when the diffraction grating was changed, a blocking aperture with a gap narrower than the PEDOT:PSS width was used so that only the light that passed through the aperture and the PEDOT:PSS film would be considered in the optical measurements. After passing through the sample, the beam was split into two beams by a beamsplitter and more lenses were used to redirect each light beam into a Thorlabs standard photodiode power sensor. The first power sensor (model: S120C) was a Silicon (Si) photodiode with a wavelength range of $400 \mathrm{~nm}$ to $1100 \mathrm{~nm}$ and a resolution of $1 \mathrm{nW}$, while the second power sensor (model: S122C) was a Germanium (Ge) photodiode with a wavelength range of 700nm to $1800 \mathrm{~nm}$ and a resolution of $2 \mathrm{nW}$. However, the Ge photodiode had a larger dark power ( $\sim$ between $0.5 \mu \mathrm{W}$ and $50 \mathrm{nW}$ ) than the Si photodiode ( $\sim$ between $40 \mathrm{nW}$ and $5 \mathrm{nW})$ due to 
the smaller bandgap of Ge. As a result, a higher intensity light had to be used for the Ge photodiode in order to increase the optical signal to noise ratio. Each photodiode sensor was connected to a Thorlabs optical power and energy meter (model: PM100USB) which was connected to the computer via a USB interface. Figure 6.3 shows the optical setup with all of its previously mentioned components.

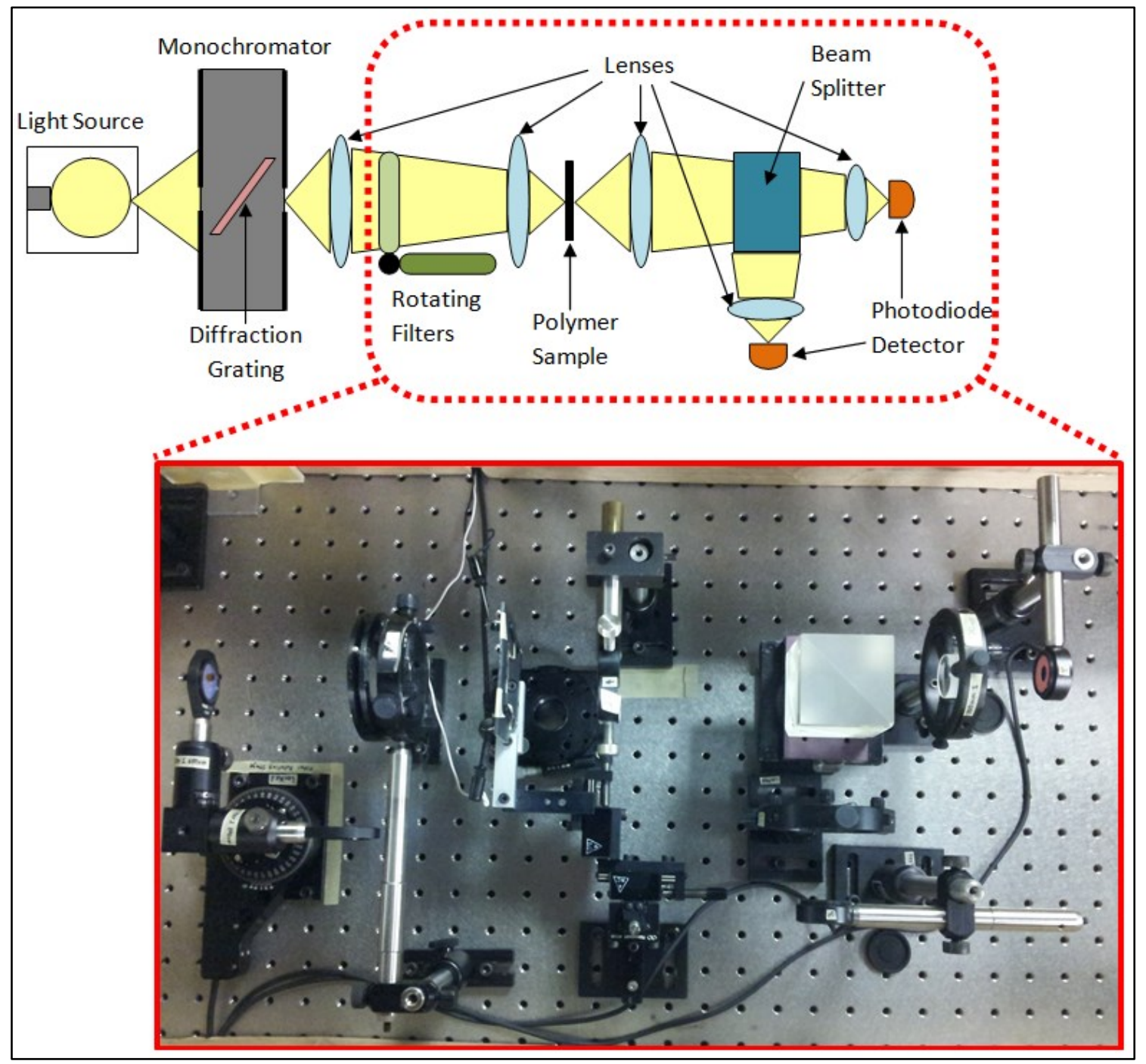

Figure 6.3 Diagram of the optical setup used in the spectroelectrochemical experiments 
In order to calculate the spectral attenuation (SA) of the polymer device as a function of wavelength, a reference spectral measurement $\left(\mathrm{SM}_{\mathrm{Ref}}\right)$ was done by removing the assembled polymer sample from the path of the incident light beam and then measuring the optical power as a function of wavelength. Then the sample was placed back in the optical set and the previous power measurement was repeated in order to obtain the sample spectral measurement $\left(\mathrm{SM}_{\text {Sample }}\right)$. The difference between the $\mathrm{SM}_{\mathrm{Ref}}$ and the $\mathrm{SM}_{\text {Sample }}$ is considered to be the amount of power blocked by the polymer device as a function of wavelength. Therefore, the SA of the polymer device as a function of wavelength was calculated by ratio of the amount of power blocked by the polymer device and the $\mathrm{SM}_{\mathrm{Ref}}$, as shown in equation 6.2.

$$
S A=\frac{S M_{\text {Ref }}-S M_{\text {Sample }}}{S M_{\text {Ref }}}
$$

\subsection{Electrochemical Setup}

A Keithley 236 SMU supplies a voltage and measures the electrochemical current during the dedoping process of the PEDOT:PSS film. When a voltage was applied to the PET device the gate potential was raised or lowered with respect to the drain and source potential. Hence, the positive lead from the SMU was connected to the gate contact of the PET device and then the source and drain contacts were both connected to ground. This means that an applied positive voltage dedopes the PEDOT:PSS film since the $\mathrm{Li}^{+}$ions go towards ground and intercalate into the polymer film. Connecting both the drain and source to ground ensures that the PEDOT:PSS film is dedoped uniformly. The SMU also 
measured the electrochemical current due to the dedoping of PEDOT:PSS and it was connected to the computer through a GPIB interface.

A Hewlett Packard multi-frequency LCR meter (model: 4274A) applied an AC signal and measured the resultant voltage and current across its two leads to measure the impedance of the sample. This LCR meter was connected between the drain and source electrodes to measure the resistance of the PEDOT:PSS film. To avoid measurement issues caused by having the SMU and LCR meter connected to the PET device at the same time, a Keithley 705 scanner was setup to switch the connections so that only one instrument was connected to the device at a time. The 705 scanner used a switching card that allowed matrix switching of its inputs to the outputs by means of a relay matrix. Therefore, the three terminals of the PET device, the LCR meter and the SMU were connected to a Keithley 7052 4x5 matrix switching card which was controlled by the 705 scanner. The LCR meter, SMU and scanner were all connected to the computer via a GPIB interface. Figure 6.4 illustrates the connections made between the PET device, LCR meter, SMU and scanner and it also shows a schematic of the matrix switching card connections, where a black circle represents a switchable connection between a row and a column. 


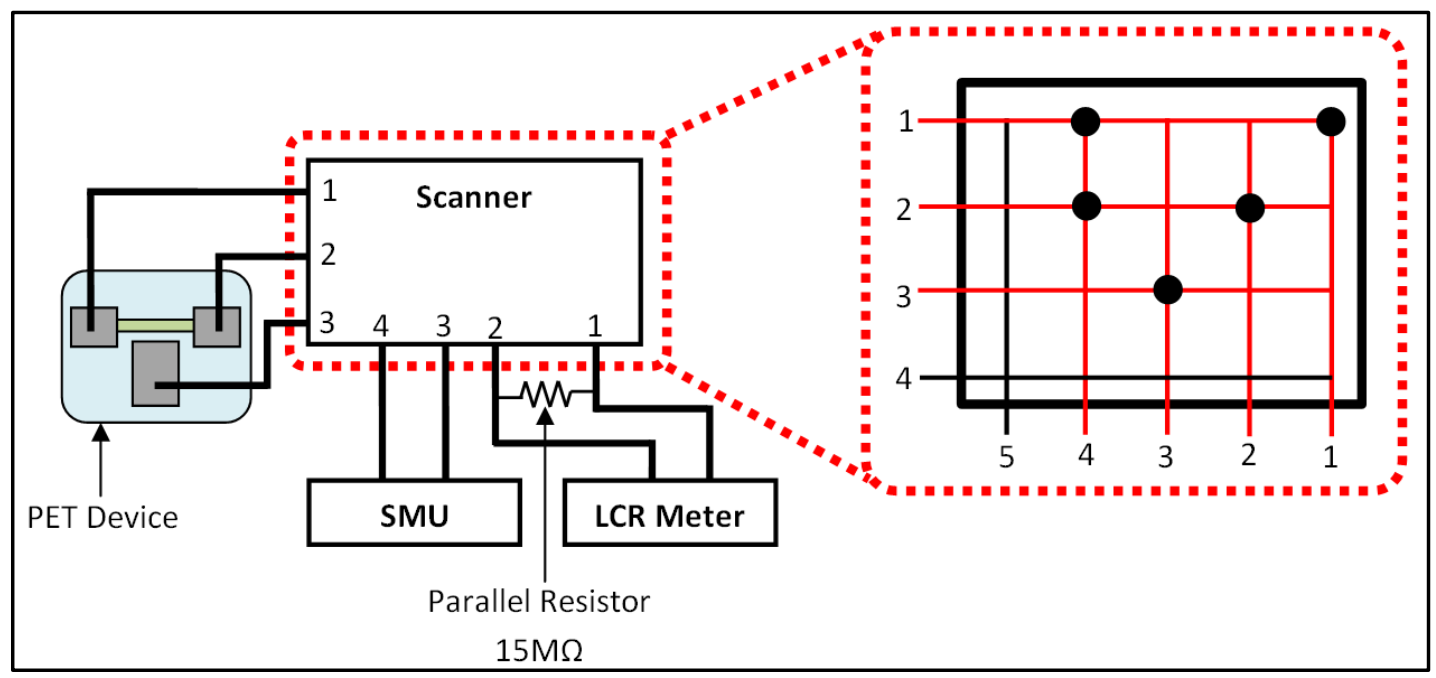

Figure 6.4 Connections among the PET device, SMU, LCR meter and Scanner including the connections inside the $4 \times 5$ matrix switching card

\subsection{Spectroelectrochemical Experiment Control Program}

The entire spectroelectrochemical experiment was automated with the help of Hewlett-Packard Visual Engineering Environment (HP VEE) software. This software enables the user to send and receive data from an instrument through an USB or GPIB interface. A VEE program was written to provide setup parameters to each instrument, perform sequential tasks, and collect and save the experimental data. The program structure began with initialization of instruments. Next, the dark power measurement for each photodiode sensor was recorded and an optical calibration was performed by measuring the optical spectrum of the QTH light source. This was then used to calculate the spectral attenuation of the PEDOT:PSS film. The PEDOT:PSS sample was placed in the optical setup and connected to three alligator clips which were connected to the 705 scanner. The PEDOT:PSS optical spectrum was measured and the spectral attenuation was calculated. Once the spectral attenuation of the polymer gave the expected result for a doped state of the polymer, the program was used to make automatic measurements. 


\subsubsection{Type of Programs}

Preliminary experiments of iterative dedoping and doping of a PEDOT:PSS film showed that the change in the polymer film resistance would evolve differently for each iteration. Eventually, the behaviour for the resistance change of the polymer converges to a consistent behaviour only after the dedoping and doping process was repeated several times. For this reason, a cycling program was written to apply a staircase voltage waveform to the PET device and allow an iterative dedoping and doping process to occur. This voltage waveform would cycle the device between its two doping states until the transition between the two states would converge onto a single shape and hence this ensured that the behaviour of the PET device was stable and ready for further analysis. Following the PET device cycling, the spectroelectrochemical experiment was performed.

In the spectroelectrochemical experiment, a staircase voltage waveform was applied to the PEDOT:PSS device starting from $-0.1 \mathrm{~V}$ up to $2 \mathrm{~V}$ and then back down to $-0.1 \mathrm{~V}$. The voltage step was $0.1 \mathrm{~V}$ and the step duration was 20 minutes. The experiment began by measuring the resistance of the film before any voltage was applied; this was followed by the application of a constant voltage to the PET device. During the constant voltage step, the electrochemical current and the optical power at a fixed wavelength were measured by the SMU and the power meters, respectively. Every 2 minutes the resistance of the PEDOT:PSS film was sampled by the LCR meter. Once the step duration was completed, the current, optical and resistance measurements were saved and then a complete optical spectrum was measured from $400 \mathrm{~nm}$ to $1800 \mathrm{~nm}$. This constitutes all the measurements performed during a voltage step, which were repeated for the next voltage 
step until the final voltage was reached. Then the voltage decreased in the same manner back to $-0.1 \mathrm{~V}$. The vast amount of data was saved into well-organized files that were analyzed using a MatLab program which allowed for quick data manipulation and calculations.

\subsection{Experimental Result}

\subsubsection{Preliminary Cycling}

The experimental results for a PET device filled with a $1 \mathrm{mM}$ electrolyte concentration and having a PEDOT:PSS film with an initial resistance of $11.16 \mathrm{k} \Omega$ will be discussed below. Upon application of the electrolyte the resistance of the polymer film increased slightly to $12.1 \mathrm{k} \Omega$. All resistances were measured with the LCR meter set to $40 \mathrm{mV}$ and a frequency of $10 \mathrm{kHz}$. The low AC signal amplitude guaranteed that no electrochemical reaction would take place while the $10 \mathrm{kHz}$ frequency further ensured that $\mathrm{Li}^{+}$ions could not drift by any significant amount and hence the LCR meter measuring signal did not significantly disturb the state of the polymer film. As previously mentioned in section 6.6.1, the prepared PET device was cycled prior to performing the spectroelectrochemical experiment. The cycling was performed using a staircase voltage waveform with a minimum of $-0.5 \mathrm{~V}$ and maximum of $1.5 \mathrm{~V}$. As a reminder, a negative and positive voltage causes the doping (oxidization) and dedoping (reduction) of the polymer film in the PET device, respectively. A higher negative voltage was not used since previous experiments had shown that the PET devices could be overoxidized and result in a damaged polymer film, which has also been reported in the literature [23]. The cycling was done for an hour and each $0.2 \mathrm{~V}$ step lasted 20 seconds. Figure 6.5 shows the 
resistance of the film as a function of the applied voltage for 10 cycles and the inset shows the change in spectral attenuation of the polymer at $625 \mathrm{~nm}$ as a function of applied voltage. The black arrows represent the progression from the first to last cycle.

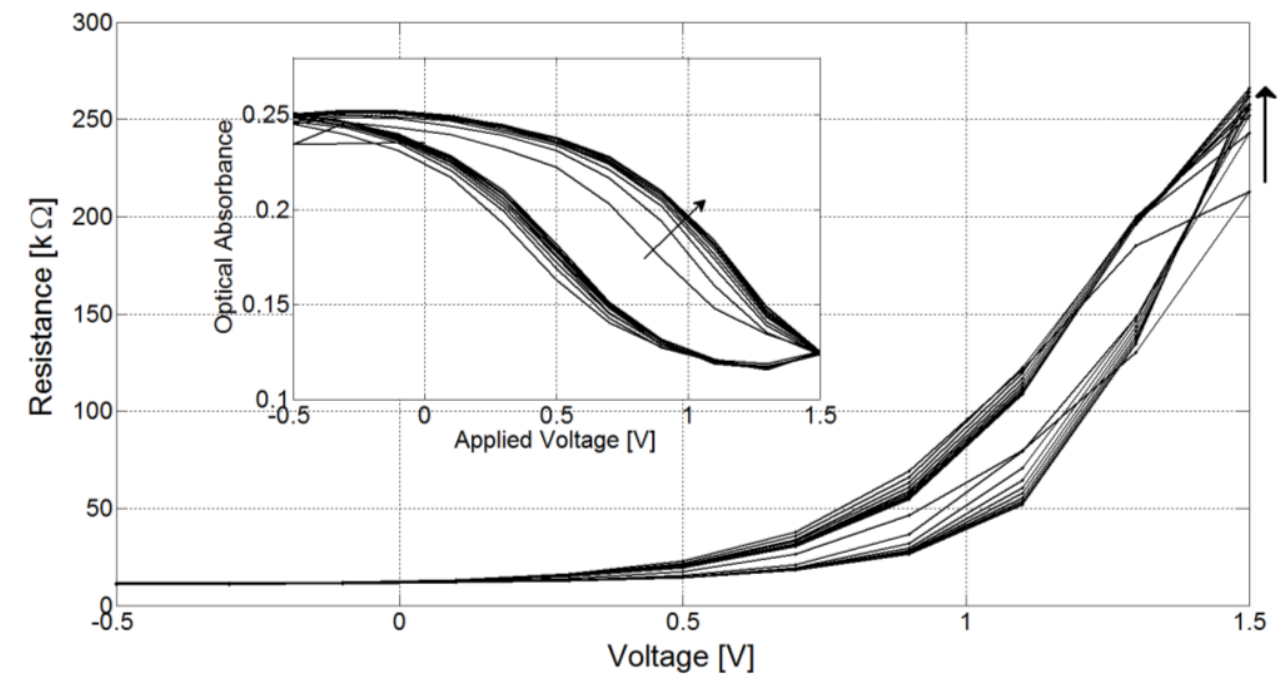

Figure 6.5 Resistance and spectral attenuation at $625 \mathrm{~nm}$ for a PET device as a function of voltage during cycling

It is clear from the Figure above that the PET device converged towards consistent behaviour. During the cycling, the PET device had a minimum and maximum resistance of $12.1 \mathrm{k} \Omega$ and $266 \mathrm{k} \Omega$ which gave an on/off ratio of about 22 . It is also important to highlight the fact that the polymer film experienced no degradation during the cycling since its resistance had an insignificant change after completing the cycling. This is only possible by restricting the applied voltage to values lower than $\pm 2.0 \mathrm{~V}$.

\subsubsection{Resistive Measurements}

The optical and resistive measurements performed during the spectroelectrochemical experiment showed that the PET device reached a highly dedoped state. Figure 6.6 shows 
the measurements for the resistance of the polymer film as a function of voltage during the stepping up (blue) and stepping down (red) voltage phases.

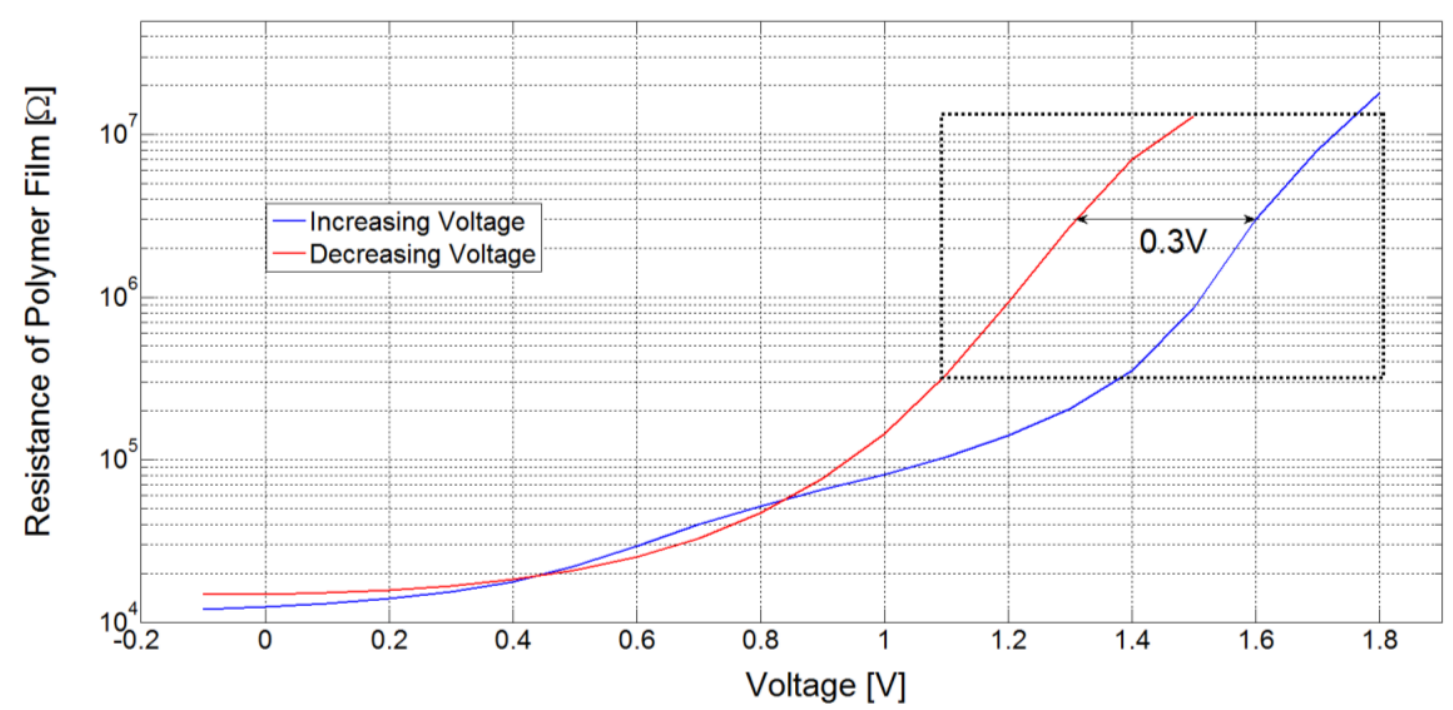

Figure 6.6 Resistance vs. applied voltage for a PET device during the spectroelectrochemical experiment

Although the voltage waveform increased to $2.0 \mathrm{~V}$, the resistance reached by the polymer film beyond $1.8 \mathrm{~V}$ was outside of the measurable range of the LCR meter (> $20 \mathrm{M} \Omega$ ). Hence, there was no data collected until the voltage applied decreased to $1.5 \mathrm{~V}$ causing the polymer resistance to fall below the $20 \mathrm{M} \Omega$. One can see in Figure 6.6 that a total resistance change of 3 orders in magnitude was measured and that the shape for increasing and decreasing resistance processes behaved very similarly. However, it is clear that there was a constant left shift in voltage of roughly $-0.3 \mathrm{~V}$ within the resistance range of $13 \mathrm{M} \Omega$ and $300 \mathrm{k} \Omega$ as shown by the dotted black box in Figure 6.6. This shift vanished below $300 \mathrm{k} \Omega$ and the two curves converged into one. This hysteresis-like behaviour has been reported in the literature [23] and will be considered in subsequent spectroelectrochemical experiments. 
In light of the resistive measurements limitations encountered with the LCR meter, the following experiments were performed with a $15 \mathrm{M} \Omega$ resistor connected in parallel to the drain and source contacts of the PET device to maintain the device resistance below $20 \mathrm{M} \Omega$. The actual polymer film resistance $\left(R_{p}\right)$ was calculated using equation 6.3 where $R_{L C R}$ represents the resistance measurement from the LCR meter.

$$
R_{p}=\frac{(15 M \Omega) R_{L C R}}{15 M \Omega-R_{L C R}}
$$

Three more experiments were performed to determine the resistance saturation value and to address the resistive hysteresis-like behaviour previously observed. The only variation during these three experiments was a shorter voltage step of 8 minutes, instead of 20 minutes, and different maximum voltages of $1.0 \mathrm{~V}, 1.5 \mathrm{~V}$ and $2.5 \mathrm{~V}$. The resistance change obtained for each experiment is shown in Figure 6.7. It is worth noting that in each measurement cycle the rightmost curve corresponds to an increasing voltage while the leftmost curve corresponds to a decreasing voltage, which is indicated by the black arrows. 


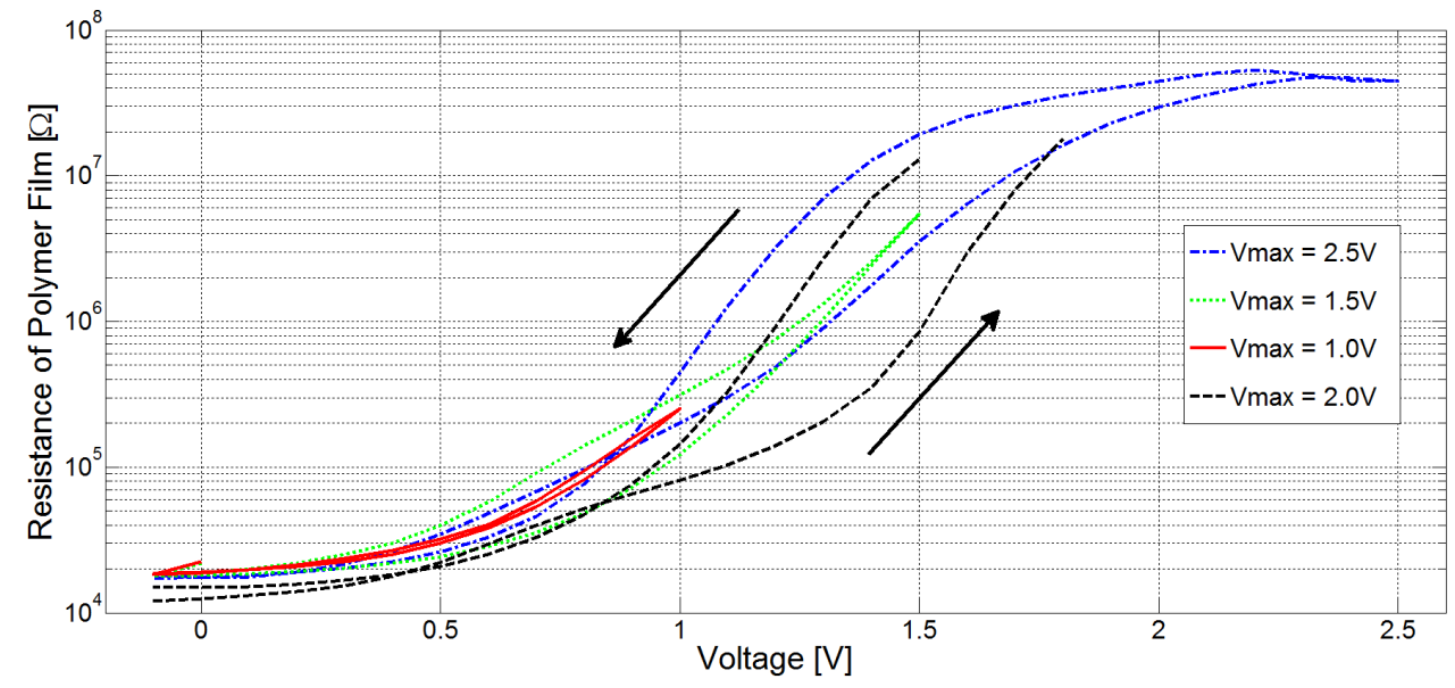

Figure 6.7 Resistance vs. applied voltage for a PET device during the reduction of the polymer film and for different maximum applied voltages

One can see that the previously mentioned left shift in voltage or hysteresis-like behaviour applied to all the curves. However, as the maximum voltage decreased the magnitude of the voltage shift also decreased. In the case of a maximum voltage of $1.0 \mathrm{~V}$, the hysteresis almost vanished completely creating a single-valued relationship between the applied voltage and resistance of the polymer film. The most important curve in Figure 6.7 corresponds to the case of a maximum voltage of $2.5 \mathrm{~V}$, which showed a complete transition between a state of low resistance (doped) of $\sim 17 \mathrm{k} \Omega$ and high resistance (dedoped) of $45 \mathrm{M} \Omega$ including the crucial resistance saturation at about $2.2 \mathrm{~V}$ and an on/off ratio of $\sim 2500$. Similar on/off ratios have been reported in the literature for structures similar to the PET device used in this work [21]. Although the PET device provided reproducible results, as demonstrated by the similarity among the curves in Figure 6.7, a slight increase in the initial resistance of the polymer film was observed every time the PET device was tested again. This was a sign that driving the voltage close 
to $2.0 \mathrm{~V}$ began a degradation of the polymer, which can ultimately render the polymer nonconductive. This was caused by the appearance of possible side reactions, besides the desired redox reaction, which can over-reduce the polymer, change its chemical structure or create morphological modifications which have been reported to greatly affect the conductivity of the polymer [28].

In order to capture the resistive behaviour of the polymer for negative applied voltages (oxidation), another experiment was performed in which the voltage was decreased from $0 \mathrm{~V}$ to $-2.5 \mathrm{~V}$ and back to $0 \mathrm{~V}$, with all other parameters the same as previously described. The initial resistance of the device was $20 \mathrm{k} \Omega$. Figure 6.8 shows the resistance of the polymer $v s$. applied voltage for $0 \mathrm{~V}$ to $-0.5 \mathrm{~V}$, while the Figure inset shows the entire voltage range.

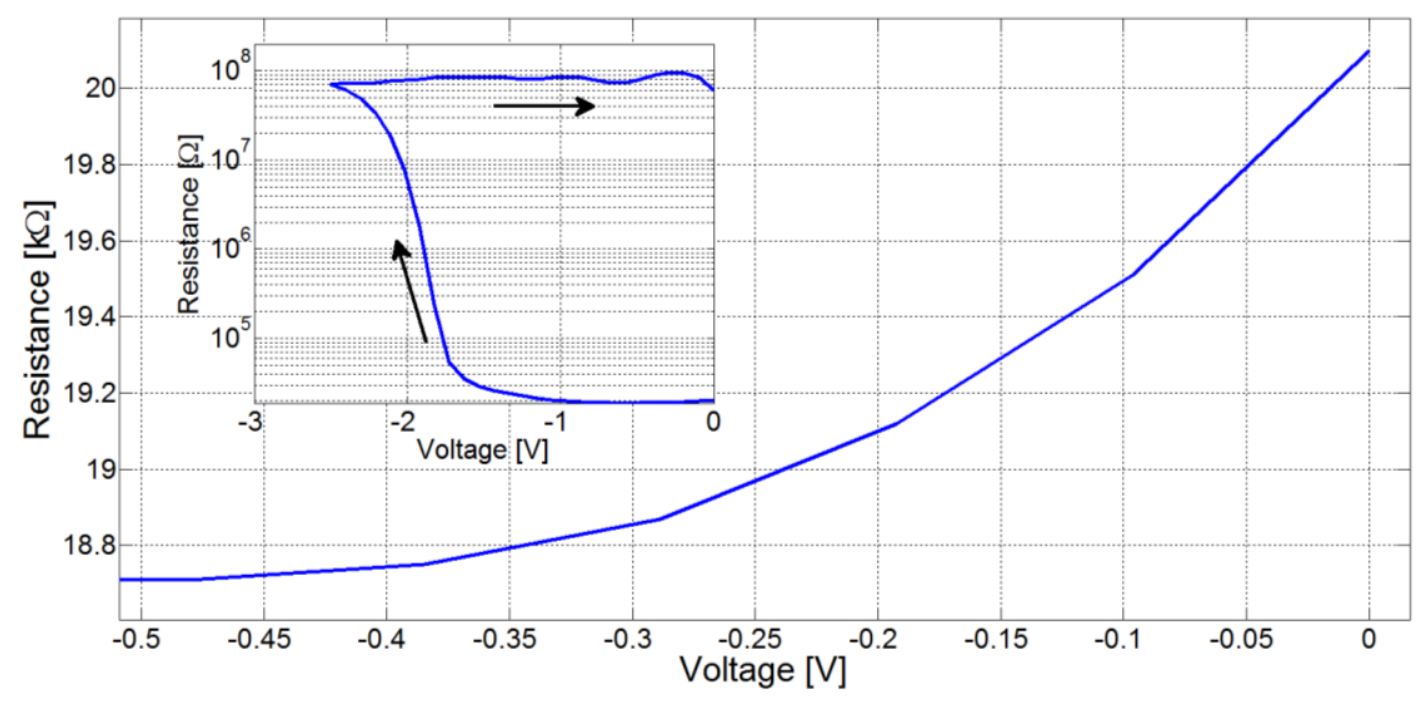

Figure 6.8 Resistance $v s$. applied voltage for a PET device during the oxidation of the polymer film

The overall change in resistance from $0 \mathrm{~V}$ to $-0.5 \mathrm{~V}$ was about $1.2 \mathrm{k} \Omega$, which compared with the change in resistance for the dedoping process $(\sim 45 \mathrm{M} \Omega)$ indicates that the polymer film is essentially completely doped with very few remaining redox sites 
available for further oxidation. This small additional oxidation is likely to involve the removal of protons from the PSS chain that enable PSS $^{-}$counter-ions to oxidize more EDOT monomers, as mentioned in section 3.4.1 and described by equation 3.1. The inset in Figure 6.8 shows the irreversible change in resistance caused by overoxidizing the polymer film with a maximum average resistance of $80 \mathrm{M} \Omega$. From this, an on/off ratio of 4290 was calculated for the irreversible change in resistance. A closer look at Figures 6.8 and 6.7 leads to the conclusion that this PET device could function as a rewritable memory component for positive voltages and as a write-once-read-many (WORM) device for negative voltages, which is a functionality that has been discussed in the literature [41].

The results given in Figures 6.8 and 6.7 can be combined and used with the integrated current to calculate a complete representation for the resistance as a function of intercalated charge as shown in Figure 6.9. This Figure indicates that the maximum intercalated charge was roughly $1.2 \mathrm{mC}$ and about 2.8 times larger than the expected value of $427 \mu \mathrm{C}$. However, the expected value was calculated by assuming that only a third of the available redox sites were able to participate in a redox reaction, as mentioned in section 6.3. 


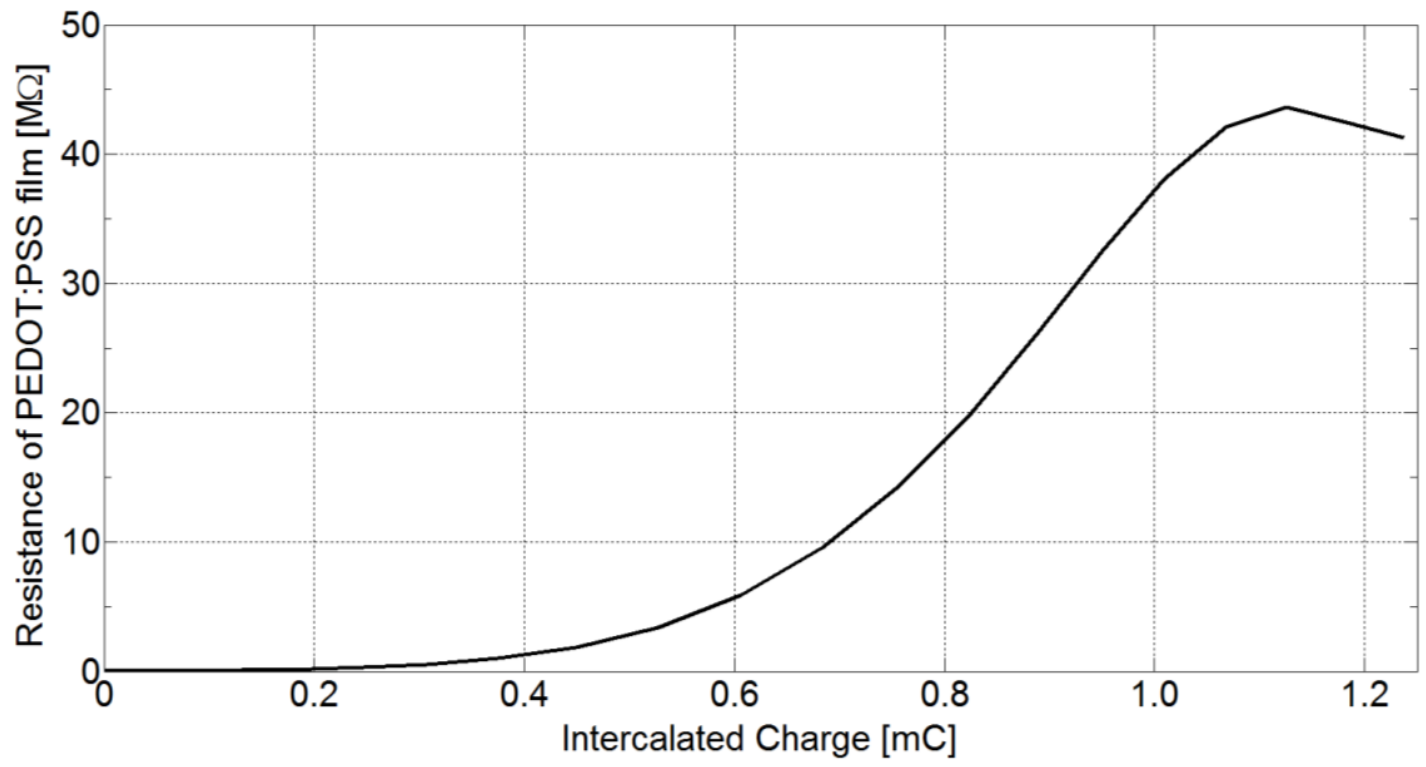

Figure 6.9 Resistance vs. Intercalated Charge for the polymer film in a PET device

This discrepancy might be attributed to possible side reactions that might have occurred inside the PET device or electrical leakage currents during the spectroelectrochemical experiment. For instance, in all the PET devices it was observed that an air bubble within the electrolyte-filled cavity appeared throughout the experiment. This was most likely caused by electrolysis of water impurities in the electrolyte (about 13ppm), which were comparable to the $\mathrm{Li}^{+}$ion concentration in the electrolyte.

The same information provided by Figure 6.9 can also be represented by the conductivity of the PEDOT:PSS active region as a function of concentration of intercalated $\mathrm{Li}^{+}$ions (shown in Figure 6.10). The inset in Figure 6.10 shows the previously mentioned relationship in a linear scale. This highlights the fact that this relationship had an approximate sigmoidal shape. 


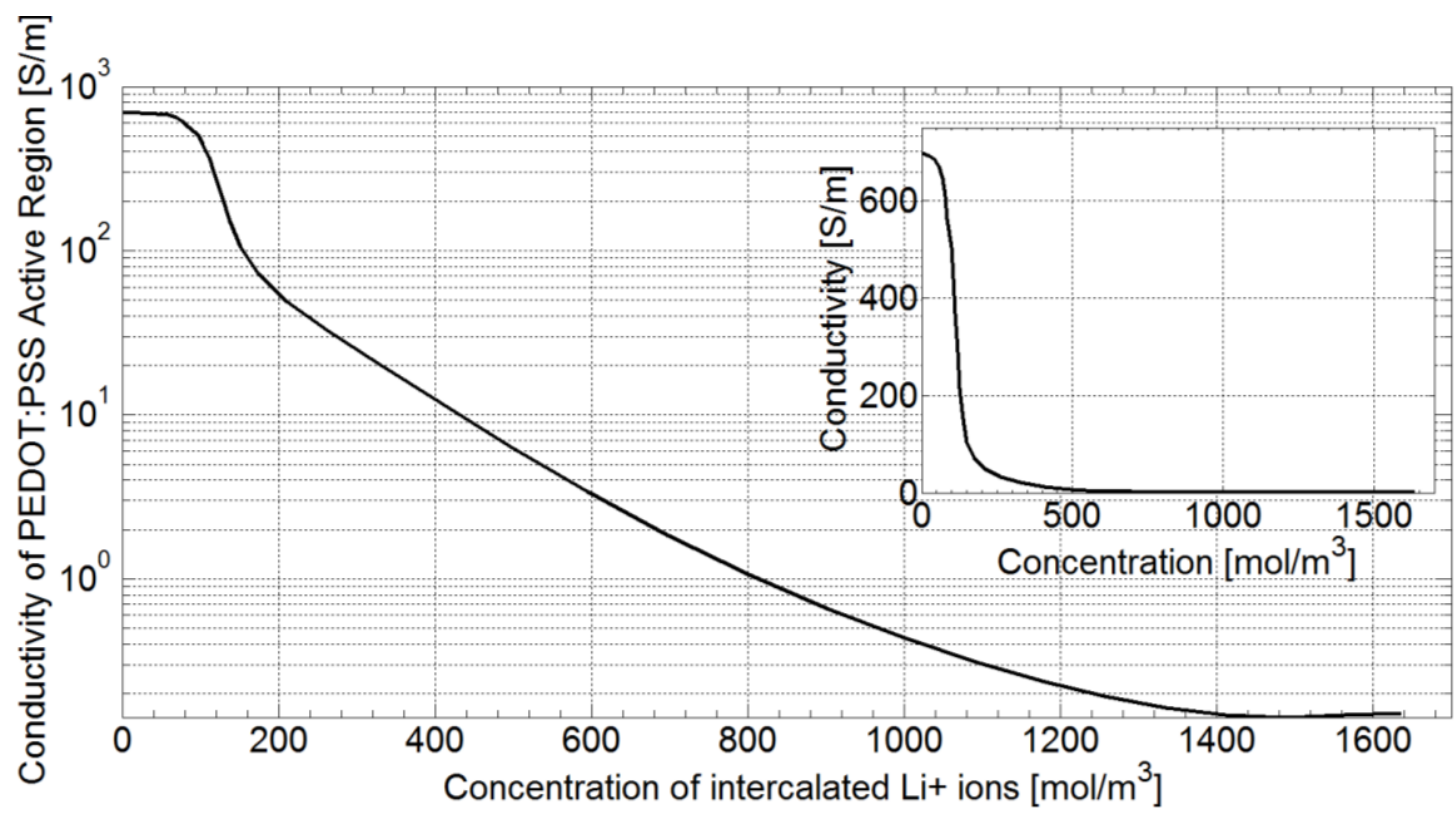

Figure 6.10 Conductivity $v s$. Intercalated $\mathrm{Li}^{+}$ion concentration for the polymer film in a PET device in $\log$ scale (Linear scale is shown in the inset)

The conductivity curve shown in Figure 6.10 was calculated by subtracting the unvarying resistance of the dry PEDOT:PSS regions $\left(2 \cdot R_{o}\right)$ from the compensated LCR measured resistance $\left(R_{P}\right)$, given by equation 6.2 , to obtain the active PEDOT:PSS resistance $\left(R_{A}\right)$. The total dry resistance $\left(2 \cdot R_{o}\right)$ was calculated to be $4.578 \mathrm{k} \Omega$. This value and the $R_{P}$ measurements were used to determine the conductivity using equation 6.4 , where the $L, W$ and $t$ are the length, width and thickness of the active PEDOT:PSS region. Figure 6.11 illustrates the relationship between $R_{o}, R_{P}$ and $R_{A}$.

$$
\begin{gathered}
\sigma[S / m]=\frac{L}{\left(R_{P}-R_{o}\right) W \cdot t} \\
\sigma[S / m]=\frac{7 m m}{\left(R_{P}-4.578 \mathrm{k} \Omega\right) 0.7 \mathrm{~mm} \cdot 1.6 \mu \mathrm{m}}
\end{gathered}
$$




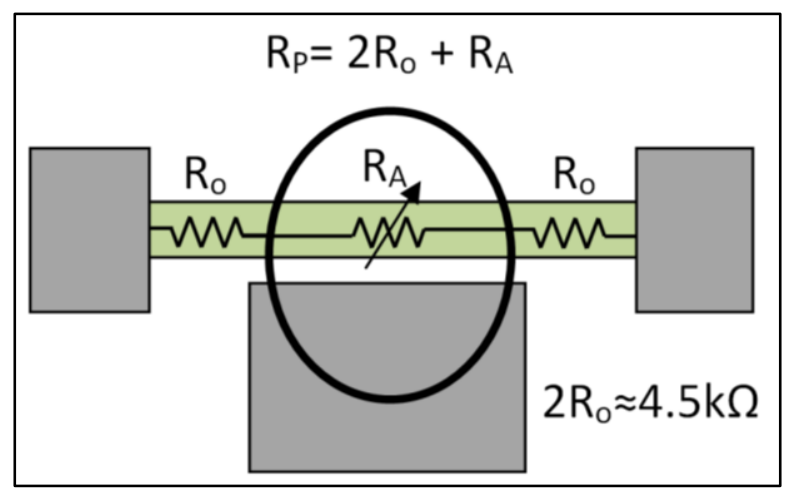

Figure 6.11 Illustration of total dry resistance $\left(2 R_{o}\right)$ and active resistance $\left(R_{A}\right)$ for the polymer film

The concentration of $\mathrm{Li}^{+}$ions $\left(N^{L i}\right)$ was determined by converting the intercalated charge into number of electrons and calculating the corresponding molar concentration for the volume of active PEDOT:PSS, which was $7.84 \times 10^{-6} \mathrm{~cm}^{3}$. Equation 6.5 summarizes the above operations required to obtain $N^{L i}$.

$$
\begin{gathered}
N^{L i}=\frac{\text { Charge }}{1.602 \times 10^{-19}[\mathrm{C}] \cdot 7.84 \times 10^{-12}\left[\mathrm{~m}^{3}\right] \cdot 6.0221 \times 10^{23}\left[\mathrm{~mol}^{-1}\right]} \\
N^{L i}\left[\frac{\mathrm{mol}}{\mathrm{m}^{3}}\right]=\frac{\text { Charge }}{7.56 \times 10^{-7}\left[\frac{\mathrm{C} \cdot \mathrm{m}^{3}}{\mathrm{~mol}}\right]}
\end{gathered}
$$

\subsubsection{Spectral Attenuation Measurements}

The resistance change observed in Figure 6.9 was also accompanied by a transformation in the spectral attenuation of the polymer film. Based on the optical properties of PEDOT:PSS given in section 3.6, it was expected that the following peaks would appear in the spectral attenuation: two peaks at low energies (NIR) for the doped state of the polymer and a peak due to interband electron transitions at a high energy (high VIS) for the undoped state. The two peaks associated with a doped state and related 
to the bipolaron states are referred to as bipolaron peaks [38]. Figure 6.12 shows the spectral attenuation of the polymer film in the PET device measured in situ during the spectroelectrochemical experiment where the peaks are indicated by the thick black arrows and the evolution of the spectrum is shown by the narrow black arrow. Each spectrum curve was measured at the end of the associated voltage step.

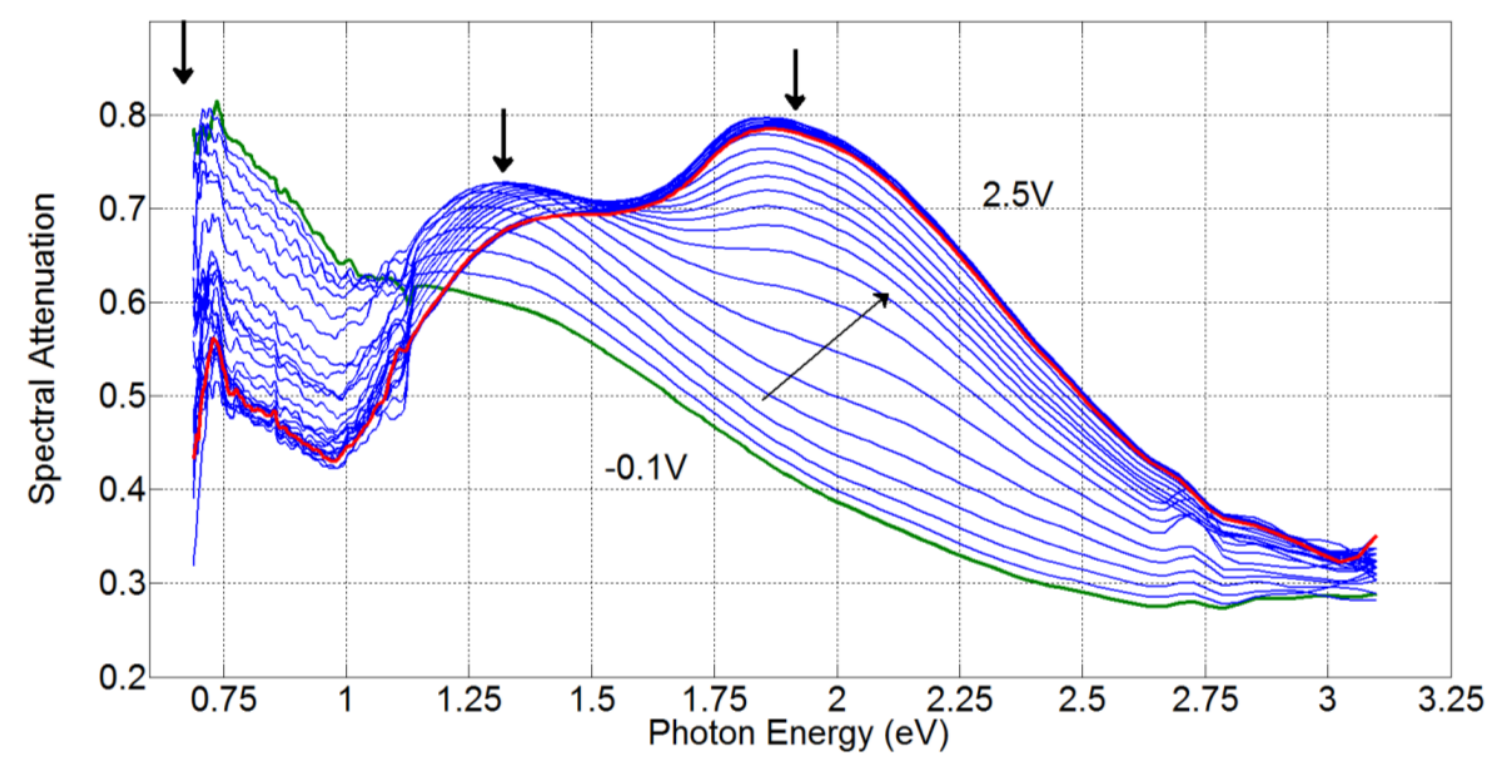

Figure 6.12 Spectral Attenuation of the PEDOT:PSS film in a PET device during dedoping. The spectral attenuation (SA) represents the ratio of the signal blocked by the assembled polymer sample and the total signal measured without the polymer sample (Equation 6.2).

The optical spectrum evolution shown in Figure 6.12 matches the previously mentioned expectations and was measured during the experiment in which the voltage waveform had a minimum and maximum of -0.1 to $2.5 \mathrm{~V}$. The undoped state (red) obtained at $2.5 \mathrm{~V}$ shows a strong peak centered at about $1.9 \mathrm{eV}$, which represents the interband $\left(\pi-\pi^{*}\right)$ transition. The doped state (green) was measured without an applied voltage and shows clear evidence of a strong single peak at $<0.7 \mathrm{eV}$, while a second peak is slightly visible between $1 \mathrm{eV}$ and $1.5 \mathrm{eV}$. The second peak emerged more clearly as the 
polymer was dedoped and was centered at about $1.3 \mathrm{eV}$. Similar results have been obtained for PEDOT:PSS thin films and other polythiophene polymers [38] [39]. The spectrum for the decreasing voltage portion of the experiment was identical to Figure 6.12.

\subsection{Conclusions}

The relationship between polymer conductivity and intercalated $\mathrm{Li}^{+}$ion concentration had a sigmoidal shape where the conductivity changed from $\sim 700 \mathrm{~S} / \mathrm{m}$ to $0.39 \mathrm{~S} / \mathrm{m}$. The minimum conductivity was obtained for a total of $\sim 1.2 \mathrm{mC}$ of intercalated charge. An on/off ratio of 2500 was calculated for the dedoping of the polymer by taking into account that the maximum conductivity of the doped polymer was $975 \mathrm{~S} / \mathrm{m}$. A hysteresis-like behaviour was observed during the reversible change in conductivity of the polymer. Overall, the relationship between the polymer main properties (conductivity and spectral attenuation) and intercalated $\mathrm{Li}^{+}$concentration was presented, which will be very useful in a future simulation of these polymer devices. 


\section{Chapter 7}

\section{Video Analysis of Lithium Intercalation into PEDOT:PSS Films}

\subsection{Introduction}

In order to obtain a qualitative picture of how $\mathrm{Li}^{+}$ions from an electrolyte intercalate into a PEDOT:PSS film, an in situ video capturing experiment was performed during the dedoping of the polymer film. In the case of a dedoping process, $\mathrm{Li}^{+}$ions intercalate from the electrolyte into the polymer and a blue coloration is observed due to an increasing ion density that will be referred to as a "rising front". A blue decolouration due to re-doping of the polymer will be referred to as "falling font". The set of experiments were performed on two types of PEDOT:PSS devices using different applied voltages. The videos were analyzed using a MatLab program that calculated the colour difference between each frame and a reference frame. This results in a matrix of colour difference amplitudes, which translate into a blue coloration or decolouration. The rising or falling front was estimated by determining the location at which the amplitude was $50 \%$ of the maximum amplitude for a specific frame. 


\subsection{Device Geometry}

The first geometry chosen for the PEDOT:PSS devices consisted of two graphite terminals connected by a PEDOT:PSS channel with a length and width of approximately $9.9 \mathrm{~mm}$ and $0.7 \mathrm{~mm}$, respectively, as shown in Figure 7.1. These devices were labeled as single channel devices.

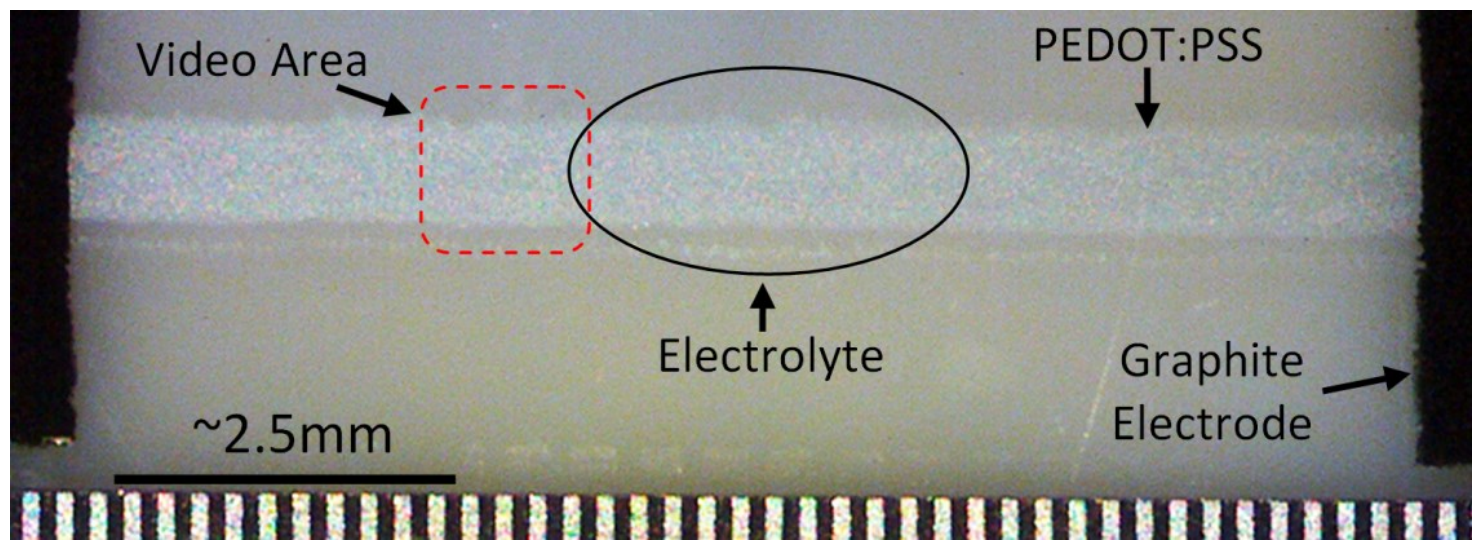

Figure 7.1 A single channel device. The black circle represents the area where the electrolyte was applied and the red dotted rectangle represents the region where the camera was focused.

The second type of PEDOT:PSS devices had the same geometry but the polymer channel was cut with an Exacto knife near one of the electrodes, which created a gap in the order of $0.1 \mathrm{~mm}$, as shown in Figure 7.2 (left \& right). This gap separated a short region $(S R)$ and a long region $(L R)$ of PEDOT:PSS. This second type of device was called a notch device. 


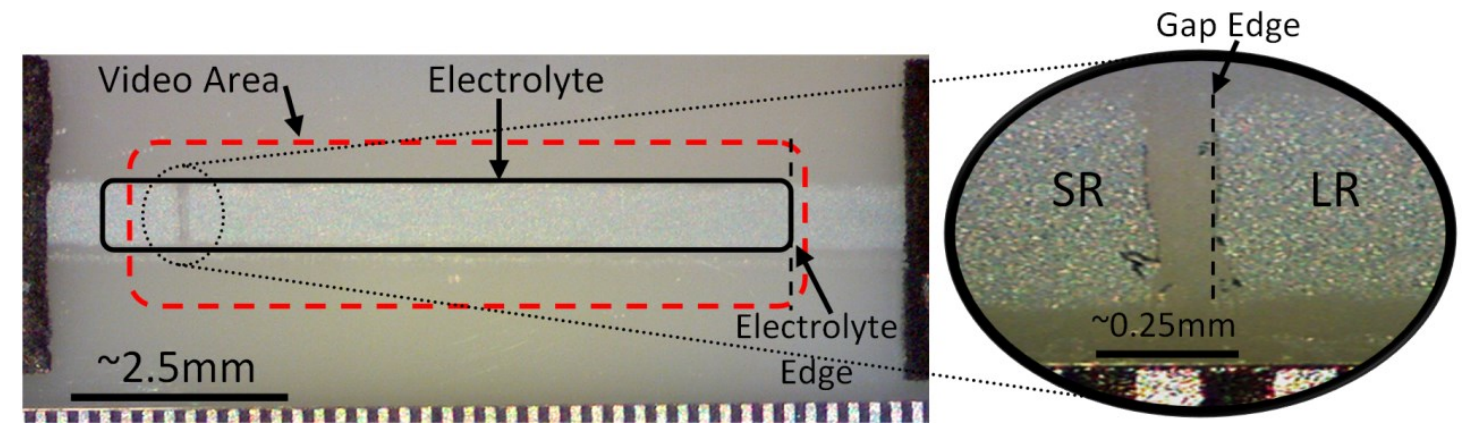

Figure 7.2 (left) A notch device and (right) a close up of the gap between the Short Region $(S R)$ and Long Region $(L R)$ of PEDOT:PSS.

\subsection{Experiment Setup and Explanation}

In order to dedope the polymer film a voltage was applied between the two graphite end contacts using a Keithley 595 SMU and a probing station. The SMU was used to monitor the current and ensure that the expected current decay was observed and that there were no abnormalities during the redox reaction. The probing station consisted of a horseshoe metal stand, two mechanical gold probes, and an optical microscope with a digital microscope camera placed into an eyepiece, as shown in Figure 7.3. The experiment was controlled by a computer using HP VEE and all the data was collected by GPIB and USB interfaces. 


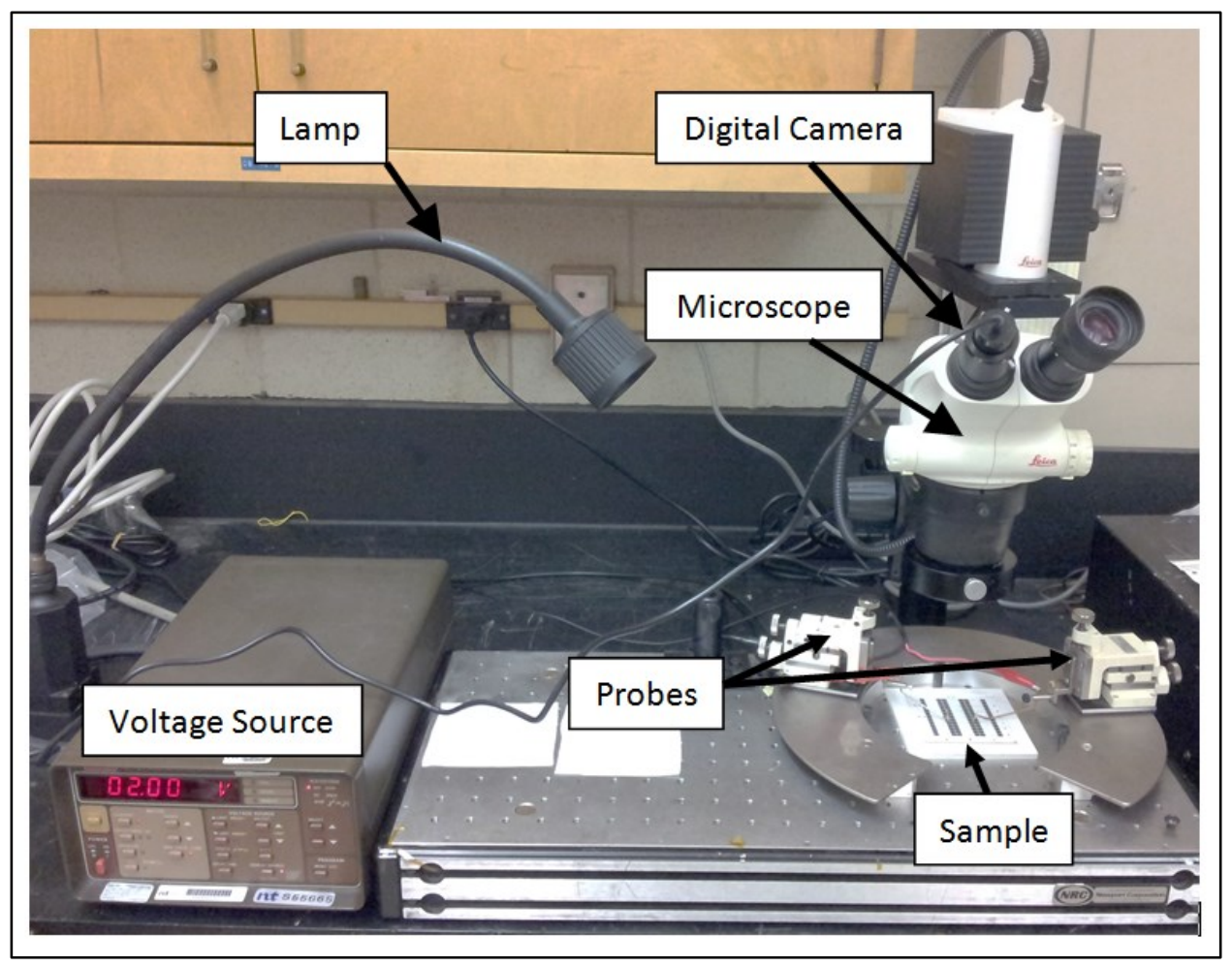

Figure 7.3 Setup for video analysis of $\mathrm{Li}^{+}$ion intercalation into PEDOT:PSS thin films

In the case of the single channel device, the gold probes were positioned on the graphite contacts and a few drops of a $1.0 \mathrm{M} \mathrm{LiClO}_{4}$ electrolyte were applied on top and in the middle of the polymer film using a syringe. The digital camera was focused on one side of the electrolyte drop so that part of the electrolyte and dry polymer were visible (Figure 7.1). A constant voltage was applied to the device for 10 minutes. A total of five videos were recorded for five different voltage amplitudes that ranged from $2 \mathrm{~V}$ to $5 \mathrm{~V}$ in steps of $1 \mathrm{~V}$ and also included $1.5 \mathrm{~V}$. During the 10 minute period, the camera recorded the blue coloration of the polymer due to the dedoping process. The purpose of this experiment was to capture the ion movement into dry polymer regions (i.e. regions of polymer without electrolyte applied on top). 
The notch device had the same set up as the single channel device, but the camera was focused onto the $L R$ polymer that was underneath the electrolyte, hence the dry polymer regions were of no interest in this case. Also, the electrolyte was applied such that it would cover a larger area of the $L R$ polymer than the $S R$ polymer (Figure 7.2). In this case, the voltage applied was switched from $-0.5 \mathrm{~V}$ to $1.7 \mathrm{~V}$ with constant voltage duration of 20 seconds and the ground terminal was placed on the electrode that was attached to the $L R$ polymer. Therefore, the $L R$ polymer was dedoped and re-doped during the applied voltage of $1.7 \mathrm{~V}$ and $-0.5 \mathrm{~V}$, respectively. This dedoping and re-doping was repeated five times. The purpose of this experiment was to observe how the ions would intercalate into wet polymer regions (i.e. regions of polymer with electrolyte applied on top).

\subsection{Video Results Analysis}

\subsubsection{Single Channel PEDOT:PSS Device}

Each 10 minute video file was transformed into a sequence of image files that had a spatial resolution of 500 pixels $/ \mathrm{mm}$ and were analyzed using an image processing program written in MatLab. This program detected a rising front by calculating the maximum change in blue coloration in the polymer during dedoping and determined the ion front displacement into the dry polymer region and speed. In each 10 minute section, the first minute was analyzed at a frame rate of 5 frames/seconds (fps) and then the analysis for the entire 10 minutes was done at $1 \mathrm{fps}$. Both of these analyses determined the speed and displacement of the ion front by averaging over 10 trajectories along the center of the polymer film and parallel to the ion front motion. 
The analysis for the dedoping process showed that the rising front motion was quicker for higher applied voltage as expected, but only a maximum displacement of approximately $0.2 \mathrm{~mm}$ could be reached by the rising front regardless of the applied voltage (Figure 7.4).

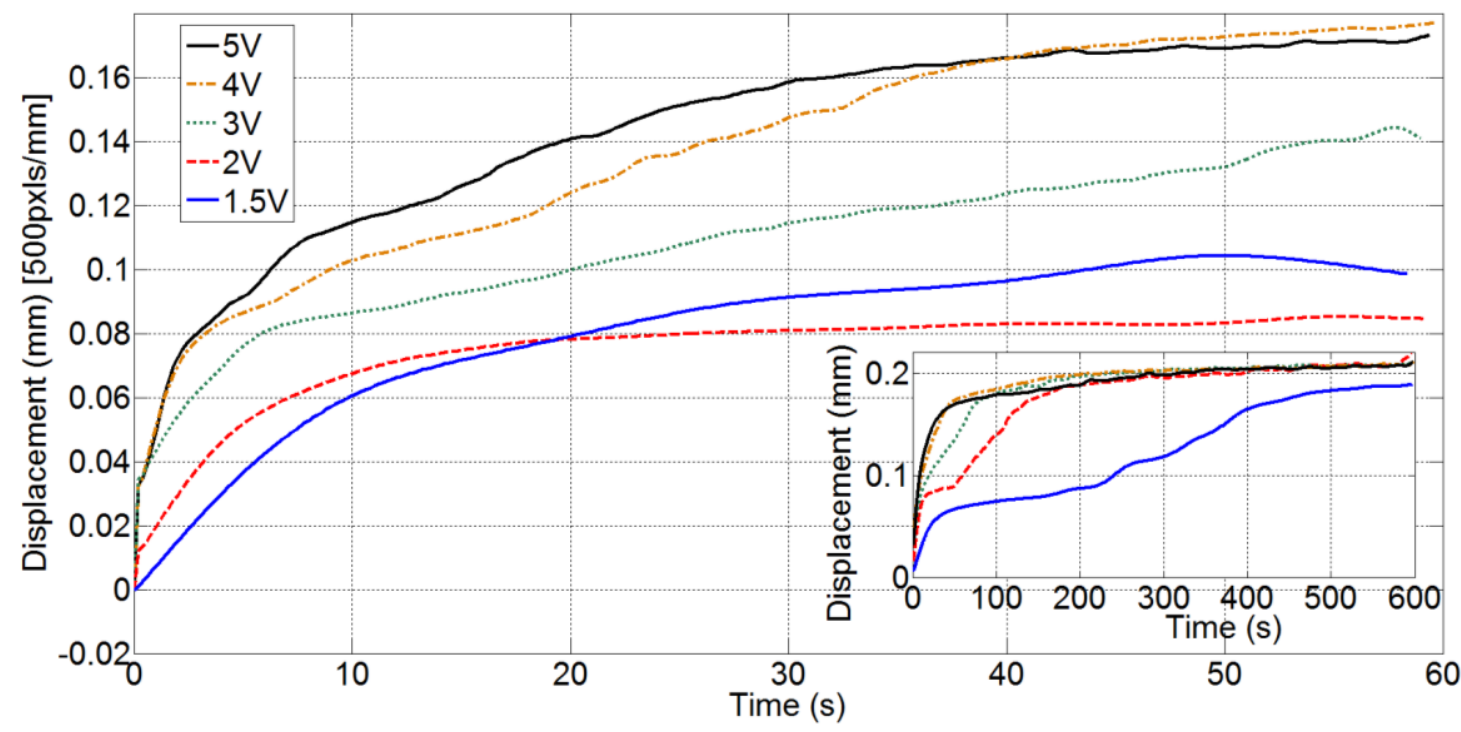

Figure 7.4 Displacement over time of the rising front for various applied voltages during the $1^{\text {st }}$ minute and (inset) during the entire 10 minutes.

Figure 7.4 shows that during the first minute of dedoping, the displacement $(\delta)$ of the rising front over time $(t)$ can be approximated by a power function in the form of equation 7.1, where $\beta$ and $\alpha$ can be experimentally determined.

$$
\delta=\beta t^{\alpha}
$$

Moreover, the inset in Figure 7.4 reveals that this power law approximation is no longer valid after roughly the first minute. The maximum displacement and gradual decrease in speed of the rising front can be associated with a decrease in mobility from the edge of the electrolyte towards the dry PEDOT:PSS regions. Figure 7.5 shows the 
logarithmic relationship between displacement and time which was used to solve for $\alpha$ and $\beta$, shown in equation 7.1, at each voltage. One can see from Figure 7.5 that the power law approximation holds fairly well for the displacement of ion at $3 \mathrm{~V}, 4 \mathrm{~V}$ and $5 \mathrm{~V}$ during the first minute. However, at low voltages $(1.5 \mathrm{~V}$ and $2 \mathrm{~V})$ the displacement of ions began to deviate from the power law approximation.

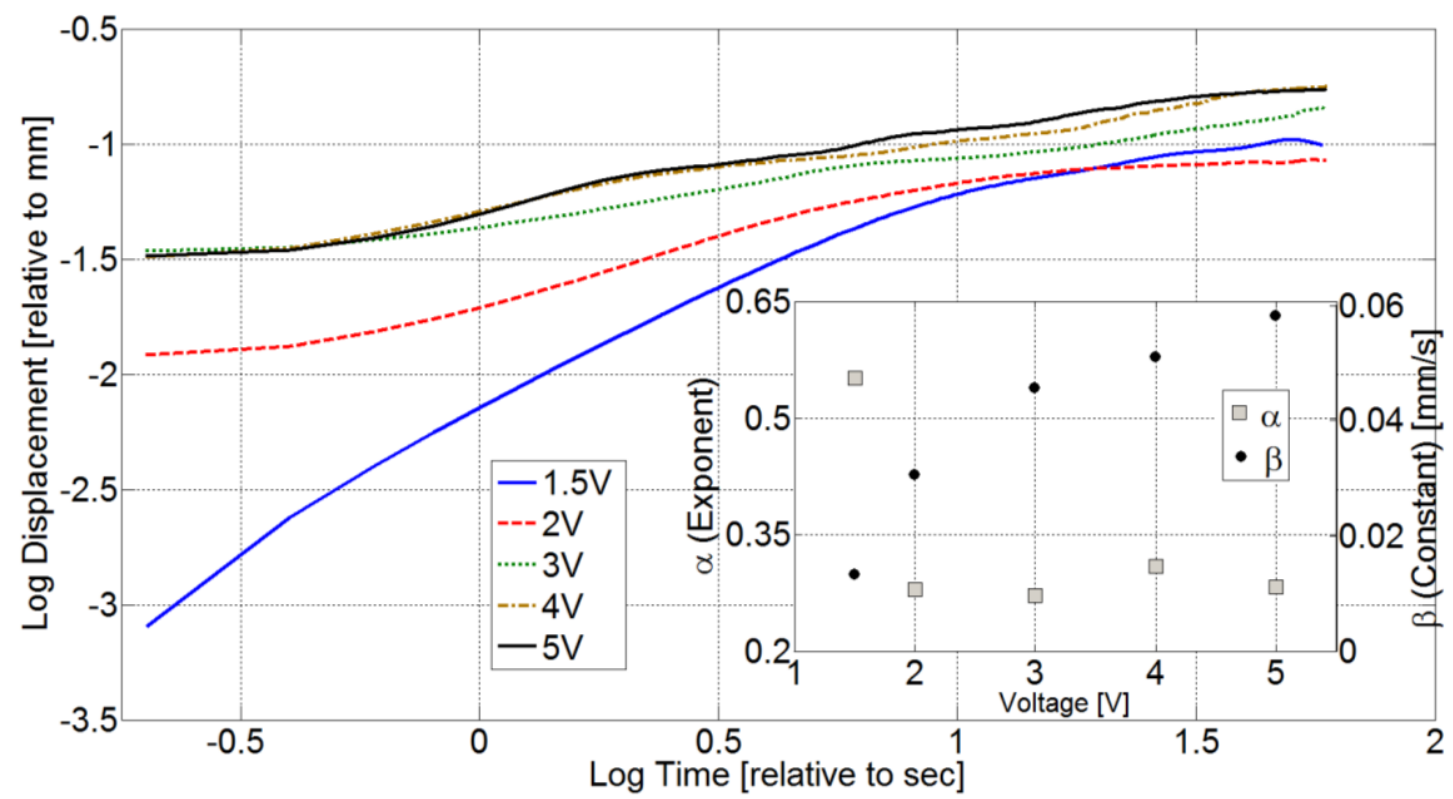

Figure 7.5 Logarithmic relationship between displacement and time during the first minute of dedoping. (Inset) $\alpha$ and $\beta$ values extracted by fitting experimental data to the power law

Figure 7.5 shows that $\alpha$ was fairly constant with an average of 0.286 for all the voltages except for $1.5 \mathrm{~V}$ in which $\alpha$ increased to 0.552 . On the other hand, the $\beta$ constant gradually decreases as the voltage decreases, which is due to the fact that ions migrate faster at higher applied voltage. Overall, as the voltage increases, $\alpha$ and $\beta$ approach values of 0.286 and $0.06 \mathrm{~mm} / \mathrm{s}$, respectively. The derivative of equation 7.1 gives the rising front speed $(v)$ which is proportional to $t^{-\gamma}$ where $\gamma$ is given by $1-\alpha$ as shown in equation 7.2 


$$
v=\beta \alpha t^{-(1-\alpha)}=\beta \alpha t^{-\gamma}
$$

Based on the values for $\alpha$, the calculated $\gamma$ value for $1.5 \mathrm{~V}$ is 0.448 and the average $\gamma$ value for $2 \mathrm{~V}$ to $5 \mathrm{~V}$ is 0.715 . A similar relationship between front speed and time is mentioned in the literature for another polythiophene conjugated polymer called of poly(3-hexylthiophene) (P3HT). In that case, $\gamma$ was determined to be between 0.35 and 0.45 for voltages between $1.2 \mathrm{~V}$ and $950 \mathrm{mV}$ [42]. These values are close to the calculated $\gamma$ value for $1.5 \mathrm{~V}$ in this work.

Figure 7.6 shows a spatial illustration of the rising front motion for 7 different times and an applied voltage of $5 \mathrm{~V}$, where a dark blue colour represents a high ion density and the rising front moves from right to left.

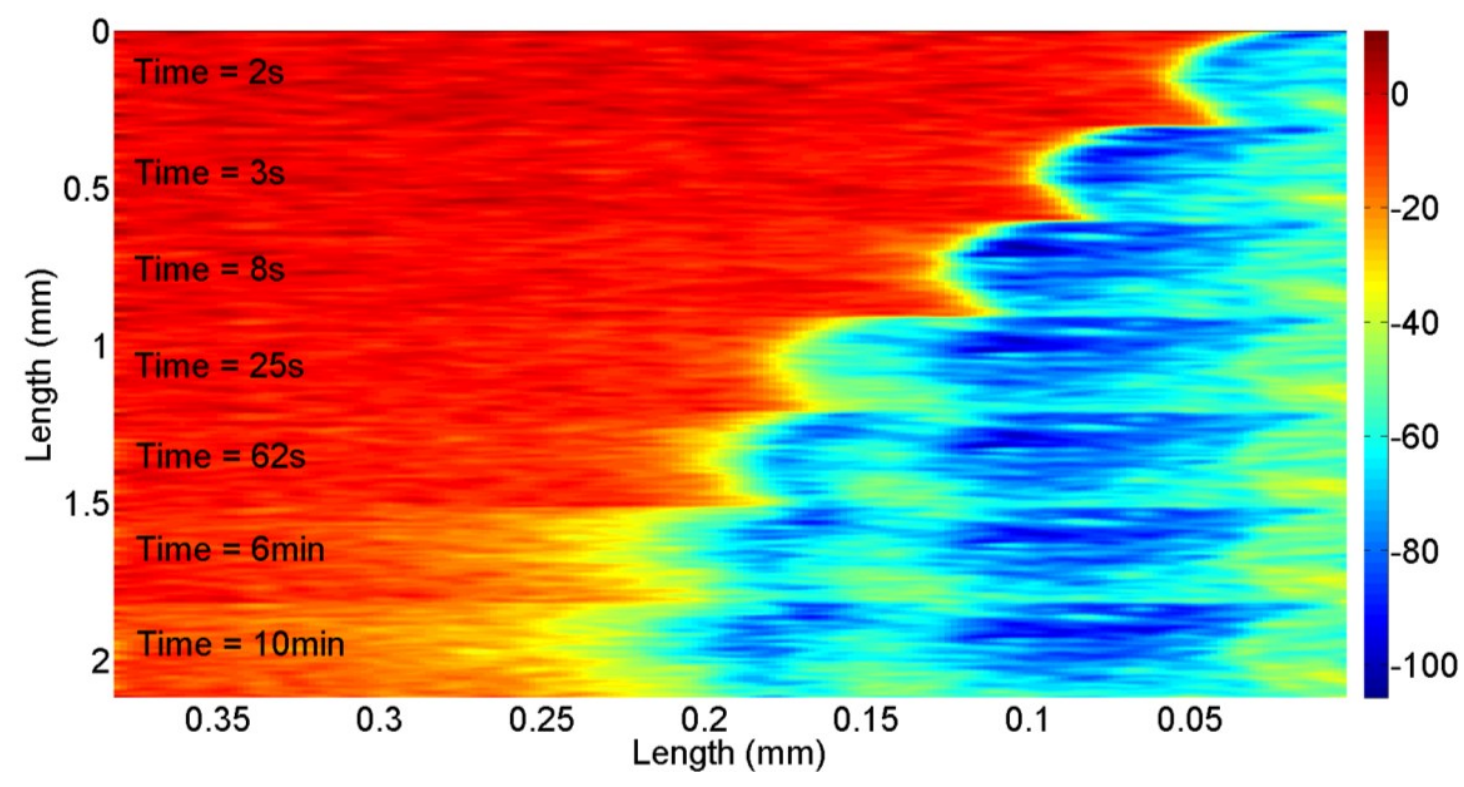

Figure 7.6 Spatial and Temporal evolution of the rising front for an applied voltage of $5 \mathrm{~V}$ during 10 minutes. 


\subsubsection{Notch PEDOT:PSS Device}

The video captured for the notch device was analyzed by dissecting the video into sections that were 20 seconds long and contained footage of the $L R$ polymer being dedoped or re-doped. It is important to remember that the $L R$ polymer studied is covered by an electrolyte. The image sequence created for each 20 second video was done at 25

fps and had a spatial resolution of 86 pixels $/ \mathrm{mm}$. The analysis was done by averaging over 5 trajectories along the center of the polymer and parallel to the ion front motion. There was a total of 5 separate dedoping videos, 4 re-doping videos and a single video where the polymer was redoped without any applied voltage and was allowed to reach equilibrium over 90 seconds.

\subsubsection{Dedoping Videos}

The analysis for the 5 dedoping videos confirmed a common rising front behaviour among the 5 videos, which consisted of an ion intercalation that began at the gap edge and gradually moved towards the electrolyte edge (refer to Figure 7.2 for the edges locations). The following Figures illustrate the results for the first dedoping video which is representative of the results for the remaining four videos. In this section, a high ion density was represented by a dark blue colour. Figure 7.7 shows the motion of the rising front throughout the polymer at 8 different times. 


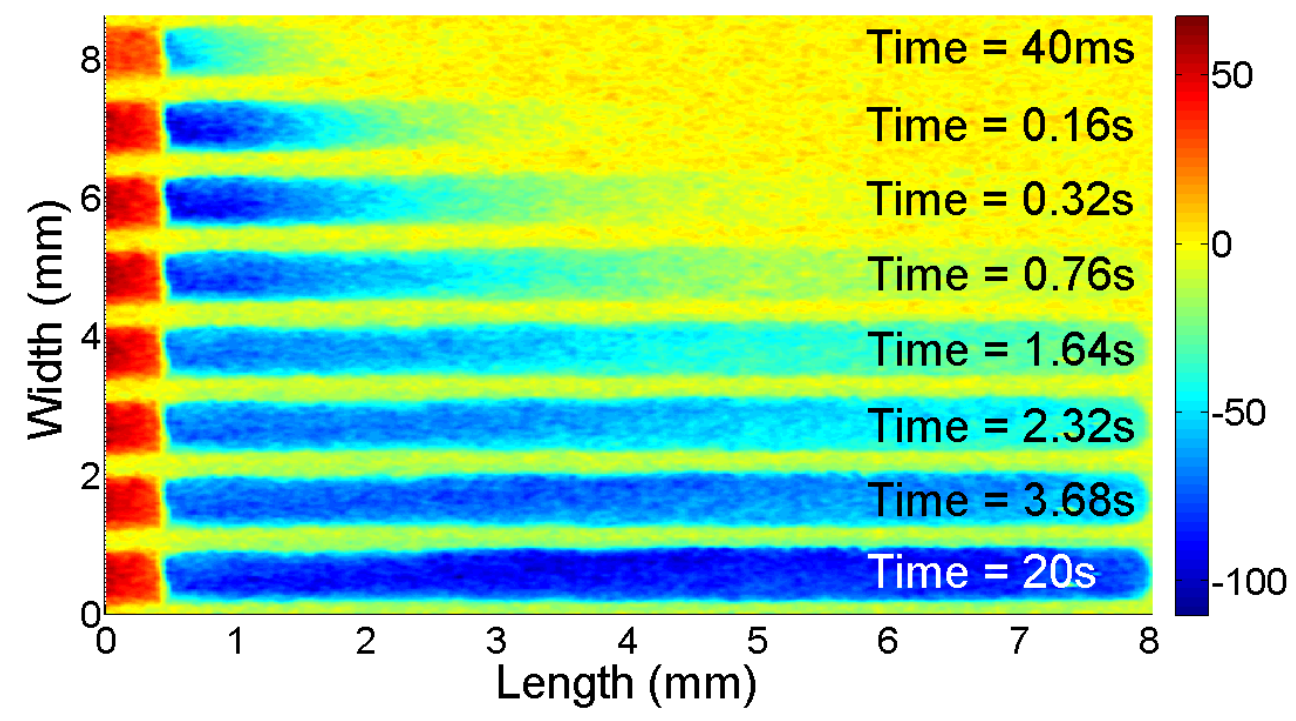

Figure 7.7 Spatial and Temporal evolution of the rising front for an applied voltage of $1.7 \mathrm{~V}$ during 20 s.

Figure 7.8 shows the relative ion density increase along a cross section parallel to the polymer length and through its center at the same 8 points in time shown in Figure 7.7.

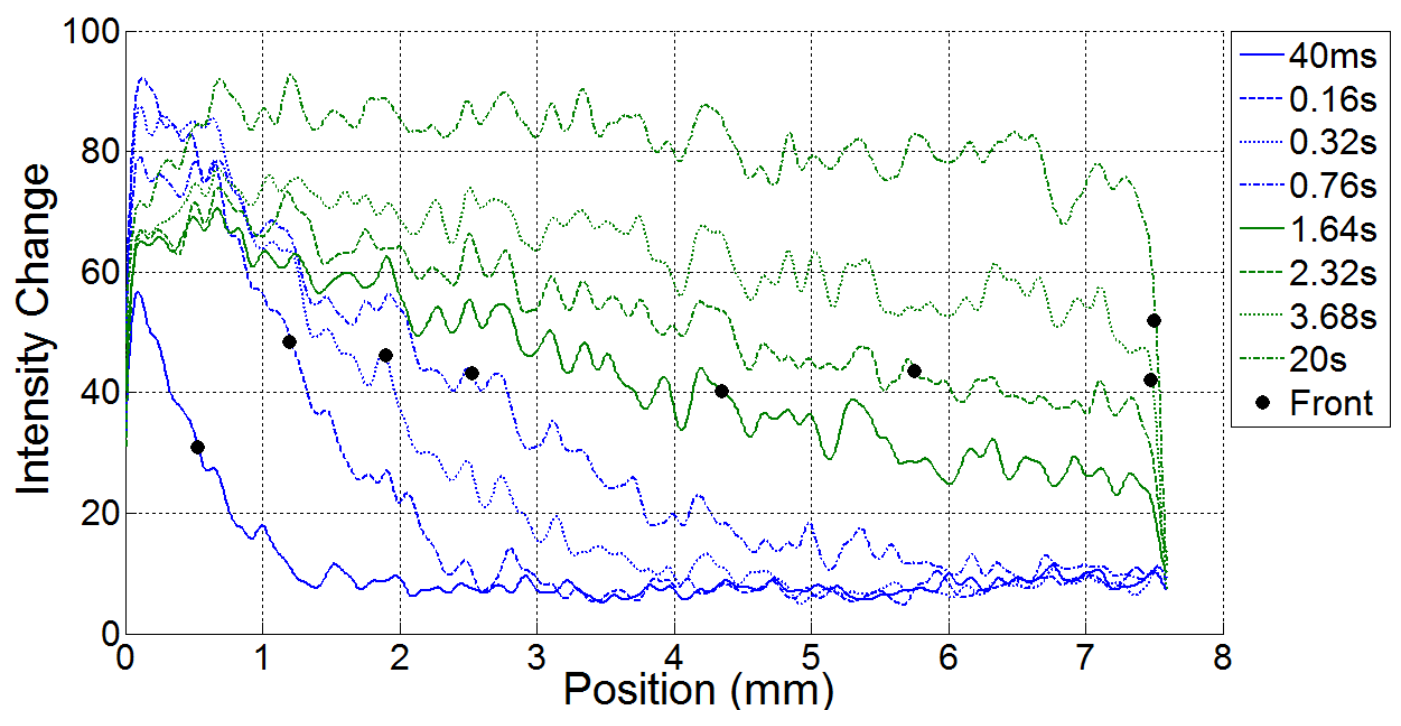

Figure 7.8 Spatial and temporal evolution of the rising front through a cross sectional path along the center of the polymer and for an applied voltage of $1.7 \mathrm{~V}$ during $20 \mathrm{~s}$. 
Both Figures 7.7 and 7.8 clearly show that a rising ion front developed and maintained a distinguishable front during the first 0.7 seconds approximately. Afterwards, the front diffused until it reached the electrolyte edge and began to accumulate uniformly throughout the polymer. In Figure 7.8, the black dot represents the point in a given curve where the MatLab algorithm assigned the front position $(50 \%$ of maximum intensity change), which becomes less meaningful after 0.76 seconds due to the diffusion previously mentioned.

Furthermore, the time dependence of the rising front displacement as calculated by the MatLab algorithm is plotted in Figure 7.9 for all the 5 dedoping videos. The average ion speed was calculated during the first 2 seconds of rising front motion for each dedoping video as shown the inset of Figure 7.9. One can see in Figure 7.9 that the rising front reached the electrolyte edge, located at about $7.5 \mathrm{~mm}$ from the gap edge, within a time interval that ranged from 3.5 to 4.5 seconds. Figure 7.9 shows that, in contrast to Figure 7.4, the rising front moved with an approximately constant speed until it reached the electrolyte edge. The average ion speed was determined to be $2.81 \mathrm{~mm} / \mathrm{s}$. 


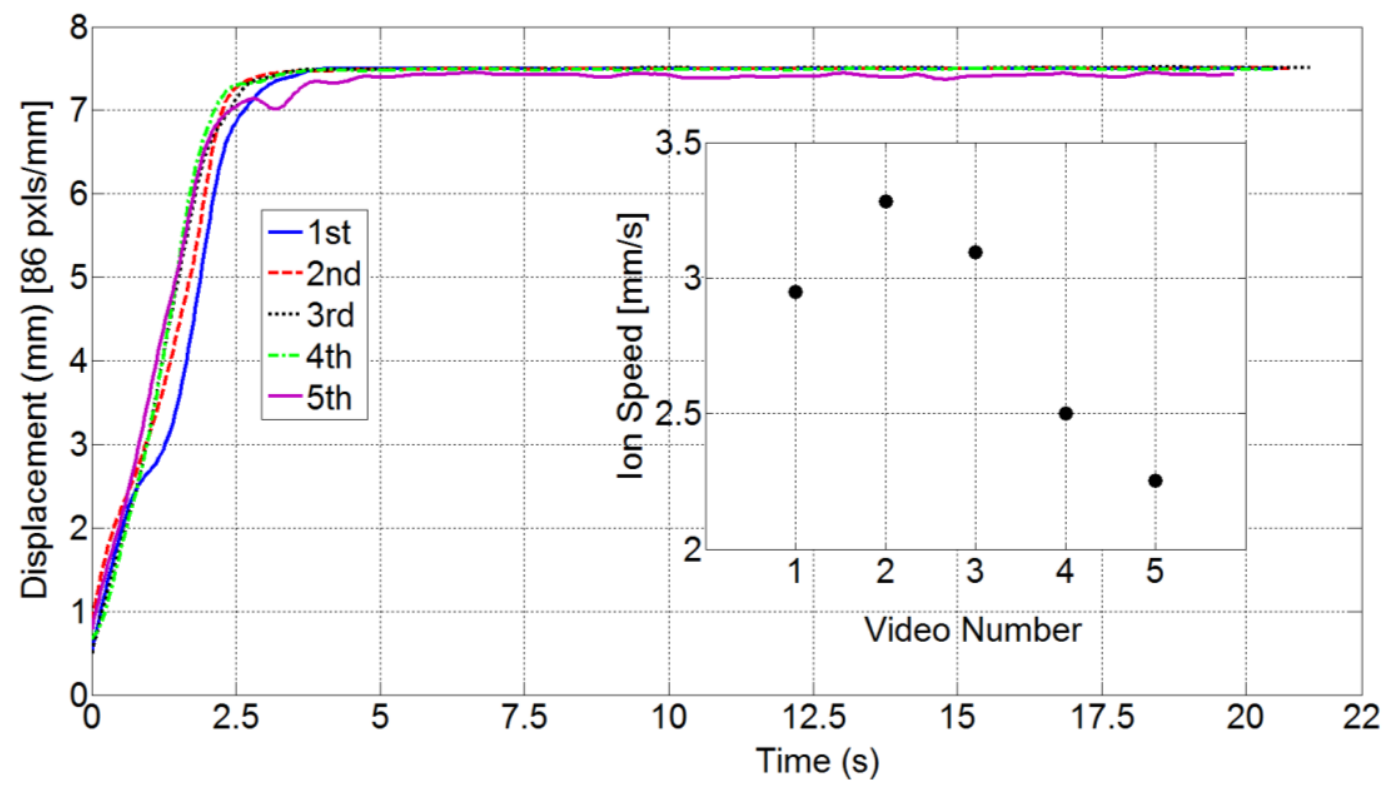

Figure 7.9 Displacement of rising front over time along the center of the PEDOT:PSS film during dedoping at $1.7 \mathrm{~V}$ for all 5 videos. (Inset) Average ion speed during the initial $2 \mathrm{~s}$ of rising front motion for each video

\subsubsection{Re-doping Videos}

The analysis performed on the four re-doping videos showed a striking difference in the falling front evolution as compared to the rising front evolution. The results shown in the subsequent Figures were obtained for the first re-doping video and are representative of the results obtained for the remaining three videos. In the case of these re-doping videos, the falling front represents a decrease in ion density and will be denoted by a red colour to differentiate it from the rising front in the previous section. During the redoping of the polymer, the $\mathrm{Li}^{+}$ions migrate from the polymer into the electrolyte. This ion migration occurs first at the gap and electrolyte edges and then it progresses toward the middle of the polymer until the ions are entirely absent throughout the polymer as shown in Figure 7.10. 


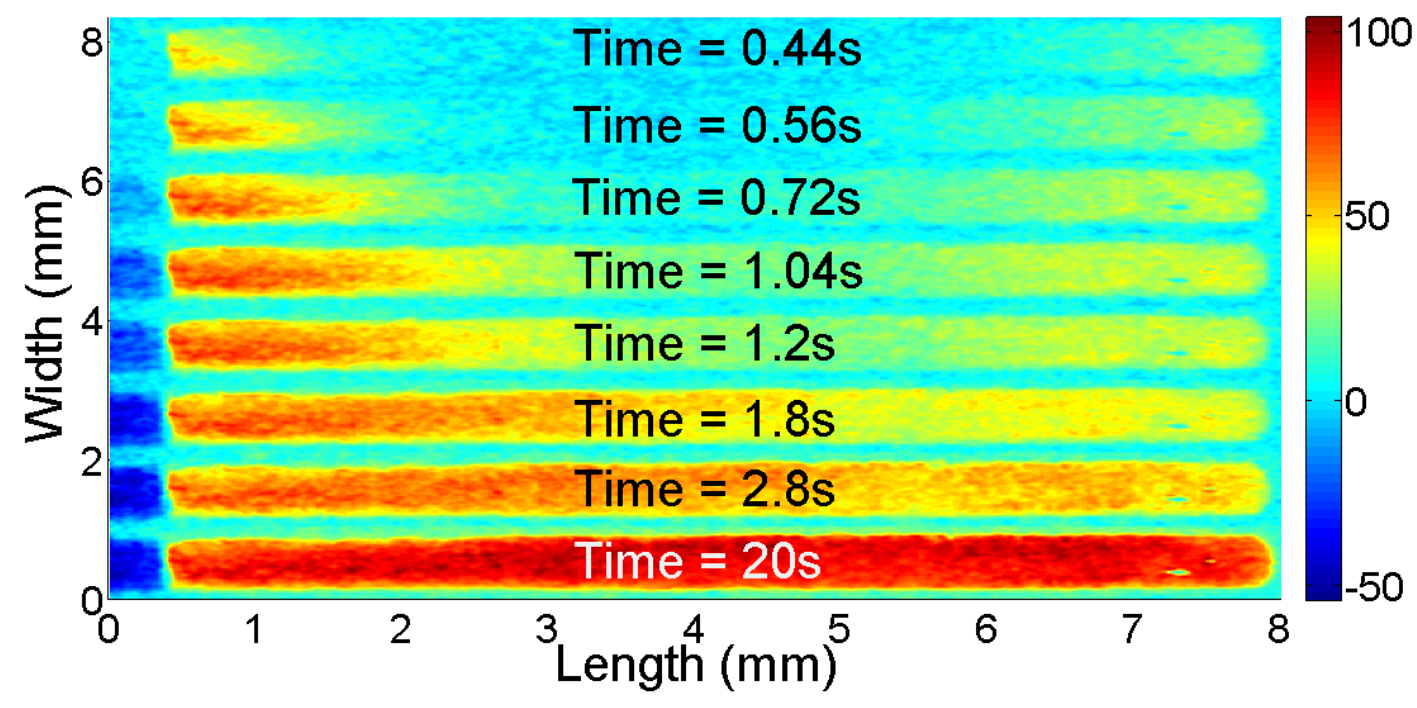

Figure 7.10 Spatial and Temporal evolution of the falling front for an applied voltage of $-0.5 \mathrm{~V}$ during $20 \mathrm{~s}$

Figure 7.10 shows that two falling fronts developed at both ends of the $L R$ polymer and moved towards the middle of the polymer. This ion migration behaviour is also illustrated in Figure 7.11, which shows the relative ion density decrease along a cross section parallel to the polymer length and through its center at the same 8 points in time shown in Figure 7.10. Due to the ion dynamics, the MatLab algorithm could not detect a front, and therefore no black dots were included in Figure 7.11. 


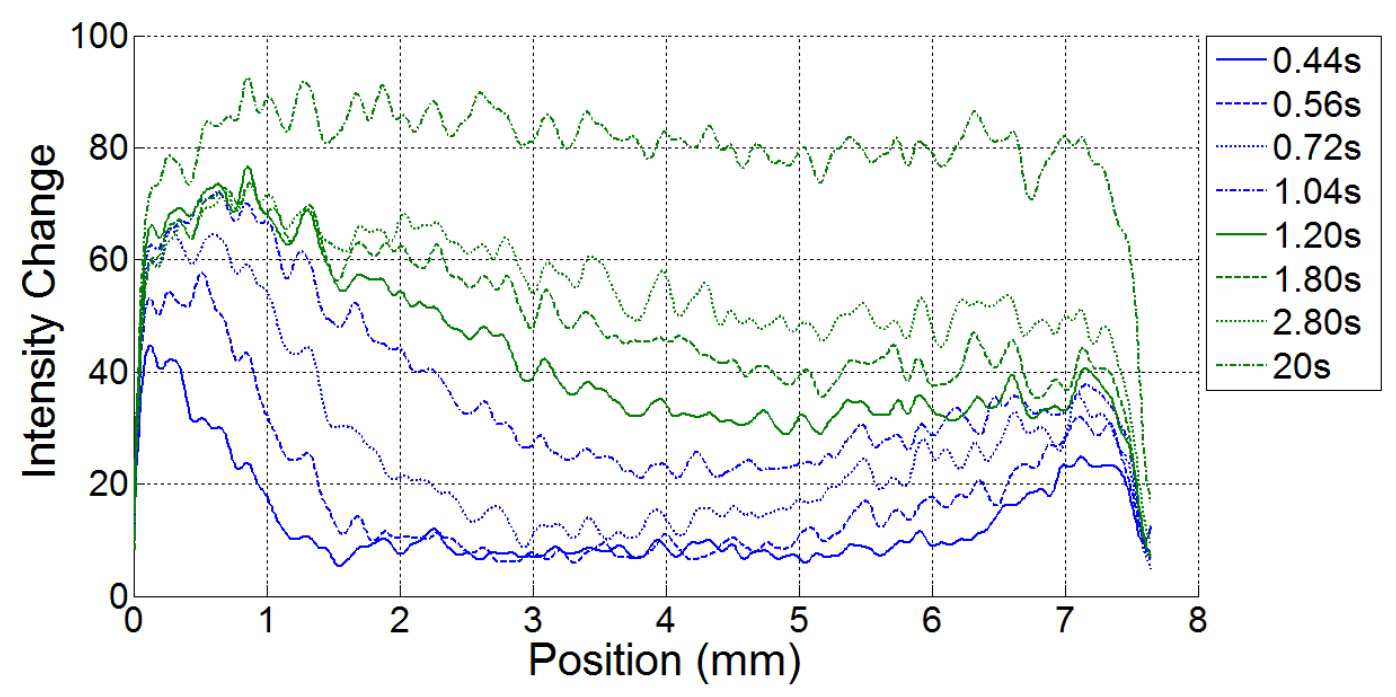

Figure 7.11 Spatial and temporal evolution of the falling front through a cross sectional path along the center of the polymer and for an applied voltage of $-0.5 \mathrm{~V}$ during $20 \mathrm{~s}$.

The final re-doping cycle was done by removing the voltage source and allowing the $\mathrm{Li}^{+}$ions to diffuse out of the polymer without any applied electric field. The analysis for this cycle shows that the ions de-intercalated uniformly from the polymer without any sign of an ion front, but it took more time for the ions to diffuse out of the polymer. Also, there was a remnant ion density in the polymer even after 90 seconds (Figure 7.12). In order to provide a clear illustration of this ion diffusion, a high ion density is denoted by a dark blue colour and the de-intercalation of these ions is represented by fading of the blue colour as shown in Figure 7.12. 


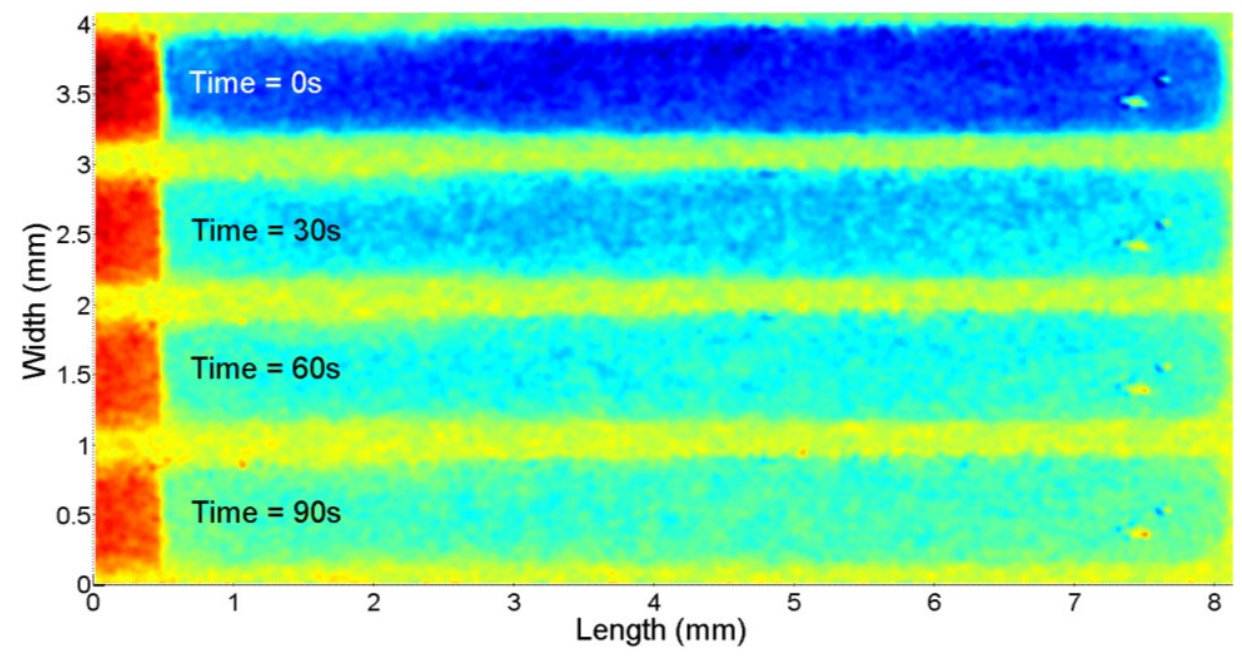

Figure 7.12 Spatial and Temporal evolution of the relative ion density for the re-doping of the $L R$ polymer without an applied voltage and during $90 \mathrm{~s}$

\subsection{Conclusions}

Although the shown measurements of ion intercalation distance and ion speed were limited in accuracy by the low spatial and optical resolution of the video capturing equipment, a clear qualitative behaviour of the ion intercalation was shown for a notch and single channel PEDOT:PSS devices. A power-law speed-time relationship was obtained for the ion migration from electrolyte to dry polymer regions for the single channel device. In the case of the Notch structure, the ion front speed from the gap edge to the electrolyte edge was calculated to be $2.81 \mathrm{~mm} / \mathrm{s}$. 


\section{Chapter 8}

\section{Diode-Like Behaviour of Asymmetric PEDOT:PSS}

\section{Devices}

\subsection{Introduction}

A set of PEDOT:PSS devices were fabricated with a varying asymmetry in the width of the polymer film. The AC electrical response of these devices was measured and the current-voltage (I-V) curves for each device were extracted. The frequency, voltage amplitude and geometry dependencies on the electrical response of the devices were illustrated and explained.

\subsection{Device Geometry}

Each asymmetric PEDOT:PSS device (APD) consisted of two silver contacts connected by a polymer channel, which was made up of two regions of equal length but with narrow and broad widths, respectively. A total of 5 devices were used in which the ratio between the narrow-width and broad-width regions was varied from 1 (symmetric) to about 13 . Tables 8.1 and 8.2 show all relevant dimensions, width ratios and the initial resistance for each device. Figure 8.1 shows the 5 devices used in this experiment. Every device had different widths and device APD-1 is in fact a symmetric device. 
Table 8.1 Dimensions and initial resistance for each asymmetric PEDOT:PSS Device

\begin{tabular}{|c|c|c|c|c|c|c|c|}
\hline & \multicolumn{3}{|c|}{ Region 1 - Broad Width } & \multicolumn{2}{c|}{ Region 2- Narrow Width } & \\
\hline Device & $\begin{array}{c}\text { Length } \\
(\mathbf{m m})\end{array}$ & $\begin{array}{c}\text { Width } \\
(\mathbf{m m})\end{array}$ & $\begin{array}{c}\text { Area } \\
\left(\mathbf{m m}^{\mathbf{2}}\right)\end{array}$ & $\begin{array}{c}\text { Length } \\
(\mathbf{m m})\end{array}$ & $\begin{array}{c}\text { Width } \\
(\mathbf{m m})\end{array}$ & $\begin{array}{c}\text { Area } \\
\left(\mathbf{m m}^{\mathbf{2}}\right)\end{array}$ & $\begin{array}{c}\text { Resistance } \\
(\mathbf{k} \mathbf{)})\end{array}$ \\
\hline APD-1 & 4.21 & 10.62 & 44.68 & 4.21 & 10.62 & 44.66 & 0.82 \\
\hline APD-2 & 4.16 & 10.56 & 43.93 & 3.73 & 5.06 & 18.90 & 2.02 \\
\hline APD-3 & 4.23 & 10.54 & 44.56 & 3.95 & 2.54 & 10.06 & 3.82 \\
\hline APD-4 & 4.31 & 10.67 & 46.00 & 4.13 & 1.32 & 5.47 & 6.44 \\
\hline APD-5 & 4.07 & 11.15 & 45.40 & 4.17 & 0.87 & 3.61 & 11.04 \\
\hline
\end{tabular}

Table 8.2 Width ratios for each asymmetric PEDOT:PSS Device

\begin{tabular}{|c|c|c|}
\hline Device & $\begin{array}{c}\text { Width Ratio } \\
\text { (W1/W2) }\end{array}$ & $\begin{array}{c}\text { Width Delta } \\
\text { (W1-W2) } \\
\text { (mm) }\end{array}$ \\
\hline APD-1 & 1.00 & 0 \\
\hline APD-2 & 2.09 & 5.50 \\
\hline APD-3 & 4.15 & 8.00 \\
\hline APD-4 & 8.06 & 9.35 \\
\hline APD-5 & 12.88 & 10.28 \\
\hline
\end{tabular}

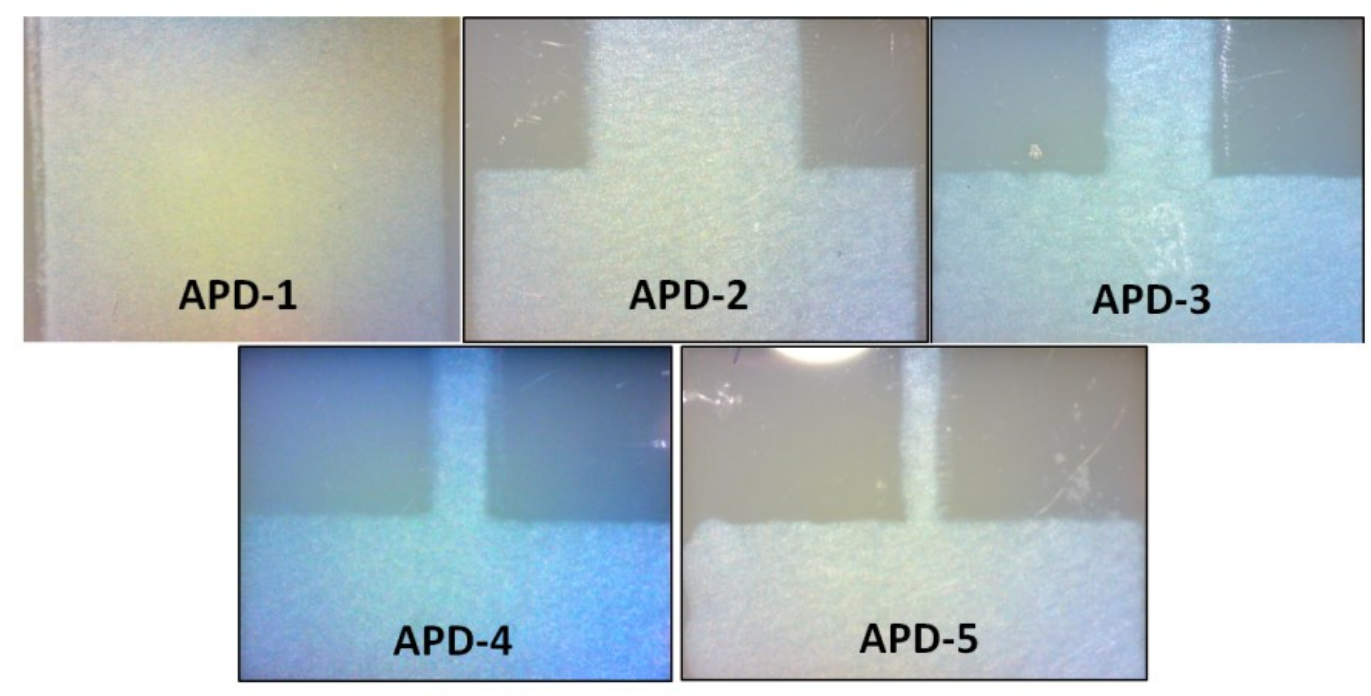

Figure 8.1 Asymmetric PEDOT:PSS devices with varying width ratios 


\subsection{Experimental Setup and Explanation}

The APDs were tested using a probing station equipped with a microscope, two gold probes, a signal generator, an oscilloscope, and data acquisition software. The probes were placed on the silver contacts of the polymer device. The positive lead of the signal generator was connected to one of the gold probes and the ground lead was connected to a sensing resistor $(\sim 10-20 \mathrm{k} \Omega$ depending on the initial polymer resistance) which was connected to the other gold probe. The oscilloscope probes were placed on the circuit to measure the voltage across the sensing resistor and the signal generator voltage, which were both recorded by the data acquisition software. Before a device was tested, a few drops of a $1.0 \mathrm{M}($ moles/ $\mathrm{L}) \mathrm{LiClO}_{4}$ electrolyte were applied to the polymer in such a way that both the narrow-width and broad-width regions were approximately covered by roughly equal amounts. The signal generator was set to output a sinusoid waveform with zero voltage offset. The frequencies used ranged from $100 \mathrm{mHz}$ to $4.0 \mathrm{~Hz}$ and the signal generator voltages were given as peak-to-peak amplitudes that ranged from $3 \mathrm{~V}$ to $8 \mathrm{~V}$.

Figure 8.2 shows a diagram for the connections mentioned above.

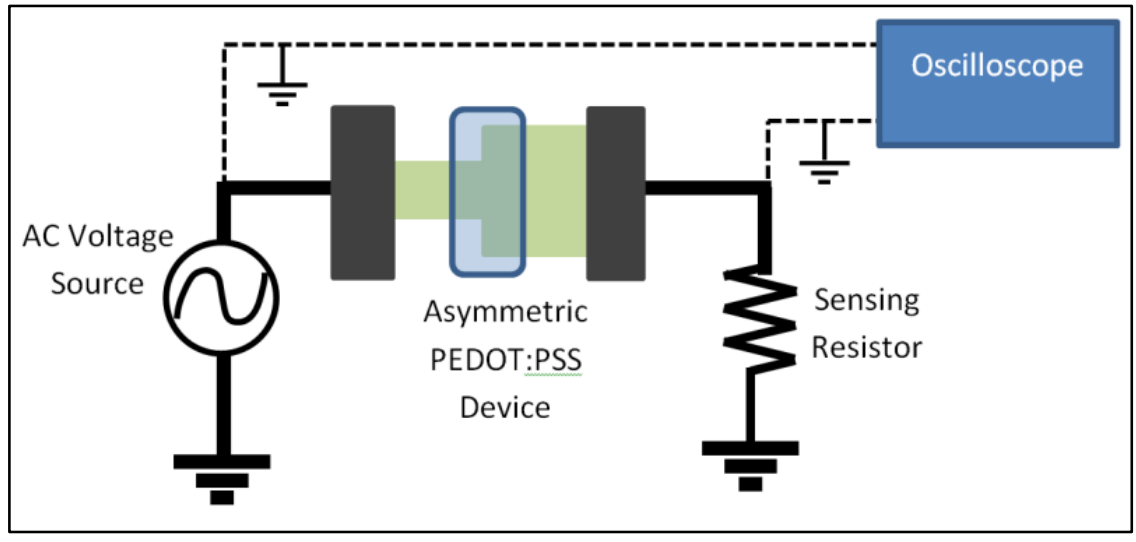

Figure 8.2 Circuit diagram used to test the asymmetric PEDOT:PSS devices 


\subsection{Results}

The electrical response of the APDs depended on the voltage amplitude and frequency used for the input voltage waveform. Since the device labelled as APD-1 was symmetric its electrical response was independent of any geometrical effect, and therefore it was chosen to illustrate the voltage amplitude and frequency dependencies.

\subsubsection{Voltage Amplitude Dependence}

The static or quasistatic behaviour of a PEDOT:PSS device revealed that its resistance increases only when a voltage threshold of about $1.5 \mathrm{~V}$ to $1.0 \mathrm{~V}$ is reached. In the case of very low frequencies $(\mathrm{mHz})$, the device functioned as a simple linear resistor below the threshold voltage. Above this threshold, its resistance began to increase and as a result it attenuated the circuit current. Figure 8.3 shows the current vs. voltage (I-V) curves for device APD-1 at a frequency of $100 \mathrm{mHz}$ and for a range of source voltages. This Figure shows a linear region between $\pm 1.5 \mathrm{~V}$ and a saturation region beyond these threshold voltage magnitudes. As a reminder, the source voltages do not correspond to the voltage across the APDs in any of the following plots unless otherwise stated. 


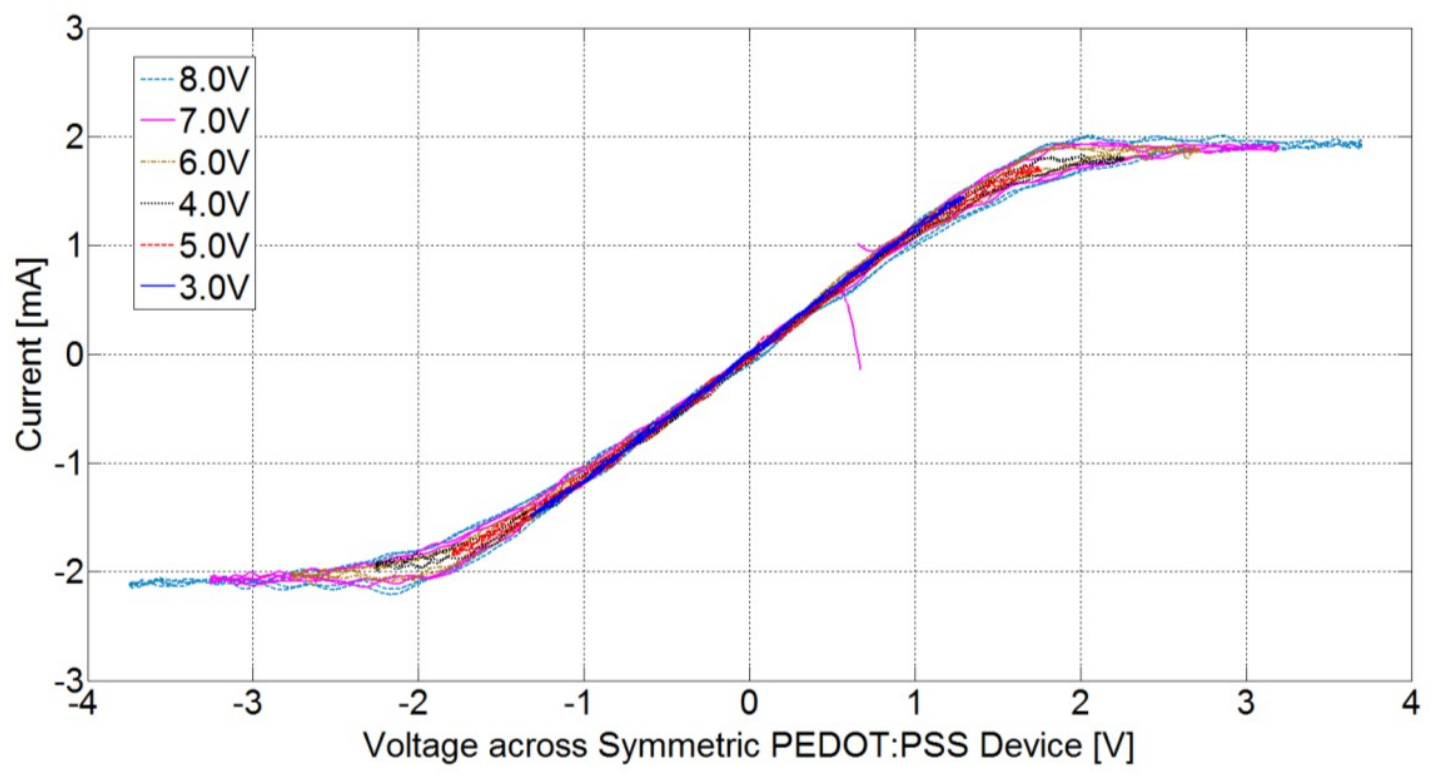

Figure 8.3 Voltage dependence on the I-V curve of a symmetric device at $100 \mathrm{mHz}$

At mid-range frequencies, the time it took for the $\mathrm{Li}^{+}$ions to intercalate into the polymer and increase its resistance became comparable to the period of the voltage waveform. Therefore, the I-V curve did not show the previously observed smooth transition into the current saturation. Instead, there was an "over-shoot" in the I-V curve which was due to the fact that the resistance cannot change as fast as the applied voltage is varying since the speed of ion intercalation is limited by the ion mobility. Figure 8.4 shows the I-V curves for APD-1, at a frequency of $1.0 \mathrm{~Hz}$ and source voltages from $3 \mathrm{~V}$ to $8 \mathrm{~V}$. One can see how the "over-shoot" in the I-V curve develops and becomes more pronounced as the curves enter the saturation region beyond the threshold voltage. 


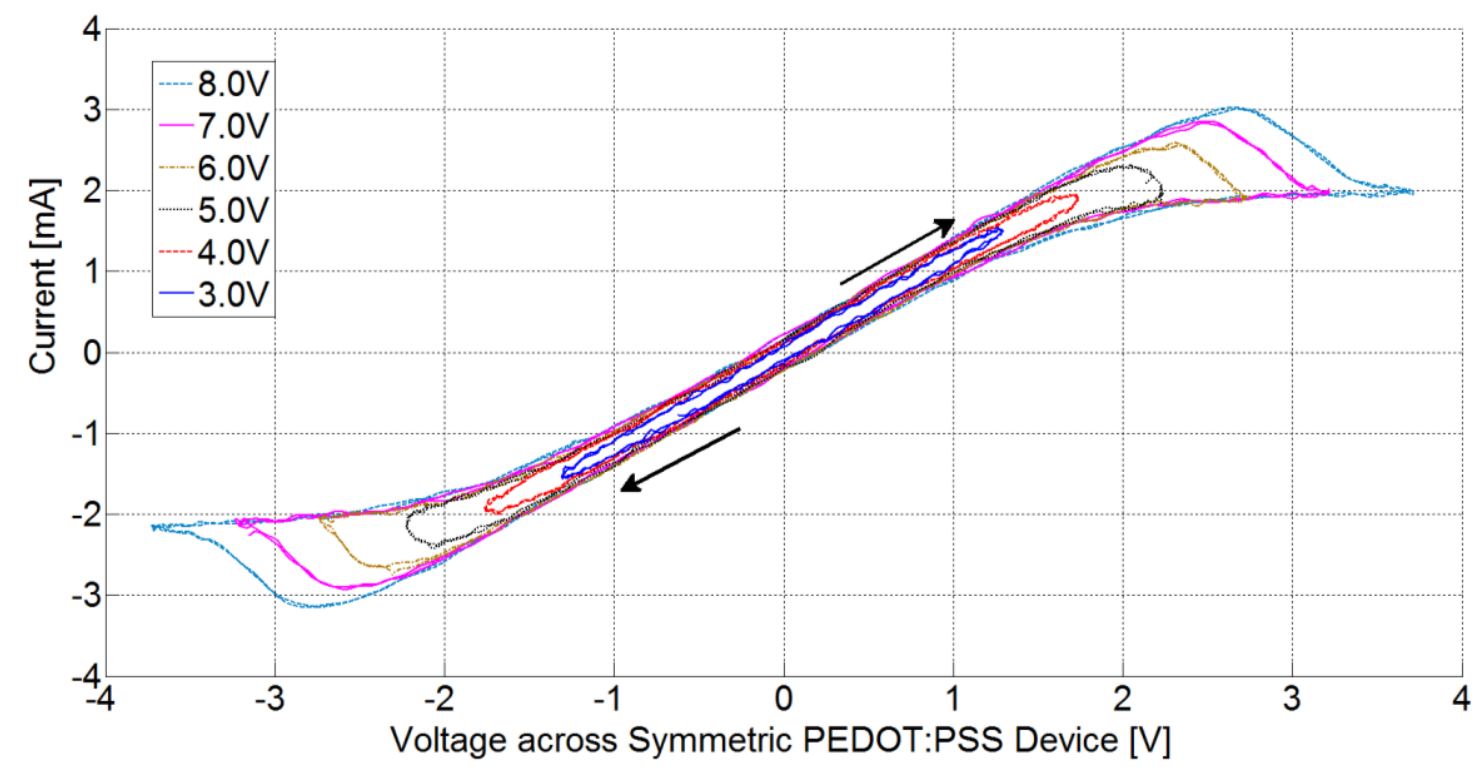

Figure 8.4 Voltage dependence on the I-V curve of a symmetric device at $1.0 \mathrm{~Hz}$

\subsubsection{Frequency Dependence}

Higher frequencies completely inhibited any resistance change due to the quick change in applied voltage and the inability of $\mathrm{Li}^{+}$ions to respond to the voltage and intercalate into the polymer. The transition in an I-V curve from having a saturation region to becoming entirely linear is illustrated in Figure 8.5. In this Figure, the IV curves correspond to APD-1 at a constant source voltage of $7 \mathrm{~V}$ and for 5 different frequencies. The reason why the I-V curve for the $4.0 \mathrm{~Hz}$ case resembled an "open-loop" instead of a straight line is because of charging and discharging effects caused by the double layer capacitance that develops at the polymer-electrolyte interface, which becomes significant at higher frequencies. This also applies to Figure 8.4 where one can see a slight loop shape in the I-V curves. 


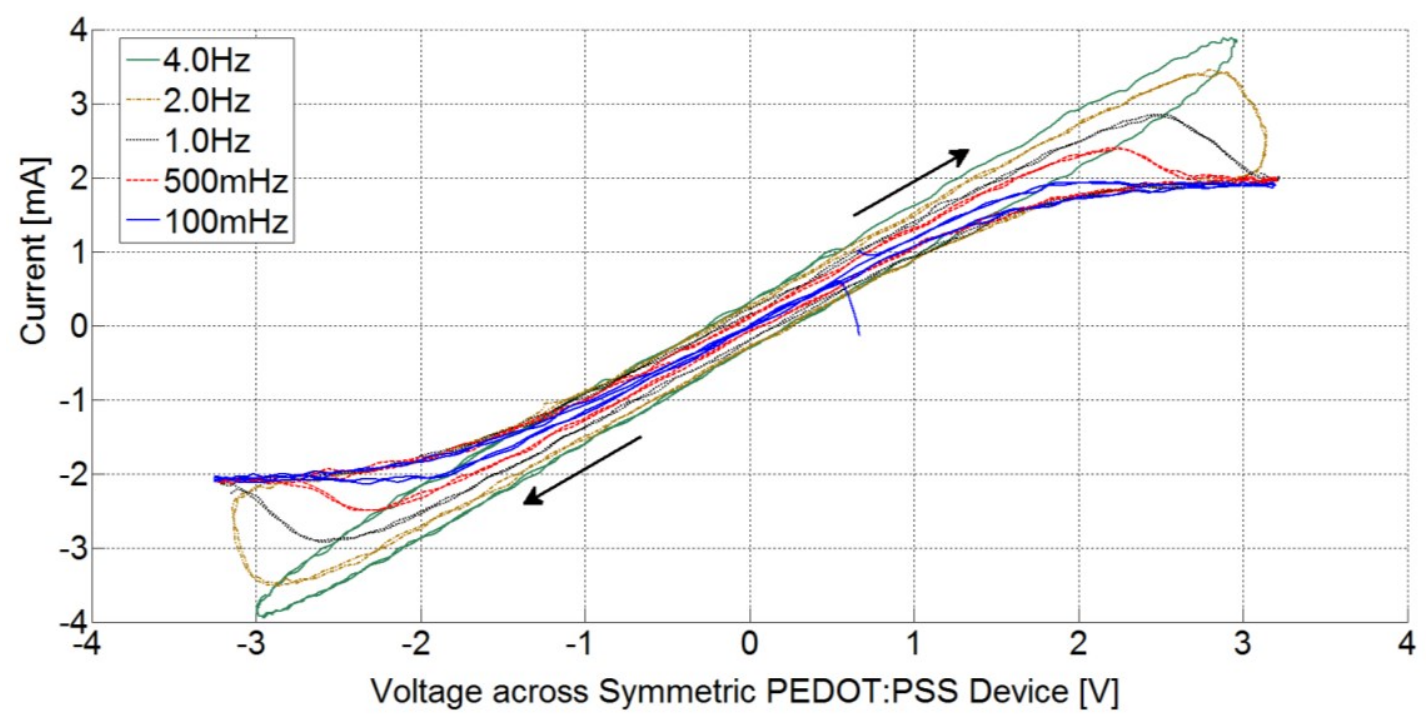

Figure 8.5 Frequency dependence on I-V curve for a symmetric device for a source voltage of 7V

\subsubsection{Geometry Dependence}

When the symmetry is broken in the device a second effect appeared in which one end of the I-V curve reached saturation at a lower threshold voltage than the other end of the curve. This occurs because the narrow-width side had a higher resistance than the broad-width side, and therefore there was a higher voltage drop across the narrow-width side than on the broad-width side. Figure 8.6 shows the electric voltage distribution throughout a symmetric and asymmetric PEDOT:PSS device obtained from a Multiphysics COMSOL simulation. Figure 8.7 shows the corresponding simulated electric field through a horizontal cross sectional path (shown by the horizontal black dotted line on Figure 8.6) of the PEDOT:PSS thin film for the symmetric and asymmetric devices. 


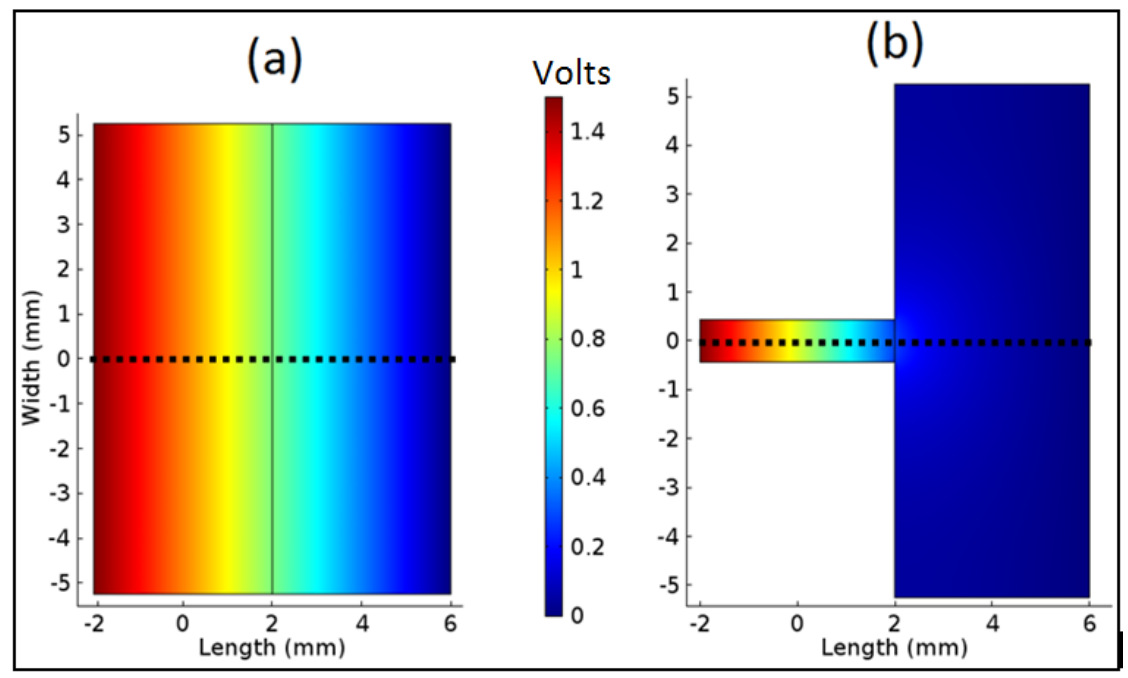

Figure 8.6 Electric potential distribution for (a) a symmetric PEDOT:PSS device [APD-1] and (b) asymmetric PEDOT:PSS device [APD-5]. Colour bar is given in units of volts

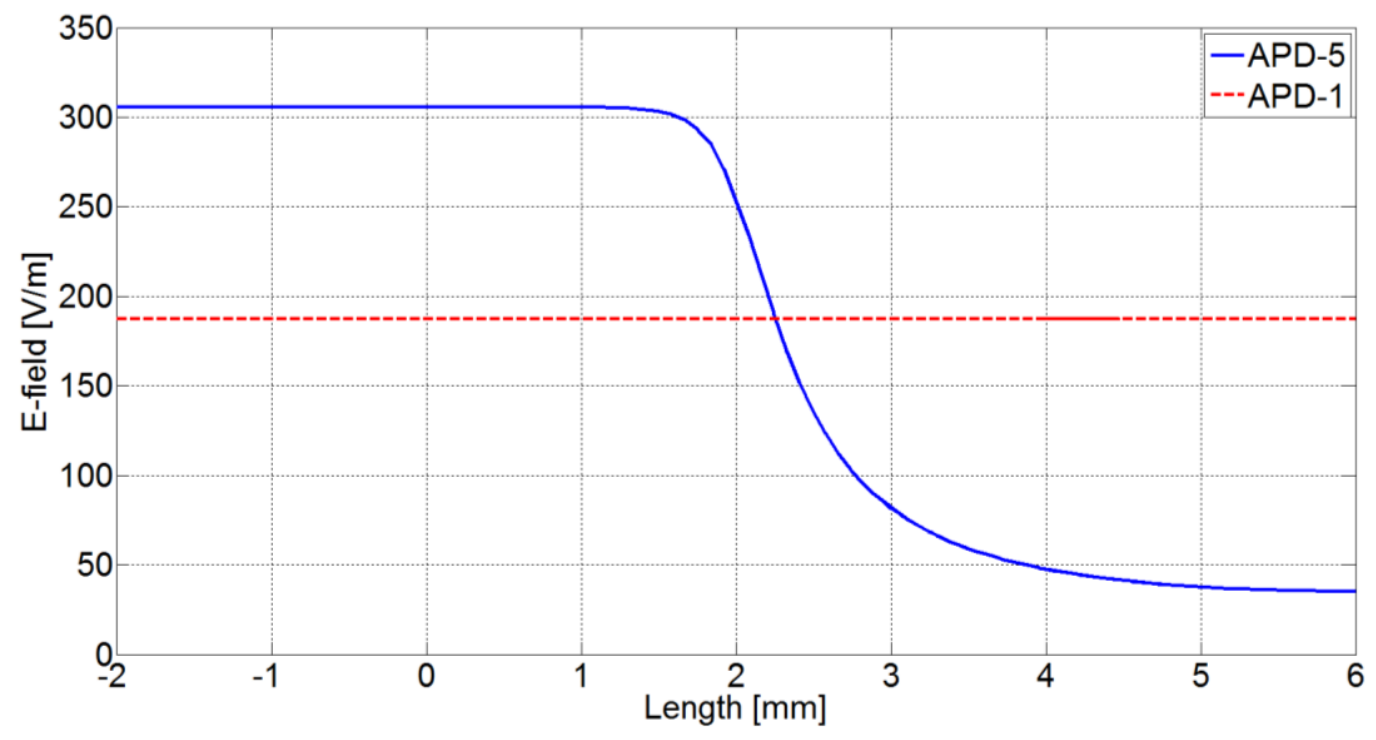

Figure 8.7 Electric Field through cross sectional path for a symmetric PEDOT:PSS device [APD-1] and an asymmetric PEDOT:PSS device [APD-5]

Both Figures 8.6 and 8.7 clearly indicate that the asymmetry in the polymer device redistributed the voltage across the polymer and it resulted in a higher electric field on the narrow-width side as compared to the broad-width side. In other words, the gradient of 
the voltage was higher on the narrow-width side and the $\mathrm{Li}^{+}$ions felt a stronger force to migrate towards the edge of the electrolyte and intercalate into the polymer on that side. As a result, the voltage threshold for the narrow-width side became lower than for the broad-width side. Hence the key factor is that the width asymmetry in the device created an asymmetry in the distribution of voltage across the device. Figure 8.8 shows the experimental I-V curves for each device, including the symmetric device (APD-1), where the applied voltage on the PEDOT:PSS device was $3 \mathrm{~V}$ (peak-peak) at a frequency of 40mHz. The inset in Figure 8.8 shows the on/off ratios for each device calculated by the ratio between the maximum resistances of the device at the ends of the I-V curves $( \pm 1.5 \mathrm{~V})$.

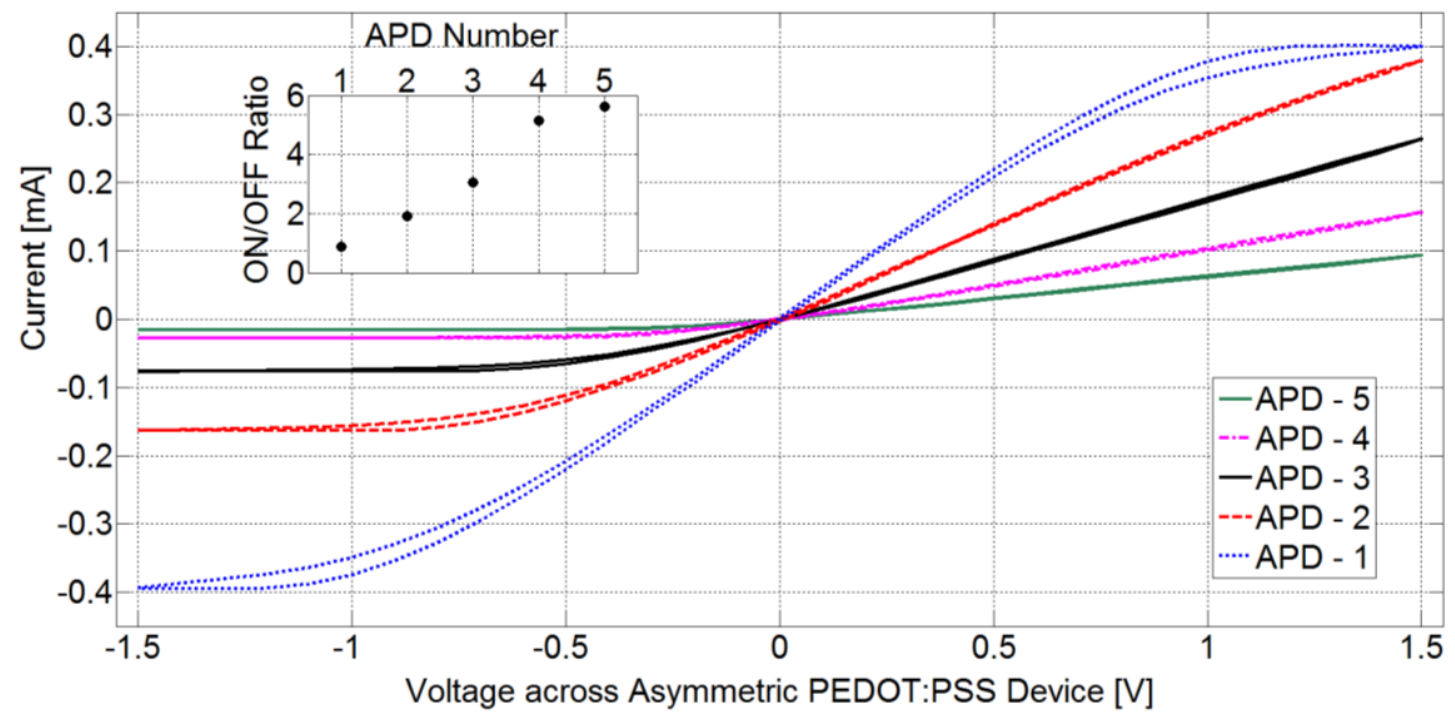

Figure 8.8 I-V curves for 4 asymmetry and 1 symmetric device for a source range of $\pm 1.5 \mathrm{~V}$ and a frequency of $40 \mathrm{mHz}$

\subsubsection{Self-Oscillatory Behaviour}

Several unexpected oscillations were observed in the measured voltage across the sensing resistor that had a higher oscillating frequency than the input AC voltage signal. 
This phenomenon is the result of an oscillating (a few Hz) change in the resistance of the PEDOT:PSS film, which resulted in the oscillation of the current through the circuit. This behaviour was observed for all devices, with the exception of APD-1, and the oscillations only appeared when the input voltage had a frequency of $100 \mathrm{mHz}$. The voltage amplitude at which the oscillations appeared was lower for APDs with a high width ratio and vice versa. Figure 8.9 shows three cases where oscillations in the current waveform were observed for APD-3 at 100mHz.

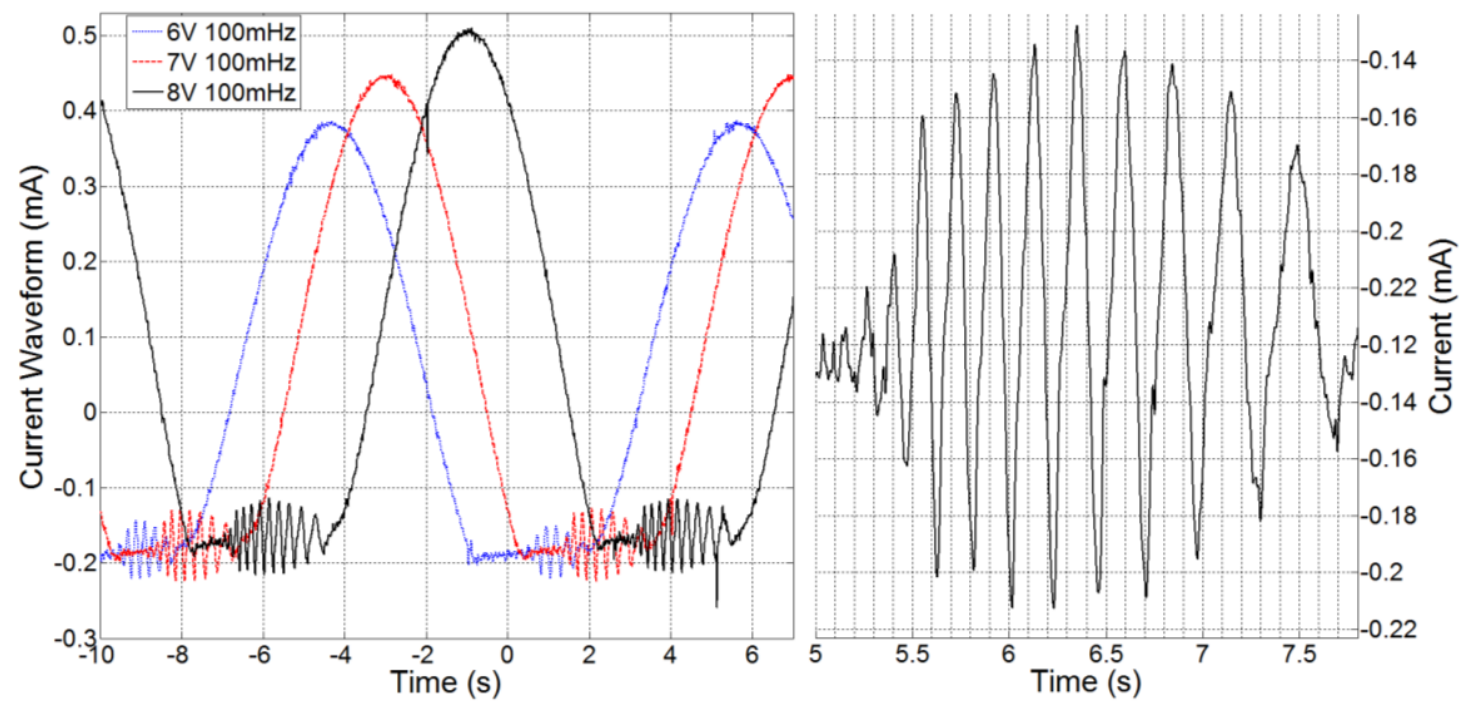

Figure 8.9 (left) Anomalous oscillatory behaviour in the electrical response of APD-3 at 100mHz. (right) close up of oscillations for $8 \mathrm{~V}$ and $100 \mathrm{mHz}$.

Figure 8.9 (right) shows that the oscillations occurred at about $2.5 \mathrm{~Hz}$ or a period of 0.4 seconds, while Figure 8.9 (left) shows that the amplitude of these oscillations grew slightly as the applied voltage increases. The appearance of oscillation in the electrical response of conjugated polymer devices has been reported by only two other authors. Smerieri et al. (2008) reported the appearance of current oscillations through a polyaniline (PANI) two terminal device when a graphite reference electrode was used as 
part of their simple two-electrode polymer element. They attributed this phenomenon to charge accumulation by the graphite electrode which caused the reference potential to vary even when applying a constant voltage to the two-terminal PANI device [43]. Tu et al. (2011) also observed a self-oscillating current in a three terminal PEDOT:PSS transistor-like device. In these PEDOT:PSS devices, the change of electrical energy to chemical energy was modelled by an inductor to make an RLC equivalent circuit model that was used to simulate the current oscillations [44]. However, to date there are no studies reporting any current oscillations present in a two terminal single channel PEDOT:PSS devices without a reference or gate electrode. This self-oscillatory behaviour is one of the many interesting attributes of conjugated polymers that must be investigated in more depth.

\subsection{Conclusions}

The electrical response of the asymmetric PEDOT:PSS devices showed that the response time of the device was clearly limited by the movement of $\mathrm{Li}^{+}$ions. Therefore, a high frequency $(>2 \mathrm{~Hz})$ signal did not allow the change in the polymer conductivity to occur as fast as the applied voltage varied. There was also a clear threshold voltage below which the polymer conductivity did not show major variations. The structure geometry in asymmetric devices redistributed the voltage drop across the device, which caused the threshold voltage on the narrow-width side of the device to be lower than on the broadwidth side. Finally, an anomalous oscillatory current was observed at certain frequencies and voltage amplitudes in the case of the asymmetric devices. This has not been 
previously reported in the literature for the type of PEDOT:PSS devices consider in this work. 


\section{Chapter 9}

\section{Thesis Conclusion}

\subsection{Summary}

This work presented the experimental results for the characterization of the main components of a PEDOT:PSS electrolytic device. In the case of the lithium perchlorate in propylene carbonate $\left(\mathrm{LiClO}_{4}-\mathrm{PC}\right)$ electrolytes, a series of PGSE NMR experiments were performed to establish the relationship between the diffusion coefficient and concentration of $\mathrm{Li}^{+}$ions. These two parameters were found to have an inverse linear relationship. The electrolyte conductivity was then calculated based on the data obtained for the diffusion coefficients of $\mathrm{Li}^{+}$ions for different concentrations.

In order to characterize the PEDOT:PSS polymer, the conductivity of the polymer in its doped state needed to be measured. This was accomplished by fabricating and measuring the conductivity of thin film PEDOT:PSS structures with varying length and width. An average conductivity of $975 \mathrm{~S} / \mathrm{m}$ was determined to be appropriate for these devices. The next step in the polymer characterization required a measurement of the polymer conductivity during the polymer dedoping process by means of a controlled redox reaction. This was accomplished by performing an in situ spectroelectrochemical experiment in which the polymer was dedoped due to an applied voltage and the polymer

conductivity and spectral attenuation were measured quasi-statically by allowing the polymer to reach equilibrium before taking the measurements. The results showed a 
sigmoidal shape for the relationship between polymer conductivity and intercalated $\mathrm{Li}^{+}$ ion concentration, where a minimum conductivity of $0.39 \mathrm{~S} / \mathrm{m}$ was obtained for $\sim 1.2 \mathrm{mC}$ of intercalated charge. Moreover, the change in conductivity during the dedoping of the polymer was reversible with an on/off ratio of 2500 . This reversible change in the conductivity had a hysteresis-like behaviour that vanished when the overall change in conductivity was not close to the maximum change possible. Since the variable resistance of the polymer and the double layer capacitance had an associated RC time constant, it can be suggested that the existence of this hysteresis-like behaviour was likely due to a difference in the RC time constant during the discharging and charging of the electric double layer formed between the polymer and the electrolyte interface.

Furthermore, the $\mathrm{Li}^{+}$ion intercalation was studied in more detail by recording a video of the movement of a blue coloration front, associated with intercalated $\mathrm{Li}^{+}$ions, caused by an applied voltage. This video analysis showed that there was a maximum displacement of $0.2 \mathrm{~mm}$ for migrating ions into a dry polymer region and that the migration of ions through the polymer had a power-law speed-time relationship. The exponent values for this power law relationship were determined to be on average 0.715 for applied voltages between $2 \mathrm{~V}$ to $5 \mathrm{~V}$.

Since the main objectives of this thesis were to provide the necessary parameters and experiment data to enable future simulation of PEDOT:PSS electrolytic devices, a group of simple asymmetric PEDOT:PSS electrolytic devices were fabricated and their electrical response was tested. The effect that the input signal frequency, voltage amplitude and structure geometry had on the electrical response of these electrolytic polymer devices was presented and discussed. The frequency response showed that 
temporal response of the devices was limited by the mobility of the $\mathrm{Li}^{+}$ions. A threshold voltage was observed in the electrical response, which needed to be reached before the polymer started to show signs of a change in its conductivity. The geometrical dependence highlighted the importance that the distribution of the electric field has on the operation of the device. Finally, an anomalous self-oscillatory behaviour was observed in the electrical response of the polymer electrolytic devices for certain applied frequencies and amplitude voltages. Although, a similar behaviour has been reported in the literature, this is the first time self-oscillation in the electrical response of a 2 terminal PEDOT:PSS is shown.

Overall, the characterization results for the PEDOT:PSS polymer and $\left(\mathrm{LiClO}_{4}-\mathrm{PC}\right)$ electrolytes provided the necessary experimental data in order to develop a simulation of polymer electrolytic devices and validate its simulation results.

\subsection{Knowledge Contribution}

The most important property of PEDOT:PSS is its variable conductivity that depends on the concentration of intercalated cations into the polymer. However, the conductivity of PEDOT:PSS is usually described as a function of the applied intercalating voltage. This work provides an estimate of the functional relationship between the conductivity of the polymer of the concentration of intercalated $\mathrm{Li}^{+}$ion which is vital to any simulation that attempts to simulate the behaviour of a PEDOT:PSS-electrolytic device.

The video analysis results for the $\mathrm{Li}^{+}$ion migration in dry and wet polymer regions is the first of its kind for screen printed PEDOT:PSS. These results included the ion speed within the polymer region which can be used to estimate the ion mobility once the 
electric field is determined for these regions of polymer. Also, the description of the $\mathrm{Li}^{+}$ ions displacement in dry and wet polymer regions as a function of time will assist future simulations of polymer-electrolytic devices to reach simulation results that replicate the behaviour of these devices.

Furthermore, the electrical response of an asymmetric PEDOT:PSS electrolytic device was measured for a number of different applied AC voltages, frequencies and asymmetry which has not been reported elsewhere in the literature. These results revealed interesting and potentially useful current-voltage curves that might find an application in neuromorphic systems. In addition to this, a self-oscillatory effect was observed for a two-terminal device which has only been reported to occur for three-terminal devices. These are the main contributions to the state of knowledge on PEDOT:PSS-electrolytic systems.

\subsection{Improvements and Future Work}

A significant improvement could be made to the spectroelectrochemical experiment by performing this experiment in conjunction with an Electrochemical Quartz Crystal Microbalance (EQCM) experiment to corroborate the results obtained for the amount of intercalated $\mathrm{Li}^{+}$ion charge. Moreover, this entire spectroelectrochemical experiment could be performed on more PEDOT:PSS electrochemical transistor devices with possible variation of the polymer film volume with the aim of further validating the results shown in this work. 
The analysis of the migration of $\mathrm{Li}^{+}$ions could benefit from better video capturing equipment that would enhance the spatial and temporal resolution of the videos. This was a limitation encountered in the video analysis performed in this work.

A simulation of the polymer electrolytic devices used in this work would be the next step to pursue. Due to the nature of these polymer devices, this simulation would require a consideration of different physics, such as mass transport of species in different media, electrostatics and steady state currents through media and possibly chemical reactions. This simulation should also provide the ability to set the polymer conductivity as a function of the $\mathrm{Li}^{+}$ion concentration. Excess charge densities should be taken into account since these will allow the electrolyte to be polarized and are a key component to the functionality of the device. This simulation could be performed using Multiphysics COMSOL as a starting point. 


\section{References}

[1] Blythe, T., \& Bloor, D. "Electrical properties of polymers". United Kingdom, Cambridge: Cambridge University Press. (2005).

[2] Griffiths, D. "Intoduction to quantum mechanics". Upper Saddle River, NJ: Pearson Education, Inc. (2005).

[3] Heeger, A.J., Sariciftci, N.S., \& Namdas, E.B. "Semiconducting and metallic polymers". New York, NY: Oxford University Press Inc. (2010).

[4] Salaneck, W.R., Friend, R.H., \& Bredas, J.L. "Electronic structure of conjugated polymers: consequences of electron-lattice coupling”. Physics Reports. 319, 231-251. (1999).

[5] Taylor, J.R., Zafiratos, C.D., \& Dubson, M.A. "Modern physics for scientists and engineers”. Upper Saddle River, NJ: Prentice Hall, Inc. (2004).

[6] Kittel, C. "Introduction to solid state physics". New York, NY: John Wiley \& Sons. (1986)

[7] Elschner, A., Kirchmeyer, S., Lovenich, W., Merker, U., \& Reuter, K. "PEDOT principles and applications of an intrinsically conductive polymer”. Boca Raton, FL: CRC Press. (2011).

[8] Bredas, J.L., \& Street, G.B. "Polarons, bipolarons, and solitons in conducting polymers". Acc. Chem. Res. 18, 309-315. (1985).

[9] Newman, J., \& Thomas-Alyea, K.E. "Electrochemical systems". Hoboken, NJ: John Wiley \& Sons, Ltd. (2004).

[10] Brett, C.M.A., \& Brett, A.M.O. "Electrochemistry principles, methods, and applications”. New York, NY: Oxford University Press Inc. (1993).

[11] Bagotsky, V.S. "Fundamentals of electrochemistry". Hoboken, NJ: John Wiley \& Sons, Inc. (2006). 
[12] Xuan, X., Wang, J., Tang, J., Qu, G., \& Lu, J. “A vibrational spectroscopic study of ion solvation in lithium perchlorate/propylene carbonate electrolyte". Phys. Chem. Liq. 39, 327-342. (2001).

[13] Brehm, J.J., \& Mullin, W.J. "Introduction to the structure of matter". Hoboken, NJ: John Wiley \& Sons, Inc. (1989).

[14] Levitt, M.H. "Spin dynamics basics of nuclear magnetic resonance". Chichester, UK: John Wiley \& Sons, Ltd. (2001).

[15] Hardy, E.H. "NMR methods for the investigation of structure and transport". New York, NY: Springer-Verlag Berlin Heidelberg. (2012).

[16] Carr, H.Y., \& Purcell, E.M., "Effects of diffusion on free precession in nuclear magnetic resonance experiments". Phys Rev. 94 (3), 630-638. (1954).

[17] Holz, M. "Electrophoretic NMR”. Chem. Soc. Rev, 23, 165-174. (1994).

[18] Groenendaal, L.B., Jonas, F., Freitag, D., Pielartzik, H., \& Reynolds, J.R. "Poly(3,4ethylenedioxythiophene) and its derivatives: past, present, and future". Adv. Mater. 12 (7), 481-494. (2000).

[19] McGarry, S.P., \& Tarr, N.G. "Fabrication and modeling of screen-printed active electrolytic polymer devices". Semicond. Sci. Technol. 23, 055009. (2008).

[20] Kirchmeyer, S., \& Reuter, K. "Scientific importance, properties and growing applications of poly(3,4-ethylenedioxythiophene)". J. Mater. Chem. 15, 2077-2088. (2005).

[21] Andersson, P., Nilsson, D., Svensson, P., Chen, M., Malmstrom, A., Remonen, T., et al. "Active matrix displays based on all-organic electrochemical smart pixels printed on paper". Adv. Mater. 14 (20), 1460-1464. (2002).

[22] Ding, Y., Invernale, M.A., \& Sotzing, G.A. "Conductivity trends of PEDOT-PSS impregnated fabric and the effect of conductivity on electrochromic textile". Applied Materials \& Interfaces. 2 (6), 1588-1593. (2010). 
[23] Nilsson, D., Robinson, N., Berggren, M., \& Forchheimer, R. "Electrochemical logic circuits". Adv. Mater. 17 (3), 353-358. (2005).

[24] Chason, M., Brazis, P.W., Zhang, J., Kalyanasundaram, K., \& Gamota, D.R. "Printed organic semiconducting devices". Proceedings of the IEEE. 93 (7), 1348-1356. (2005).

[25] Timpanaro, S., Kemerink, M., Touwslager, F.J., De Kok, M.M., \& Schrader, S. "Morphology and conductivity of PEDOT/PSS films studied by scanning-tunneling microscopy”. Chem. Phys. Lett. 394, 339-343. (2004).

[26] Nardes, A.M., Kemerink, M., \& Janssen, R.A.J. “Anisotropic hopping conduction in spin-coated PEDOT:PSS thin films”. Phys Rev B. 76, 085208. (2007).

[27] Stocker, T., Kohler, A., \& Moos, R. "Why does the electrical conductivity in PEDOT:PSS decrease with PSS content? A study combining thermoelectric measurements with impedance spectroscopy”. J.Polymer. Sci.B. Polymer Physics. 50, 976-983. (2012).

[28] Nardes, A.M., Kemerink, M., Janssen, R.A.J., Bastiaansen, J.A.M., Kiggen, N.M.M., Langeveld, B.M.W. et al. "Microscopic understanding of the anisotropic conductivity of PEDOT:PSS thin films". Adv. Mater. 19, 1196-1200. (2007).

[29] Aleshin, A.N., Williams, S.R., \& Heeger, A.J. “Transport properties of poly(3,4ethylenedioxythiophene)/poly(4-styrenesulfonate)". Synthetic Metals. 94, 173-177. (1998).

[30] Torras, J., Casanovas, J., \& Aleman, C. "Reviewing extrapolation procedures of the electronic properties on the $\pi$-conjugated polymer limit”. J. Phys. Chem. 116, 75717583. (2012)

[31] Berzina, T., Erokhina, S., Camorani, P., Konovalov, O., Erokhin, V., \& Fontana, M.P. "Electrochemical control of the conductivity in an organic memristor: a time-resolved $\mathrm{x}$-ray fluorescence study of ionic drift as a function of the applied voltage". Applied Materials and Interfaces. 1 (10), 2115-2118. (2009). 
[32] Chiu, W.W., Travas-Sejdic, J., Cooney, R.P., \& Bowmaker, G.A. "Spectroscopic and conductivity studies of doping in chemically synthesized poly $(3,4-$ ethylenedioxythiophene)". Synthetic Metals. 155, 80-88. (2005).

[33] Syritski, V., Idla, K., \& Opik, A. "Synthesis and redox behavior of PEDOT/PSS and PPy/DBS structures". Synthetic Metals. 144, 235-239. (2004).

[34] Zotti, G. "Doping-level dependence of conductivity in polypyrroles and polythiophenes”. Synthetic Metals. 97, 267-272. (1998).

[35] Morvant, M.C., \& Reynolds, J.R. "In situ conductivity studies of poly(3,4ethylenedioxythiophene)". Synthetic Metals. 92, 57-61. (1998).

[36] Aubert, P.H., Groenendaal, L., Louwet, F., Lutsen, L., Vandersande, D., \& Zotti, G. "In situ conductivity measurements on polyethylenedioxythiophene derivatives with different counter ions". Synthetic Metals. 126, 193-198. (2002).

[37] Carlberg, J.C., \& Inganas, O. "Fast optical spectroscopy of the electrochemical doping of Poly(3,4-ethylenedioxythiophene)". J. Electrochem. Soc. 145 (11), 30103014. (1998).

[38] Patil, A.O., Heeger, A.J., \& Wudl, F. "Optical properties of conducting polymers". Chem. Rev. 88, 183-200. (1988).

[39] Gustafsson, J.C., Liedberg, B., \& Inganas, O. "In situ spectroscopic investigations of electrochromism and ion transport in a poly(3,4-ethylenedioxythiophene) electrode in a solid state electrochemical cell”. Solid State Ionics. 69, 145-152. (1994).

[40] Kuratani, K., Uemura, N., Senoh, H., Takeshita, H.T., \& Kiyobayashi, T. "Conductivity, viscosity and density of $\mathrm{MClO} 4(\mathrm{M}=\mathrm{Li}$ and $\mathrm{Na})$ dissolved in propylene carbonate and $\gamma$-butyrolactone at high concentrations". Journal of Power Sources. 223, 175-182. (2012).

[41] Wang, J., Chen, X., Caironi, M., Gao, F., Yang, X., \& Greenham, M.C. "Entirely solution-processed write-once-read-many-times memory devices and their operation mechanism". Organic Electronics. 12, 1271-1274. (2011). 
[42] Johansson, T., Persson, N., \& Inganas, O. "Moving redox fronts in conjugated polymers from lateral electrochemistry in polythiophenes". Journal of The Electrochemical Society. 151 (4), E119-E124. (2004).

[43] Smerieri, A., Erokhin, V., \& Fontana, M.P. "Origin of current oscillations in a polymeric electrochemically controlled element”. J. Appl. Phys. 103, 094517. (2008).

[44] Tu, D., \& Forchheimer, R. "Self-oscillation in electrochemical transistors: an RLC modeling approach”. Solid-State Electronics 69, 7-10. (2011). 